\title{
VALUE-ADDED COMPOSITE BIOPRODUCTS REINFORCED WITH REGIONALLY SIGNIFICANT AGRICULTURAL RESIDUES
}

by

Matthew Arthur Solle

A thesis submitted in partial fulfillment of the requirements for the degree

of

Master of Science

in

Mechanical Engineering

MONTANA STATE UNIVERSITY

Bozeman, Montana

August 2018 


\section{(C)COPYRIGHT}

by

Matthew Arthur Solle

2018

All Rights Reserved 


\section{ACKNOWLEDGEMENTS}

This work was only possible through the help of many others, including faculty, fellow students, friends, and family who have supported me the entire way:

My advisor, Dr. Cecily Ryan, who has worked with me tirelessly for the last two years, sharing her knowledge and passion for environmentally mindful engineering while teaching me how to conduct research. Her mentoring has made me a far better student, researcher, and engineer.

My fellow graduate students, Jesse Arroyo, Flynn Murray, and Renn Scott, who have helped me with research work and through feedback and discussion in lab meetings. I couldn't count the number of times I asked Jesse for advice.

My undergraduate assistants, CT Calloway and Kelsey Moorhouse, who spent hundreds of hours in lab helping me build and test samples. I could not have finished this research without their incredible work ethic and their commitment to the project.

My graduate committee members, Dr. Mac Burgess, Dr. Douglas Cairns, and Dr. Stephan Warnat, for providing their knowledge base, guiding the progress of my research, and reviewing the culmination of my efforts.

Our donors, the Montana Agriculture Experiment Station for providing the wheats and barley used in the experiment, and the Montana Hemp Company for providing the hemp used in the experiment.

Finally my family, Tim, Moira, and Aaron Solle, for supporting my journey and inspiring me with the confidence to pursue anything I dreamt of doing. 


\section{TABLE OF CONTENTS}

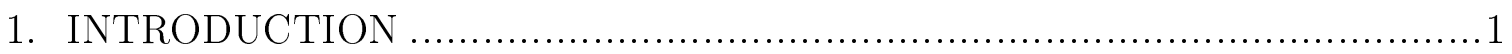

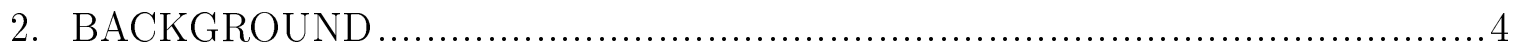

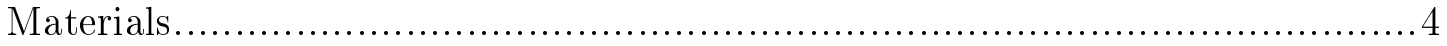

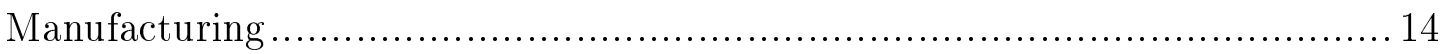

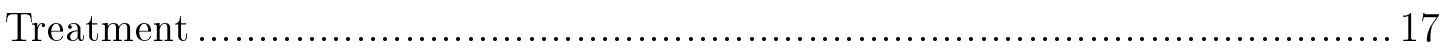

3. EVALUATION OF LOCALLY SOURCED AGRICULTURAL RESIDUE IN COMPOSITES ................................................................ 26

Contribution of Authors and Co-Authors ...................................... 26

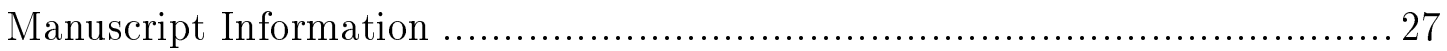

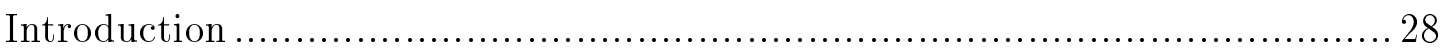

Materials and Methods .............................................................. 30

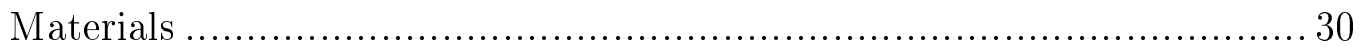

Fiber-Matrix Compatibilization ............................................... 31

Fabrication of PHBV-AF Composites ...................................... 32

Flexural Mechanical Testing............................................... 33

Rule of Mixtures Model ........................................................ 35

Statistical Design of Experiments ......................................... 35

Material Characterization ........................................................ 35

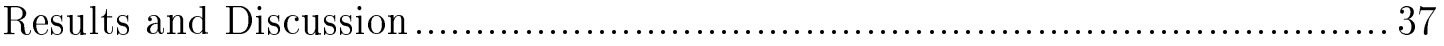

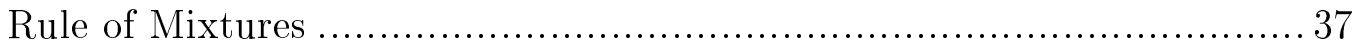

Mechanical Properties.................................................... 38

Materials Characterization ................................................. 45

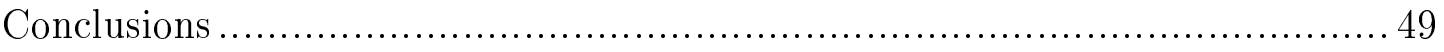

4. CONCLUSIONS AND DIRECTIONS FOR FUTURE WORK ................... 51

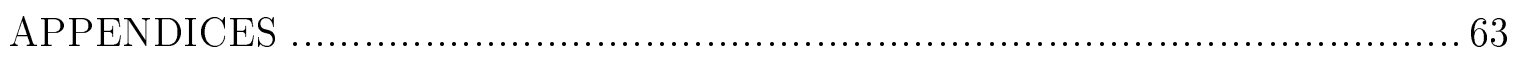

APPENDIX A : Supplementary Material ............................................. 64

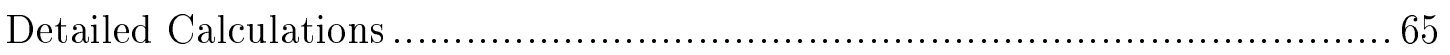

Supplementary Results and Analysis .......................................... 66

DSC Results ............................................................. 75

Fiber Fraction Analysis Results .......................................... 75

Supplementary Methods and Detailed Procedures............................... 76

Silane Coupling Agent Selection ............................................... 76

Silane Fiber Treatment Procedure ............................................ 77

PHBV Grafting Procedure .............................................. 80 


\section{TABLE OF CONTENTS - CONTINUED}

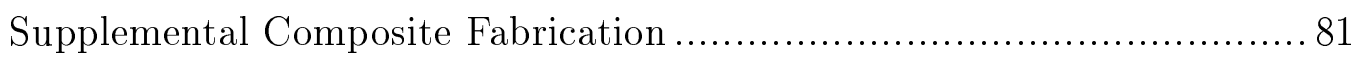

Fiber Fraction Analysis Procedure.......................................... 83

APPENDIX B : Matlab Code ..................................................... 86 


\section{LIST OF TABLES}

Table

2.1 Chemical Composition (Weight Percent) and Mechanical Properties of Common Natural Fibers............................5

2.2 Mechanical parameters of several natural fiber composites ................

2.3 Data from X Ray Diffraction Study of Untreated and Alkali Treated Bamboo Strips and Dust ............................ 20

2.4 Sample compositions and abbreviations ............................... 21

A.1 Combined Fiber Data Set ............................................... 66

A.2 Thermal properties and crystallinity of tested $20 \%$ hollow stem wheat composites .............................................. 75 


\section{LIST OF FIGURES}

Figure

Page

2.1 SEM images of (A) cellulose and (B) abaca bundle highlight the difference in size and surface roughness .................... 8

2.2 Plot of the flexural strength of laminates as a function of the fiber length utilized .................................... 9

2.3 Damages in a flax fiber after drying .................................. 15

2.4 Cellulose-I to II transition which occurs due to crystalline structure relaxation during alkaline treatment .................... 18

2.5 Banana Fiber surface after treatment, with $1 \mathrm{NaOH}$ left and $20 \mathrm{NaOH}$ right

2.6 Composite moduli and strength before creep deformation (solid bar) and after creep deformation (hatched bar)

2.7 Fully and partially biobased sandwich panel specimens

2.8 Effect of reaction time $R_{R}(\mathrm{~min})$ and total DCP concentration on the graft yield (gel\%) of CF1 and PHB (CF1, 0.5 g; PHB, 2 g; temperature $=175^{\circ} \mathrm{C}$ ).

3.1 Flexural test setup with sample and annotations.

3.2 Rule of mixtures compared to experimental values for untreated samples. Lines are the prediction of modulus using Equation 2.4 (dotted lines indicate the variation in the fiber modulus values reported in literature), and $\times$ 's are mean experimental values for untreated fiber samples.

3.3 Chord modulus of all fibers for each weight percentage, including treatments. Magenta is neat PHBV, teal is $g$ PHBV, black is untreated, blue is silane, green is grafted.

3.4 Ultimate flexural strength of all fibers by fiber, including treatments. Magenta is neat PHBV, teal is $g$ PHBV, black is untreated, blue is silane, green is grafted. 
vii

\section{LIST OF FIGURES - CONTINUED}

Figure

Page

3.5 Mechanical properties of the combined fiber set for each weight percentage, including all treatment regimens. Magenta is neat $\mathrm{PHBV}$, teal is $g \mathrm{PHBV}$, black is untreated, blue is silane, green is grafted.

3.6 The change in chord modulus caused by adding fiber with different treatments. Black is untreated, blue is silane, and green is grafted.

3.7 Differential scanning calorimetry showing the melting peak of powdered poly(hydroxybutyrate- $c o$-hydroxyvalerate) (PHBV), DCP treated PHBV prior to thermal processing, and injection molded $g \mathrm{PHBV}$. 46

3.8 Differential scanning calorimetry showing the crystallization peak of $20 \%$ hollow stem wheat untreated, silane treated, weakly grafted, and strongly grafted samples.

3.9 Differential scanning calorimetry showing the melting peak of $20 \%$ hollow stem wheat untreated, silane treated, weakly grafted, and strongly grafted samples.

3.10 FESEM results for $20 \%$ hollow stem wheat samples, where (a) is untreated, (b) is silanated, (c) is a "weak grafted" sample, and (d) is a "strong grafted" sample taken at low magnification. Sub-figures (e)-(h) are higher magnification images of the same composites

A.1 Initial points of inflection used to calculate secant modulus. The green line between data2 and data3 shows the slope used.

A.2 $\mathrm{F}$ test analysis of chord modulus of each fiber and fiber fraction by treatment. Green is significant with $99 \%$ confidence or greater, while red is not a statistically significant result. 
viii

LIST OF FIGURES - CONTINUED

Figure

A.3 F test analysis of chord modulus of each treatment and fiber fraction by fiber. Green is significant with $99 \%$ confidence or greater, while red is not a statistically significant result.

A.4 F test analysis of flexural strength of each fiber and fiber fraction by treatment. Green is significant with $99 \%$ confidence or greater, while red is not a statistically significant result.

A.5 F test analysis flexural strength of each treatment and fiber fraction by fiber. Green is significant with $99 \%$ confidence or greater, while red is not a statistically significant result.

A.6 F test analysis flexural strength of each treatment and fiber fraction by fiber. Green is significant with $99 \%$ confidence or greater, while red is not a statistically significant result.

A.7 $F$ test analysis flexural strength of each treatment and fiber fraction by fiber. Green is significant with $99 \%$ confidence or greater, while red is not a statistically significant result.

A.8 $\mathrm{F}$ test analysis flexural strength of each treatment and fiber fraction by fiber. Green is significant with $99 \%$ confidence or greater, while red is not a statistically significant result.

A.9 $\mathrm{F}$ test analysis flexural strength of each treatment and fiber fraction by fiber. Green is significant with $99 \%$ confidence or greater, while red is not a statistically significant result.

A.10 Results of fiber fraction analysis. Two samples were tested for each combination of fiber and fraction, to see the relationship between fiber fraction and stiffness. 76

A.11 Structure of GPTMS. 


\section{LIST OF FIGURES - CONTINUED}

Figure

A.12 Cup configuration with natural fibers and silane droplet on dish in center. ........................................... 78

A.13 Steady leveling curve was used as an indicator of

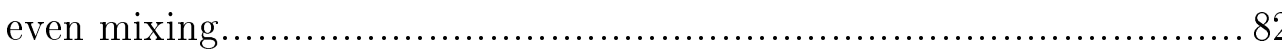




\section{ABSTRACT}

Biopolymers, such as polyhydroxybutyrate-co-hydroxyvalerate (PHBV), combined with natural fiber into biocomposites have potential as sustainable alternatives to traditional plastics and composites for which recycling is challenging. The addition of natural fibers, such as hemp, kenaf, and jute can increase the stiffness and strength of biopolymers at low weight and cost without compromising composite biodegradability. Because production of many natural fibers is limited by climate or geography, local and regional fiber sources collected as residues from agricultural crop production have potential to further reduce composite environmental impact by reducing embodied energy related to transportation and fiber cultivation. In this study four agricultural residue fibers (AF) were assessed: (i) hollow stem wheat, (ii) solid stem wheat, and (iii) barley as regionally significant food crop residues compared to (iv) hemp residue from seed and oil production as an industrially relevant control. These fibers were compounded into PHBV composites at fiber weight fractions of $0 \%, 10 \%, 20 \%$, and $30 \%$. Two fiber compatibilizing treatments were investigated for their potential to enhance the mechanical performance of AF-PHBV composites: (i) silane vapor deposited at room temperature and (ii) PHBV grafted to the fibers using reactive extrusion $(g$ PHBV). Mechanical properties including flexural modulus and ultimate flexural strength were used to evaluate the impact of fiber fraction and treatments on biocomposites. Statistical analysis from our design of experiments indicated that some combinations of fiber, weight fraction, and treatment clearly outperformed others. In particular, samples with $30 \%$ silane treated hemp had the highest modulus and high flexural strength, while $30 \% \mathrm{~g}$ PHBV hemp had high modulus and the highest strength. Among residue composites, hollow stem wheat is most comparable to hemp, with similar modulus but lower flexural strength in treated high fiber samples. Solid stem wheat and barley composites generally had lower modulus, lower strength, and less consistent mechanical properties. Increasing fiber fraction consistently increased flexural modulus. Grafted samples had inconsistent flexural strength due to deleterious effects to the $g$ PHBV matrix, as observed with scanning electron microscopy and differential scanning calorimetry. The mechanical properties of the different AF-composites occupy a similar application space, indicating potential for robust composite processing using AF. 


\section{INTRODUCTION}

Composite bioproducts, or biocomposites are composite materials produced with biobased matrix and fiber. These composites can be used in both performance demanding engineering applications (such as load bearing roof structures and bridge supports) and as low cost replacement or filler material in other applications (including furniture, shelves, and packaging material) [1,2]. They are suited to both application spaces because of a unique combination of properties. Natural fibers have low weight and cost in comparison to synthetic fibers, with comparable or even superior mechanical properties when used in composites $[3,4]$.

Biopolymers can allow for greater sustainability and reduced environmental impact over petroleum based plastics, and have surged in worldwide production, from 360,000 tonnes in 2007 to 4.2 million tonnes in 2016 and a forecasted 6.1 million tonnes in $2021[5,6]$. With 28.5 million tons of disposed and unrecycled plastic accumulating every year and 270 million tons of oil and gas dedicated to

plastic production alone, biodegradable polymers and polymers produced through non fossil fuel manufacturing processes can offset multiple environmental costs of plastic production [7]. As consumer interest in sustainable manufacturing increases, biopolymers may replace larger and larger portions of petroleum-based plastic production in the many industries which utilize them. Combining biopolymers with natural fibers can improve the mechanical properties of biopolymers and therefore their suitability for more demanding applications without compromising sustainability or biodegradability [8-10].

Fiber properties vary based on the location of production, and some fibers have 
production largely limited to specific regions, such as jute in Southeast Asia, curaua in Brazil, or sisal in South Africa [5,11]. A manufacturing plant which requires more exotic fibers like these may need to transport them over long distances or overseas, increasing the cost of the supply chain and the environmental impact of production [12]. If internationally grown crops like wheat and barley could be used effectively in natural fiber composites, they could be processed and manufactured into composites locally, reducing the monetary and ecological cost of building biocomposites [13]. Furthermore, existing fields used for agricultural crops could provide a source of fiber residue after the initial crop has been harvested, balancing the economic and environmental value of composite manufacture with the benefits of leaving this agricultural residue in place, or using it in alternate applications, such as animal bedding $[14,15]$.

The following research objectives explore the potential for developing biocomposite materials from regionally relevant agricultural residues:

1. Determine if agricultural residue fibers can be a mechanically competitive replacement for short, chopped industrial fibers (e.g. hemp) in biocomposites.

2. Assess if fiber treatments to compatibilize the fiber/matrix interface can improve the mechanical properties of composite bioproducts made with agricultural residue fibers.

3. Evaluate optimal fiber/treatment combinations to enhance mechanical properties (flexural modulus, ultimate flexural strength) for higher performance applications such as automotive panels, aircraft parts, or building construction.

To directly compare the properties of regionally significant agricultural residue to industrial fibers, four fibers from crops grown in the western United States were 
selected for examination: (i) hollow stem wheat (Triticum aestivum 'Yellowstone'), (ii) solid stem wheat (Triticum aestivum 'Genou'), (iii) barley (Hordeum vulgare 'Hocket'), and (iv) hemp (Cannabis sativa 'Finola'). All fibers were harvested, ground, and fabricated into biocomposites with PHBV, a biodegradable polymer which can be produced through either chemical synthesis or more commonly through bacterial biosynthesis $[7,16,17]$. Composites were produced through injection molding, with fiber weight percents of $0 \%, 10 \%, 20 \%$, and $30 \%$.

The fiber/matrix interface in a composite is one of the most important factors for determining the mechanical properties of the assembled composite. A weak interface will limit the mechanical benefits provided by the fibers. Incompatibilities between hydrophilic natural fibers and hydrophobic polymer matrix result in poor interfacial adhesion, leading to decreased mechanical performance and stress transfer [18]. A common solution for mitigating these issues is chemical treatment to increase the hydrophobicity of the fibers. [19,20]. Two treatments were used in this study: (i) a silane coupling agent, (3-glycidoxypropyl)trimethoxysilane (GPTMS), selected for interfacial compatibility with PHBV and room temperature (reduced energy) processing $[21,22]$ and (ii) agricultural residue fiber (AF) grafted with PHBV (AF-gPHBV), selected to modify the fiber surface with PHBV, thereby creating a chemical bond at the interface. Untreated samples were used as a control to examine the impact of treatment on mechanical properties of interest, including flexural modulus, ultimate flexural strength (UFS), strain at UFS.

To examine the interaction of fiber type, fiber fraction, and fiber treatment of PHBV biocomposites for high performance applications, an experiment was designed with an emphasis on agricultural residue and solvent free treatments. All combinations were tested for statistical significance at a $99 \%$ confidence interval with 3 degrees of freedom (for 4 fibers, 3 treatment regimens, and 4 fiber fractions). 


\section{BACKGROUND}

This review assesses the current state of processing and manufacturing of composite materials made of natural fiber and bio-derived polymers. These biocomposites have considerable potential as strong but sustainable engineering materials in a variety of applications, but are less researched than traditional engineering polymer and fiber composites. In general, natural fiber reinforced polymer composites (NFPC's) have certain advantages and disadvantages over synthetic fiber composites. These can include low weight, low cost, similar or superior physical properties, and ease of processing, at the cost of adhesion issues and a vulnerability to moisture at the fiberpolymer interface. Biodegradable alternatives to common industrial polymers pose a unique set of challenges as well, since they may interact with natural fibers and respond to chemical treatment differently.

This review will introduce common materials used in biocomposites, manufacturing techniques used in the production of biocomposites, and chemical treatments used to compatibilize natural fibers with the biopolymer matrix. Understanding the intersection of these three items is key to producing biocomposites that meet the requirements of a desired engineering application.

\section{$\underline{\text { Materials }}$}

Because the mechanical, thermal, and chemical properties of biocomposites can vary tremendously based on the fiber and polymer chosen, material selection must be done with the intended application in mind. While all natural fibers consist primarily of combinations of cellulose, hemicellulose, lignin, and wax, the presence and amounts of these ingredients can vary widely. This variation is important not only because

of the direct impact it makes on physical properties, but also because fibers with a 
different chemical makeup may respond drastically different when exposed to chemical treatments to improve stress transfer and adhesion. As can be seen in Table 2.1, natural fibers range from weak but very flexible fibers like coir to much tougher examples such as hemp and kenaf [5].

Table 2.1: Chemical Composition (Weight Percent) and Mechanical Properties of Common Natural Fibers [5, 23-26].

\begin{tabular}{|c|c|c|c|c|c|c|c|c|}
\hline Fiber & Cellulose (\%) & Hemicellulose (\%) & Lignin (\%) & Waxes (\%) & Tensile Strength (MPa) & Young's Modulus (GPa) & Elongation at Break (\%) & Density $\left(\mathrm{g} / \mathrm{cm}^{3}\right)$ \\
\hline Bagasse & $55.2 \%$ & $16.8 \%$ & $25.3 \%$ & - & $290 \mathrm{Mpa}$ & $17 \mathrm{Gpa}$ & - & $1.25 \mathrm{~g} / \mathrm{cm} \hat{3}$ \\
\hline Bamboo & $26-43 \%$ & $30 \%$ & $21-31 \%$ & - & $140-230 \mathrm{Mpa}$ & 11-17 Gpa & - & $0.6-1.1 \mathrm{~g} / \mathrm{cm} \hat{3}$ \\
\hline Flax & $71 \%$ & $18.6-20.6 \%$ & $2.2 \%$ & $1.5 \%$ & 345-1035 Мра & $27.6 \mathrm{Gpa}$ & $2.7-3.2 \%$ & $1.5 \mathrm{~g} / \mathrm{cm} \hat{3}$ \\
\hline Kenaf & $72 \%$ & $20.3 \%$ & $9 \%$ & - & $930 \mathrm{Mpa}$ & $53 \mathrm{Gpa}$ & $1.6 \%$ & - \\
\hline Jute & $61-71 \%$ & $14-20 \%$ & $12-13 \%$ & $0.5 \%$ & 393-773 Mpa & $26.5 \mathrm{Gpa}$ & $1.5-1.8 \%$ & $1.3 \mathrm{~g} / \mathrm{cm} \hat{3}$ \\
\hline Hemp & $57-77 \%$ & $9-15 \%$ & $10 \%$ & 0.8 & $690 \mathrm{Mpa}$ & $70 \mathrm{Gpa}$ & $1.6 \%$ & $1.48 \mathrm{c} / \mathrm{cm} \hat{3}$ \\
\hline Abaca & $56-63 \%$ & $20-25 \%$ & $7-9 \%$ & $3 \%$ & $400 \mathrm{Mpa}$ & $12 \mathrm{Gpa}$ & $3-10 \%$ & $1.5 \mathrm{~g} / \mathrm{cm} \hat{3}$ \\
\hline Sisal & $65 \%$ & $12 \%$ & $9.9 \%$ & $2 \%$ & 511-635 Mpa & 9.4-22 Gpa & $2.0-2.5 \%$ & $1.5 \mathrm{~g} / \mathrm{cm} \hat{3}$ \\
\hline Coir & $32-43 \%$ & $.15-.25 \%$ & $40-45 \%$ & - & $175 \mathrm{Mpa}$ & 4-6 Gpa & $30 \%$ & $1.2 \mathrm{~g} / \mathrm{cm} \hat{3}$ \\
\hline Ramie & $68.6-76.2 \%$ & $13-16 \%$ & $0.6-0.7 \%$ & $0.3 \%$ & $560 \mathrm{Mpa}$ & $24.5 \mathrm{Gpa}$ & $2.5 \%$ & $1.5 \mathrm{~g} / \mathrm{cm} \hat{3}$ \\
\hline Oil Palm & $65 \%$ & - & $29 \%$ & - & $248 \mathrm{Mpa}$ & $3.2 \mathrm{Gpa}$ & $25 \%$ & $0.7-1.55 \mathrm{~g} / \mathrm{cm} \hat{3}$ \\
\hline Curaua & $73.6 \%$ & $9.9 \%$ & $7.5 \%$ & - & $500-1150 \mathrm{Mpa}$ & $11.8 \mathrm{Gpa}$ & $3.7-4.3 \%$ & $1.5 \mathrm{~g} / \mathrm{cm} \hat{3}$ \\
\hline Barley & $31-45 \%$ & $-\%$ & $14-15 \%$ & - & - & $0.44-0.53 \mathrm{Gpa}$ & - & $0.9-1.2 \mathrm{~g} / \mathrm{cm} \hat{3}$ \\
\hline Wheat & $29-51 \%$ & $30-35 \%$ & $14-21 \%$ & - & - & $6.58 \mathrm{Gpa}$ & - & $1-1.3 \mathrm{~g} / \mathrm{cm} \hat{3}$ \\
\hline
\end{tabular}

The Centre for Advanced Materials in Universiti Tenaga Nasional, Malaysia states that the characteristics natural fibers tend to have in common include [27]:

"relatively low weight, low cost, less damage to processing equipment, good relative mechanical properties such as tensile modulus and flexural modulus, improved surface finish of molded parts composite, renewable resources, being abundant, flexibility during processing, biodegradability, and minimal health hazards."

The downsides of these fibers are almost entirely related to the adhesion between the fiber and polymer and moisture uptake of the fibers [3]. Moisture can weaken the interface, and the wide variation in chemical properties of fiber and matrix further exacerbates interfacial issues. To manage these drawbacks, various chemical 
treatments can be used, to be described later in detail. Typical synthetic polymers for use in natural fiber composites include the thermoplastics polyethylene (PE), polypropylene (PP), and polyvinylchloride (PVC), as well as the thermosets phenolic, polyester, and epoxy resins. These are the most relevant polymers to compare to when considering biodegradable options such as polyhydroxybutyrate (PHB), polylactic acid (PLA), and polyglycolic acid (PGA).

Simple Composites: Because of the variations caused by processing, treatments, and manufacturing technique, composite comparisons are more complex than a direct comparison of the basic physical properties of two polymers. Fortunately, many comparative studies have been conducted to compare one plastic with varying fibers as well as one fiber with varying plastics. One such experiment was conducted by the University of Kassel in 2010, in which PLA, PHBV, and PP were compared to each other in their pure state as well as with cellulose, abaca, and jute fibers [28]. PP makes a good basis of comparison as the most common thermoplastic for engineering/non-packaging applications. Drawbacks of the selected biopolymers include low impact strength and processing issues, therefore improving on these properties was an additional goal of the experiment.

The samples were manufactured with $30 \%$ fiber weight and $70 \%$ matrix weight. The fibers used were abaca, jute, and manmade cellulose. The PHBV was blended with $2.4 \%$ processing aids and $27.6 \%$ polybutylene adipate terephthalate (PBAT) to improve melt stability and ductility, while the PP had 5 maleic anhydride coupling agent. The materials were mixed in a single screw compounder before injection molding. Four testing methods were used to analyze the samples. For the physical parameters, tensile testing was carried out on type $1 \mathrm{~A}$ bars and A-notch impact testing was done on cutouts of $1 \mathrm{~A}$ bars. Scanning electron microscopy was used to examine the interface and morphology of the samples. Finally, fiber size was 
examined through chloroform dissolution and filtration. The parameters collected through testing included tensile modulus, tensile strength, and elongation at break. General trends identified in Table 2.2 include an increase in modulus and strength and a decrease in elongation at break with all combinations of fiber reinforced matrix. Cellulose reinforced composites tended to have the smallest increase in modulus and elongation as well as the largest increase in strength.

Table 2.2: Mechanical parameters of several natural fiber composites [28].

\begin{tabular}{c|cccc}
\hline Matrix & Fiber & Tensile Modulus, E (GPa) & Tensile strength (MPa) & Tensile elongation-at-break (\%) \\
\hline \hline \multirow{2}{*}{ PLA } & - & $3.4 \pm 0.23$ & $63.5 \pm 0.4$ & $3.3 \pm 0.5$ \\
& Man-made cellulose & $5.8 \pm 0.15$ & $92.0 \pm 4.7$ & $1.9 \pm 0.3$ \\
& Abaca & $8.0 \pm 0.34$ & $74.0 \pm 0.7$ & $1.4 \pm 0.1$ \\
& Jute & $9.6 \pm 0.36$ & $81.9 \pm 2.9$ & $1.8 \pm 0.0$ \\
PHBV/Ecoflex & - & $2.1 \pm 0.07$ & $27.3 \pm 0.3$ & $7.0 \pm 1.1$ \\
& Man-made cellulose & $4.4 \pm 0.34$ & $41.7 \pm 3.8$ & $2.3 \pm 1.0$ \\
& Abaca & $4.4 \pm 0.06$ & $28.0 \pm 1.3$ & $0.9 \pm 0.1$ \\
& Jute & $7.0 \pm 0.26$ & $35.2 \pm 1.3$ & $0.8 \pm 0.0$ \\
& - & $1.5 \pm 0.03$ & $29.2 \pm 0.4$ & $>50$ \\
& Man-made cellulose & $3.7 \pm 0.11$ & $71.6 \pm 2.7$ & $3.5 \pm 0.5$ \\
& Abaca & $4.9 \pm 0.11$ & $42.0 \pm 0.5$ & $31.7 \pm 0.2$ \\
& Jute & $5.8 \pm 0.47$ & $47.9 \pm 2.7$ & $1.4 \pm 0.1$ \\
\hline
\end{tabular}

Jute fibers had the largest decrease in elongation at break and the highest tensile modulus. Abaca fibers had the smallest increase in strength. It was believed that the small diameter of the manmade cellulose fiber contributed significantly to increased strength due to a higher aspect ratio. The smoother surface of the cellulose leads to fiber pull-out as a likely mode of failure, with an increase in fracture energy (Charpy test) and impact strength as a result. The contrast in fiber size and surface roughness of abaca and cellulose can be visually identified in Figure 2.1 [28].

To determine the critical fiber length, the minimum length for which a fiber will fully bear a load, the following series of equations was used, known as the Kelly-Tyson 

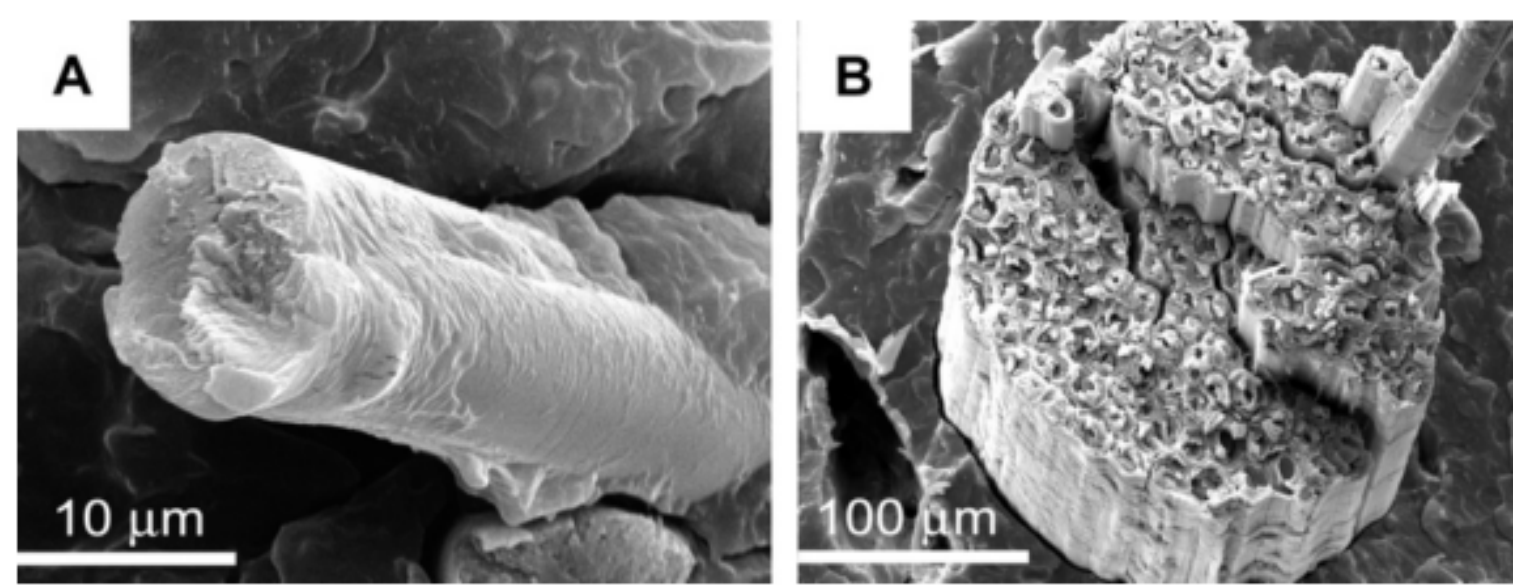

Figure 2.1: SEM images of (A) cellulose and (B) abaca bundle highlight the difference in size and surface roughness [28].

model: [28]

$$
\begin{aligned}
L_{c} & =\frac{\sigma_{f}^{\max } D_{f}}{2 \tau} \\
\tau & =\frac{\sigma_{m}^{\max }}{\sqrt{3}}=0.58 \sigma_{m}^{\max }
\end{aligned}
$$

Where $L_{c}$ is the fiber critical length, $D_{F}$ is the fiber diameter, $\sigma_{f}^{\max }$ is the maximum fiber tensile stress, $\sigma_{m}^{\max }$ is the maximum matrix tensile stress, and $\tau$ is the interfacial shear strength of the matrix. A shorter critical fiber length is indicative of a stronger matrix and interface. This relationship is because critical fiber length is determined by the threshold at which fiber will no longer shear from the interface and the fiber itself is stressed to failure. Critical length increases with $\sigma_{f}^{\max }$,as increasing tensile force on the fiber increases the likelihood of shearing at the interface. Shearing is also more likely when the fiber diameter increases and the interface is larger. The critical fiber length is only reduced through an increase in the interfacial shear strength, a function of the tensile stress of the matrix. Other studies have shown that shorter fibers allow for greater consistency across a part and may perform better than longer 
fibers with less compact structures. In one case, a linear increase in fiber length corresponded to a linear decrease in flexural strength, as shown in Figure 2.2 [29].

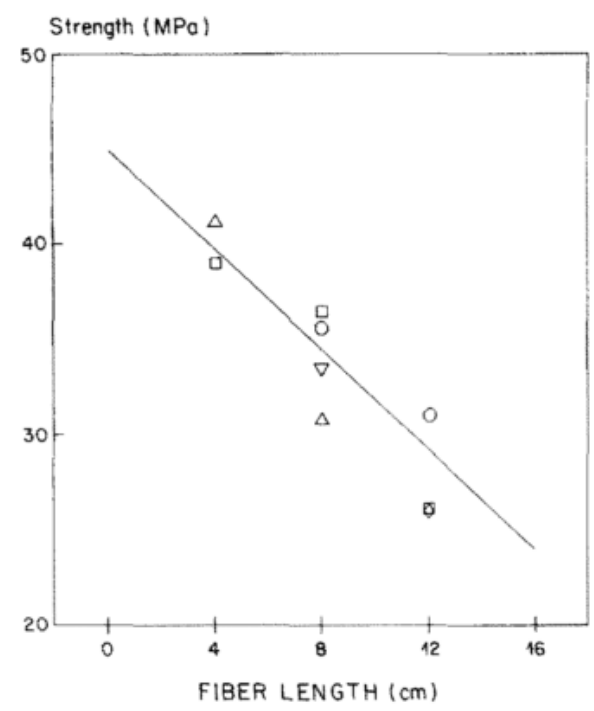

Figure 2.2: Plot of the flexural strength of laminates as a function of the fiber length utilized [29].

The Kassel study concluded that PHBV and PLA composites have potential to replace PP in many applications. However, they are held back by specific issues. PHBV underwent heavy degradation in thermal processing as well as a loss of interphase due to matrix shrinkage. The large variance in diameter of abaca caused a reduction in performance. Finally, while PLA performed well under test conditions, a low glass transition temperature of $60-65^{\circ} \mathrm{C}$ means a total loss of strength at raised temperature conditions. Suggested resolutions to this issue included thermal tempering and synthesis of compounds which have higher glass transitions.

Hybrid Composites: Some fiber/polymer combinations, such as PLA and flax, have been noted to decrease in tensile strength relative to the neat polymer alone, while PLA-jute in the same study had significantly improved tensile strength [3]. While any material selection will involve some degree of compromise, combinations 
of multiple fibers can be used to mitigate the disadvantages of single fiber composites and fine tune the product closer to the desired result. A study of hybrid fiber composites was done by the Graduate School of Science and Technology at Tokushima University in 2016 [4]. A set of three different "hybrid green composites" were made of alternating layers of kenaf (K), bamboo (B), and coir (C), within a PLA matrix. The layer compositions were KCCK, BCCB, and KBCCBK, all with the same 60/40 fiber/matrix weight percent composition. The hybrid composites were manufactured by hot press at $183^{\circ} \mathrm{C}$ and $10 \mathrm{MPa}$ for 5 minutes, with final sample dimensions $100 \times 10 \times 2 \mathrm{~mm}^{3}$. The rule of mixtures was used as a prediction of Young's modulus and laminate stress:

$$
E_{c}=E_{f} V_{f}+E_{m}\left(1-V_{f}\right)
$$

$E$ is the Young's modulus, with subscript $c$ for composite, $f$ for fiber, and $m$ for matrix. $V_{f}$ is the fiber volume fraction. To adapt this basic equation to hybrid composites, terms had to be added for each fiber type, resulting in a hybrid Young's modulus:

$$
E_{H}=E_{c} V_{f c}+E_{b} V_{f b}+E_{k} V_{f k}+E_{m}\left(1-V_{f c}-V_{f b}-V_{f k}\right)
$$

and a hybrid laminate stress:

$$
\sigma_{H}=\sigma_{c} V_{f c}+\sigma_{b} V_{f b}+\sigma_{k} V_{f k}+\sigma_{m}\left(1-V_{f c}-V_{f b}-V_{f k}\right)
$$

Where $\sigma$ is the material stress and subscripts denote the fiber type ( $c$ for coir, $b$ for bamboo, and $k$ for kenaf). This estimation relies on a set of assumptions which represents the most ideal composite build, including even fiber distribution, 
perfect fiber-matrix adhesion, and load parallel to all of the fibers. Because of these assumptions, experimentally determined values will often be lower, as adhesion in particular is a weakness of biocomposites. Rule of mixtures does not adequately capture the complex interactions between fiber and matrix and only makes for an approximation of fiber performance.

All three composites had a much higher tensile strength and modulus in comparison to neat PLA. However, only the KCCK composite had an experimental value close to the predicted modulus. This variation was attributed to impurities in the bamboo fiber which reduced interfacial adhesion, because the untreated bamboo had a chemical compound on its surface which interfered with bonding.

Since reduced environmental impact, sustainability, and biodegradability are some of the key features of biocomposites, materials selection would not be complete without examining the costs of collecting the materials. Fortunately, natural fibers are cheap to produce and often extractable from a crop being used for another purpose, such as pineapple or banana fibers which can be collected and separated during the food harvesting process.

Other fibers, such as curaua and hemp, have been produced for thousands of years already for use in spun fiber ropes, nets, and other items because of their high strength and ease of production. Co-opting an existing fiber production source without drastically impacting the main product is a good way to lower the cost and environmental footprint (particularly water usage).

Alternative fiber sources: In addition to using common industrial fibers, commercially produced plants that are not typically used for fiber have also been examined. Wheat is one of the most commonly produced crops in the world, so wheat fiber composites would enjoy the advantage of easy availability without requiring any additional commitment of land or water resources. Surprisingly, the 
research on wheat fiber biocomposites is scarce relative to most natural fibers. A study in 2015 combined wheat straw with plasticized PHBV to make food packaging with lower vapor permeability for respiring products like fruits and vegetables [30]. The plasticizers were considered necessary to counteract the brittle characteristics caused by the addition of fiber. The modulus was found to increase considerably, but at the cost of a reduced elongation and stress at break. Interfacial adhesion issues were considered the primary cause of decreased mechanical performance. A lack of fiber treatment may have contributed to this issue, in addition to PEG-400 plasticizer, which is hydrophilic and may have increased the hydrophilic/hydrophobic divide between polymer and fiber. Further testing of wheat fiber composites with an emphasis on improving the interface with treatment would be a useful subject for further work.

Perhaps the easiest fiber sources to utilize and a subject of increasing interest are the fibers of invasive plants. Invasive species tend to be widespread, grow rapidly, and thrive in a variety of conditions, to the detriment of native plants. In a multidepartment collaboration at Ohio State University, common reed, water celery, and reed canary grass were cited as examples of species which "reduce biodiversity, offer little benefit to wildlife, and choke waterways" [31]. If an industrial use could be found for these plants, then harvest of the plants for fiber would serve as an incentive to collect and control their growth. The invasive plant fibers were mixed with PHBV at fiber weight percentages of $2 \%, 5 \%$, and $10 \%$ in a twin-screw extruder, then injection molded and tensile tested. It was found that common reed and reed canary grass composites did have statistically significant increases in tensile modulus with the addition of fiber, indicating that the potential for industrial usage of these species does exist. As with other species of fiber, failure was primarily caused by interfacial adhesion issues, which is consistently cited in publications as a weak point of natural 
fiber composites.

Production can be regionally specific. Curaua is exclusively produced in Brazil [11], so any product which required these fibers should factor in the resources required to extract the fibers and transport them for processing/manufacturing. Likewise, most hemp is produced in China and most jute is produced in India, so a combination of factors both local and international may all contribute to the life cycle impact of natural fiber composites.

Bioplastic matrix: Biodegradable polymers are produced through various methods, some less resource intensive than others. Polyhydroxyalkanoates (commonly referred to as PHAs) are a microorganism generated polyester thermoplastic. They are industrially produced by bacteria provided with a carbohydrate source, such as sugar beets, and in the case of polyhydroxybutyrate-co-hydroxyvalerate (PHBV) microorganisms can be fed with methane produced by landfills, presenting an opportunity to reuse waste products with a closed cycle (PHBV degrades and releases methane which can be used to make more PHBV [7,16,17]. It has been found that PHBV performs favorably to other common polymers such as PP, PS, PVC, PLA, and HDPE in terms of $\mathrm{CO}_{2}$ release and fossil fuel consumption, particularly when fed with biogas to further offset production impact [12]. Experiments have been successfully conducted to produce PLA through a similar one-step synthesis process using modified Escherichia coli [32]. Although this method has not been widely adopted, the authors point out the possibility of creating other polymers using this process as well, opening further opportunities for the use of renewable resources in polymer production. 


\section{Manufacturing}

Key factors which impact biocomposite performance include fiber orientation, fiber physical properties, matrix physical properties and fiber-matrix interactions. The precise mode of stress transfer may have a large role to play as well. As with more conventional composites, the ability to bear a load changes drastically with fiber orientation, a 45-degree angle change resulted in a $78 \%$ loss of tensile strength in one experiment [3]. If the mode and direction of stresses is not known, then biocomposite design may focus on improving properties of tensile strength, flexural strength, modulus, and strain behavior under a standard applied load.

Volume Fraction: In general, increasing fiber volume fraction will increase the tensile performance up to a certain point, after which the addition of fiber will negatively impact strength and modulus. One mechanism for this behavior is the combination of loose fibers into bundles which reduces the interfacial area, which regularly occurs at volume fractions exceeding $50 \%$ [3]. The fiber size and length play into this process as well. If the fiber volume is achieved through large numbers of short fibers, the potential for cracking is raised relative to the same volume of longer, thinner fibers as more fiber ends act as crack initiators [3]. No consensus seems to exist on an optimal fiber fraction for tensile or flexural properties, as each fiber/matrix combination has varying behavior, further affected by processing and manufacturing.

Drying: The importance and impact of fiber drying before manufacturing has been examined, with somewhat contradictory conclusions. Pre drying is the standard practice for use of natural fibers, both for uniformity of weight and properties and because moisture content can negatively impact the mechanical performance and biodegradation rate of the composite [5]. Moisture inside a polymer composite may be unevenly concentrated in a way which creates flaws or inconsistencies in 
strength. However, the drying process itself may do more harm than good if the temperature used is too high. One study found that drying of flax fibers degraded the microfibrils and inner structure of the fiber (as shown in Figure 2.3), and caused increased crystallinity, chain scission, and depolymerization [33]. This degradation was attributed to high temperatures of $105^{\circ} \mathrm{C}$ for 14 hours of drying. Another study marked the stages at which degradation occurs in flax fiber [34]. The study concluded that wax degrades at $120^{\circ} \mathrm{C}$, pectins decompose at $180^{\circ} \mathrm{C}$, and cellulose/hemicellulose degrade at $230^{\circ} \mathrm{C}$.
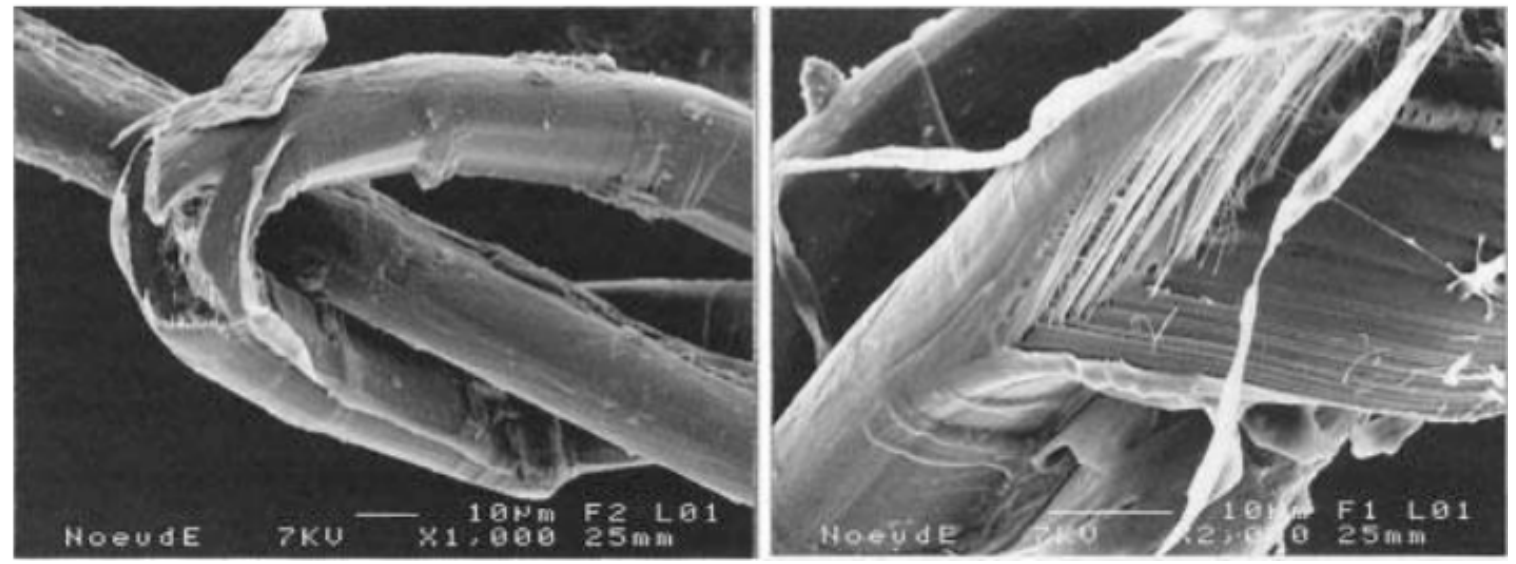

Figure 2.3: Damages in a flax fiber after drying [33]

This effect is not limited to flax, another study found decreases of $10-25 \%$ in tensile strength and 3-20\% in flexural strength for jute and hemp, with a suspected mechanism being fiber damage during the drying process [12]. More research on the tradeoffs between drying and not drying pre-manufacture, as well as the impact of drying temperature/time may be useful in the future.

Injection Molding: The ability of the user to control mechanical properties depends in large part on the manufacturing method used to produce the biocomposite. Injection molding, once optimized for the intended product, is very fast and able to handle large scales of production. Since biocomposites need to be economically viable 
to reach more mainstream usage, it makes sense that a high speed, (relatively) low cost method is common. However, the performance of injection molded parts is heavily dependent on the mixing and compounding process that precedes it. This step is where the fiber fraction is determined, and a poor compounding process may lead to flaws in the molded part as well as changes in fiber fraction across the geometry of a composite. Once the fiber and polymer have been mixed evenly, the temperature and pressure of the injection process may still cause flaws or gaps from uneven cooling, as well as an unintended fiber direction or distribution. The temperature and pressure of the injection process control the viscosity and rate of infill of the melt. A high temperature and high injection pressure lead to a rapid fill with low viscosity, which may cause fiber to flow to the outer extents of the mold cavity [35]. Poor fiber distribution will likely reduce mechanical performance.

Hot Pressing: Another manufacturing method used with biocomposites which counters some of these weaknesses is hot pressing. Hot pressing allows for more precise arrangement of fiber and matrix arrangements, including layers of multiple fibers which would normally be dispersed throughout a composite. The fiber bed is prepared in advance and then impregnated with resin, at which point any other additives are added as well [29]. Then the mix is held under high pressure and constant temperature for a certain period of time (in this article parameters were $1000 \mathrm{~kg}$ and $140^{\circ} \mathrm{C}$ for 15 minutes). The most obvious drawback of this method seems to be the increased time to produce individual composites. Prepping fiber beds, allowing time for resin infiltration, and the pressing process itself all consume time and effort that is not ideal for mass production or automation.

When it comes to fiber-matrix interactions, the primary issue seems to be the contrast between hydrophilic fibers and hydrophobic matrix, which, when combined with the presence of moisture, leads to large reductions in mechanical properties. The 
smoothness of the fiber also impacts the strength of the interface through physical interaction. These issues tend to be managed by treatments of the fiber surface.

\section{Treatment}

Many different chemical treatments have been researched and applied to improve the fiber/matrix interface. Some of these include acrylation, silanation, malleated coupling agents, dicumyl peroxide, maleic anhydride, and acetylation grafting, with the stated purpose of all treatments being to improve interfacial bonding and stress transfer [27]. Most treatments appear to have a direct and physical impact on the fiber surface.

There are many considerations when selecting a successful compatibility treatment for a given combination of fiber and polymer. However, several comparative studies have been done which yield interesting results. A combination of curaua fibers and PBAT were treated with acid hydrolysis, silane, and acetylation, then tested using DMA and tensile methods. Interestingly, acetylation treatment resulted in the highest tensile modulus and elongation at break. Another study comparing MA grafting and silane treatment on PHBV and oak wood flour composites found that silane treated composites had a higher modulus of elasticity than the untreated composite and the grafted composite [20]. Because the materials, fabrication techniques, and treatment procedures may vary greatly from study to study, mechanical comparisons of composites with different treatments must be done on a case by case basis with as few variables as possible.

Alkali Treatment: A study by Anna University, India, tested the effect of different concentrations of $\mathrm{NaOH}$ on $15 \mathrm{~mm}$ long banana fiber in composites with an epoxy matrix [18]. Alkali treatment was determined to be a cheap yet effective option. The banana fibers were submerged in different concentrations of $\mathrm{NaOH}(0.5 \%, 1 \%$, 
$2 \%, 5 \%, 10 \%, 15 \%$, and $20 \%$ by weight). After 30 minutes they were removed, rinsed, and dried in an oven. The composites were assembled via hand layup and tested with DMA from $30-140^{\circ} \mathrm{C}$ at $10^{\circ} \mathrm{C} / \mathrm{min}$ at $0.1,1$, and $10 \mathrm{~Hz}$. The specimens were analyzed before and after testing using SEM. After the first set of physical tests, specimens were submerged in water for 18 days and then tested again to measure changes in properties at saturation.

$\mathrm{NaOH}$ caused fiber swelling and changed the lattice structure of cellulose-I to cellulose-II as the crystalline structure relaxes (see Figure 2.4). This structure is more hydrophobic and was expected to improve the interfacial adhesion to different degrees based on concentration. Stronger interfacial adhesion should correspond with a direct increase in tensile strength.

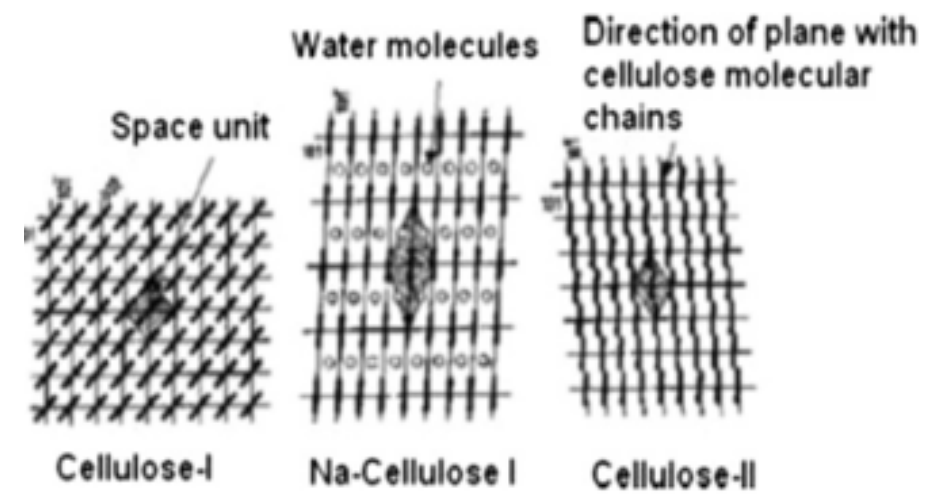

Figure 2.4: Cellulose-I to II transition which occurs due to crystalline structure relaxation during alkaline treatment [18].

It was found that the highest values of tensile strength, flexural strength, and impact strength were all achieved with $1 \% \mathrm{NaOH}$. The $1 \%$ treatment also absorbed the least moisture by reaching $6.72 \%$ at saturation after 12 days, and suffered the smallest reduction in physical properties after saturation. It was clearly the best concentration for improving the strength of the composite. Increasing percentages of $\mathrm{NaOH}$ caused larger drops in tensile, flexural, and impact strength, and increased 
water absorption. While the tensile and impact strength of the high concentration treatments were still higher than the untreated samples, the moisture absorption, flexural strength, and properties after saturation were worse than untreated fiber.

SEM revealed the cause of this problem (Figure 2.5). While $0.5 \%$ and $1 \% \mathrm{NaOH}$ caused an overall improvement in the sample properties, any higher concentrations caused degradation in the fiber itself, which caused decreased strength and created additional surface area for moisture absorption. An increase in surface roughness and peeling could be visually identified in the SEM images, particularly when comparing fiber treated with $1 \%$ concentration to $20 \%$ concentration (see Figure 2.5).
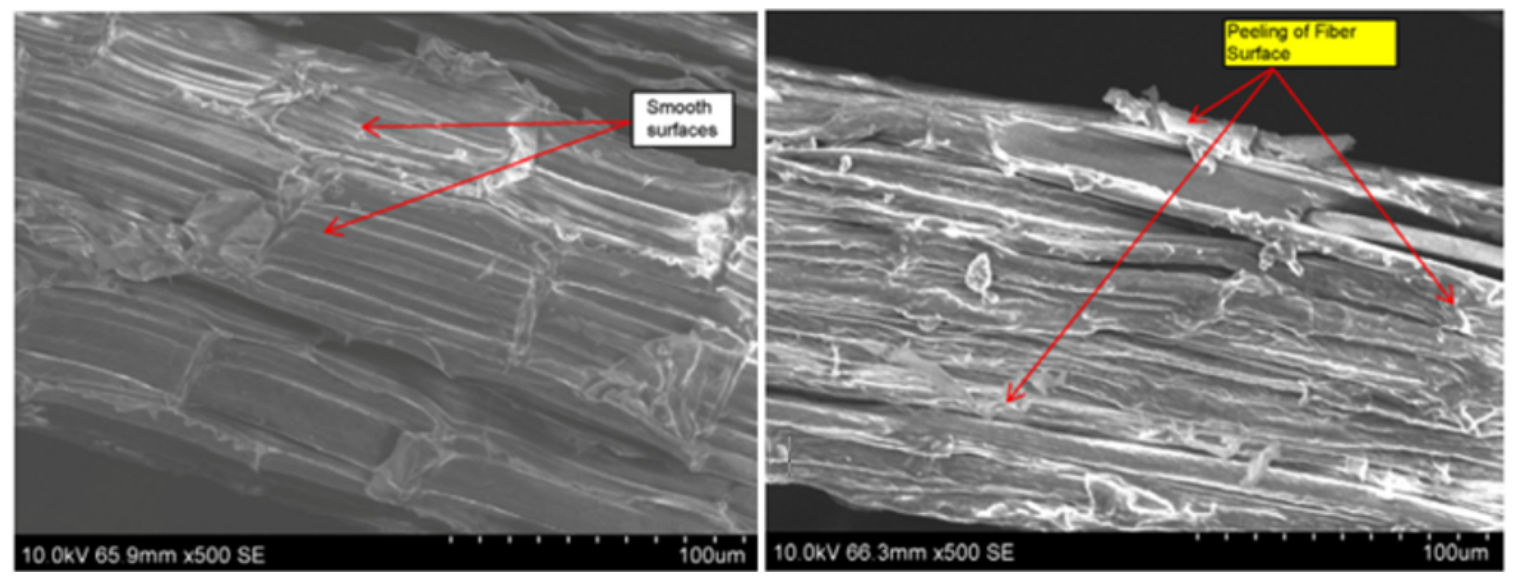

Figure 2.5: Banana Fiber surface after treatment, with $1 \mathrm{NaOH}$ left and $20 \mathrm{NaOH}$ right [18].

The diminishing returns of increasing alkali concentration for fiber pre-treatment have been noted in other studies, including a previous study on bamboo fiber in which samples subjected to $\mathrm{NaOH}$ concentration up to $15 \%$ had increasing crystallinity and transition to cellulose-II [36]. Concentrations above $15 \%$ led to reduced crystallinity and physical properties. As shown in Table 2.3, crystallinity, degree of crystallinity, and Cellulose-II content increase with smaller concentrations of alkali treatment, but drop off considerably at higher concentrations, with $50 \%$ concentration samples 
having the lowest values of crystallinity, degree of crystallinity, and Cellulose I content.

Table 2.3: Data from X Ray Diffraction Study of Untreated and Alkali Treated Bamboo Strips and Dust [36].

\begin{tabular}{|c|c|c|c|c|c|c|c|c|c|c|}
\hline \multirow[b]{2}{*}{ Sample } & \multicolumn{2}{|c|}{ Crystallinity (\%) } & \multicolumn{2}{|c|}{$\begin{array}{l}\text { Degree of } \\
\text { crystallinity }\end{array}$} & \multicolumn{2}{|c|}{ Amorphous (\%) } & \multicolumn{2}{|c|}{ Cellulose-I (\%) } & \multicolumn{2}{|c|}{ Cellulose-II (\%) } \\
\hline & Strip & Dust & Strip & Dust & Strip & Dust & Strip & Dust & Strip & Dust \\
\hline B-0 & 45.57 & 43.54 & 0.6751 & 0.667 & 54.43 & 56.46 & 45.57 & 43.54 & 0 & 0 \\
\hline B-10 & 50.1 & 45.84 & 0.6939 & 0.6762 & 49.9 & 54.16 & 35.46 & 38.23 & 14.64 & 7.6 \\
\hline B-15 & 51.48 & 44.82 & 0.6955 & 0.651 & 48.2 & 55.18 & 34.03 & 31.01 & 17.85 & 14.81 \\
\hline B-20 & 47.55 & 41.6 & 0.6846 & 0.634 & 52.55 & 58.4 & 29.71 & 8.52 & 17.84 & 33.08 \\
\hline B-50 & $17.82^{\mathrm{a}}$ & $18.51^{\mathrm{a}}$ & 0.5792 & 0.5309 & 82.18 & 81.49 & 1.94 & 1.71 & 15.88 & 16.8 \\
\hline
\end{tabular}

${ }^{\mathrm{a}} I_{\max }=I_{101}$, in all other cases $I_{\max }=I_{002}$.

The mechanisms of $\mathrm{NaOH}$ treatment were described in additional detail in a review also published by Anna University [2]. Treatment within a certain limit of concentration works to break hydrogen bonds in the cellulose, expose crystallites, and clean the surface of the fiber of lignin, wax, and oils. All of these effects contribute to the improved performance of the composite. However, at an increased concentration, excessive lignin breakdown causes fiber surface damage, as was seen in the previous study.

Silane Treatment: Another common treatment used to address the fibermatrix interface is the usage of various silane coupling agents, such as octadecyltrimethoxysilane. Like alkali treatment, one method of silane treatment begins with submerging fiber in a solution with a low concentration $(\sim 2 \%)$ of silane, mixed with 40:60 water and alcohol [11]. After 5 minutes, the fiber is removed and air dried. The silane acts as a coupling agent by creating covalent bonds which contribute to a cross linked network. This treatment limits the effect of moisture at the interface and strengthens the tensile and thermal properties of the composite.

A comprehensive examination of PHBV and wood flour (WF) composites treated with silane as well as maleic anhydride grafting was conducted by researchers at Stanford University $[10,12,19,20,37]$. In one study, creep deformation was the 
parameter of interest, as it is an important measure of durability in construction applications [12]. The samples were placed under sustained flexural loading in increasing percentages of the ultimate flexural strength. Creep deflection was measured during loading, and then strength losses due to creep were examined through a mixed experimental and theoretical approach. The sample compositions used are shown in Table 2.4.

Table 2.4: Sample compositions and abbreviations (reproduced from Miller [12]).

\begin{tabular}{c|c}
\hline Abbreviation & Composite Constituents \\
\hline P20 & Neat PHBV \\
P40 & PHBV with approximately 20\% wood flour by weight \\
M & PHBV with approximately $40 \%$ wood flour by weight \\
M20 & Maleated PHBV \\
M40 & Maleated PHBV with approximately 40\% wood flour by weight \\
S20 & PHBV with approximately 20\% silane coated wood flour by weight \\
S40 & PHBV with approximately $40 \%$ silane coated wood flour by weight \\
PP26 & Polypropylene with $26 \%$ wood flour by weight \\
PP40 & Polypropylene with $40 \%$ wood flour by weight \\
\hline
\end{tabular}

They found that untreated PHBV samples, with 0\% (P), 20\% (P20), and 40\% (P40) weight WF had a significant increase in flexural modulus between $0 \%$ and $20 \%$ WF, but no significant change between $20 \%$ and $40 \%$ (Figure 2.6). In addition, a large decrease in flexural strength occurred in the $40 \%$ WF samples. This was suspected to be the result of WF dispersing unevenly and poor fiber wettability. For specimens subjected to maleic anhydride grafting (M, M20, M40), the flexural modulus increased steadily from $0 \%$ to $40 \%$ WF. On the other hand, flexural strength for the $0 \%$ and 
$20 \%$ WF samples was roughly the same, and then suffered a drop at $40 \%$ similar to the untreated sample. One unusual behavior the author pointed out was a slight decrease in the maleated PHBV (M) flexural modulus compared to the untreated PHBV (P). The grafted samples undergo larger elastic deformation but have the same ultimate strength.
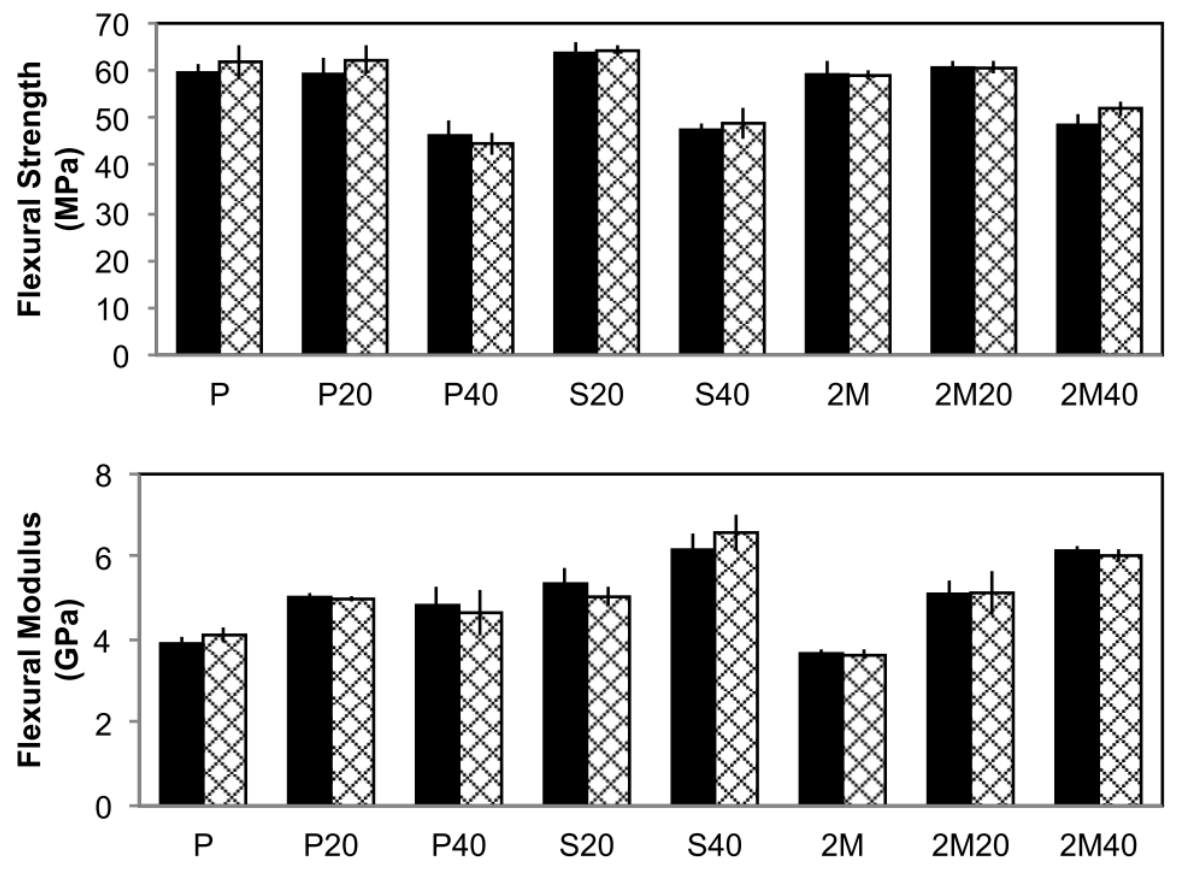

Figure 2.6: Composite moduli and strength before creep deformation (solid bar) and after creep deformation (hatched bar) [12].

The silane treated specimens (S20, S40) had the same overall behavior as the maleic anhydride specimens, with gains in modulus but a drop in strength at $40 \%$. S20 had slightly higher strength than M20, while M40 had a slightly higher strength than S40.

While none of the specimens had significantly altered properties after creep deformation, the treated composites did have slightly less strain. The tests were at a small and largely recoverable amount of stress, so drastic changes were not 
expected. Despite having similar end results, the mechanisms by which these treatments improved the biocomposite were very different. Silane promotes van der Waals forces at the interface, while maleic anhydride grafting adds a carboxylic group to the PHBV which can form a covalent bond with a hydroxyl group on the fiber.

Untreated Composites: While different fiber treatments lead to significant mechanical improvements, it is important to note that the chemicals themselves are not always as environmentally friendly as the basic materials, and may be nonbiodegradable or even toxic. To avoid making this compromise in properties for environmental drawbacks, Aaron Michel of Stanford University tested the feasibility of untreated biocomposites which rely solely on interlock and frictional forces (Figure 2.7) [38].

The application of interest was insulated sandwich panels, a structure which could be used for replacing plywood and timber structures with a mechanically and thermally similar material with a lower energy usage and closed recycling loop. Sandwich panel layering was seen as a possible way to utilize the advantages of biocomposites in a configuration which augments the material against flexural and shear deformation by increasing stiffness with a minimal impact on weight. Woven hemp fabric and jute burlap were used as reinforcements inside of PHBV face sheets, with the PHBV and fabric combined in alternating sheets by hot pressing. These sheet composites were glued with a bio based adhesive to a biofoam core. They were compared with four point bending and thermal analysis to sandwich panels made of industry standard synthetic materials, and found to perform similarly. Bio based panels had a higher stiffness but lower ductility.

Two methods of "accelerated weathering" were used to simulate the degradation of the biocomposites over time. They utilized UV radiation and temperature changes, with one method involving no moisture and the other involves a 30-minute water spray 


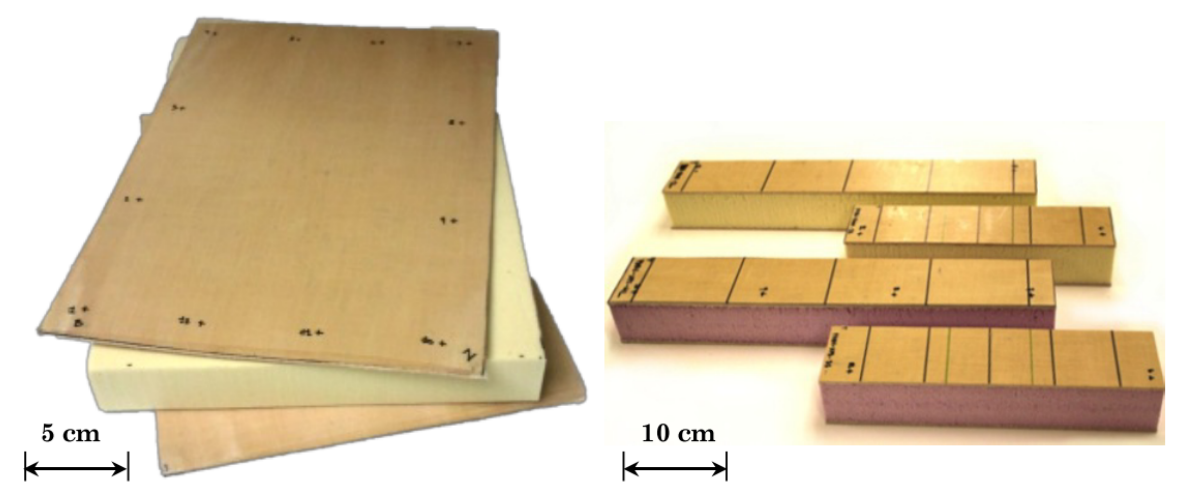

Figure 2.7: Fully and partially biobased sandwich panel specimens [38].

followed by 1440 minutes of exposure to a 90 humidity environment. It was found that the moisture exposed panels lost mass more rapidly and suffered a decrease in mechanical properties larger than the dry biocomposites. It is important to note that UV exposure and these environmental factors also cause synthetic composites to degrade, but typically at a rate and degree less severe than for bio based structures. It was concluded that untreated biocomposites are not a suitable replacement for building materials in most applications, although some potential roles (an example of short term emergency shelters was given) where short term usage and clean disposal are desired. It would seem that treatment to better control moisture absorption and degradation is a necessity for larger scale adoption of biocomposites, and more sustainable and ecologically friendly treatments are highly desirable for achieving this goal without compromising the ecological advantages of these materials.

PHB grafting: To reduce the brittleness and improve stress transfer and thermal stability of PHB-cellulose composites, a study by the Unversity of Idaho Renewable Materials Program used dicumyl peroxide (DCP) to graft the PHB to the cellulose [39]. PHB and commercial cellulose fiber (CF1) were coated separately with DCP in acetone, then dried, mixed, and injection molded into bars with $20 \%$ cellulose 
weight fraction. Total DCP concentrations were varied from $2 \%$ to $5 \%$ and reactive extrusion times between 5 and 15 minutes. It was found that the maximum graft yield occurred with 2\% DCP and 5 minutes, as shown in Figure 2.8.

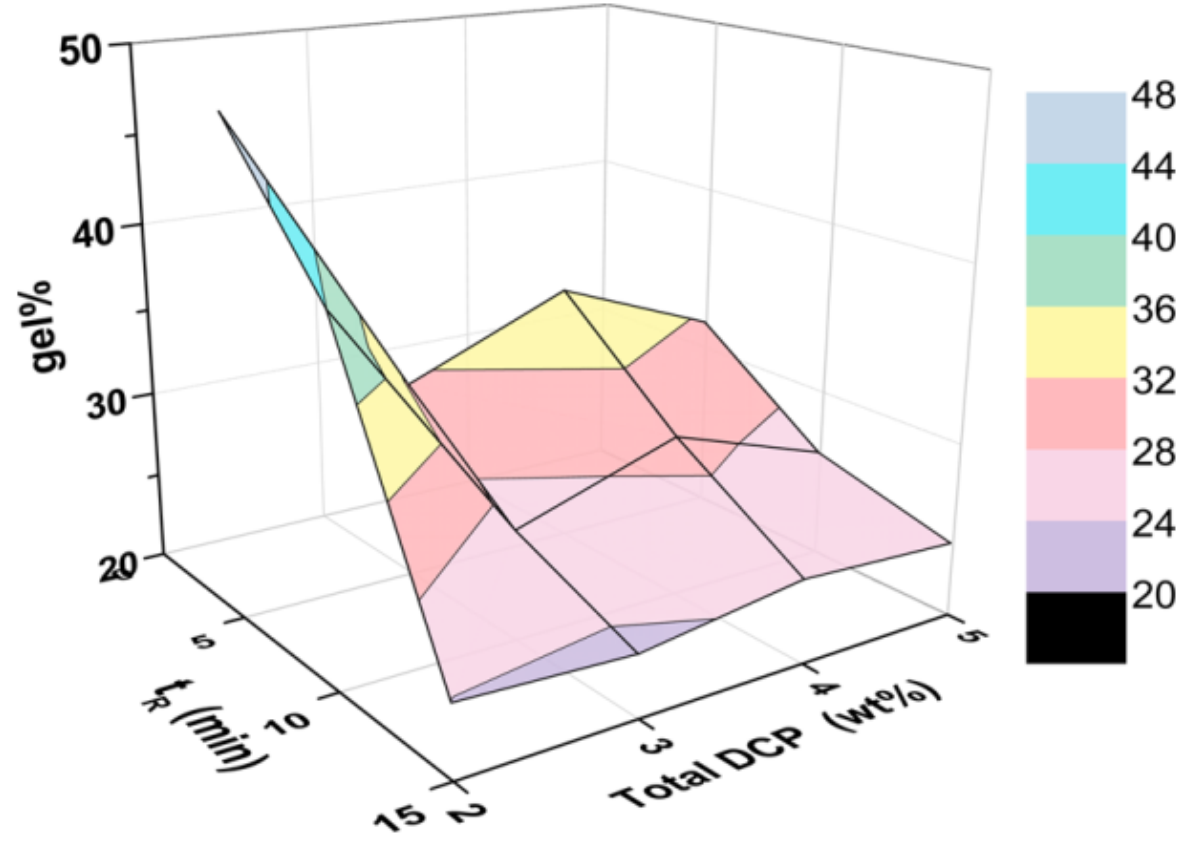

Figure 2.8: Effect of reaction time $\mathrm{t}_{R}(\mathrm{~min})$ and total DCP concentration on the graft yield (gel\%) of CF1 and PHB (CF1, $0.5 \mathrm{~g}$; PHB, $2 \mathrm{~g}$; temperature $=175{ }^{\circ} \mathrm{C}$ ) [39].

The graft yield was verified through electron paramagnetic resonance spectroscopy, and the impact on thermal stability was examined through differential scanning calorimetry, where it was found to have the highest glass transition temperature and crystallization temperature, indicating altered thermal stability. This may improve ease of processing of PHB and PHBV and shows potential for this treatment in biocomposites with other fibers. 


\section{EVALUATION OF LOCALLY SOURCED AGRICULTURAL RESIDUE IN COMPOSITES ${ }^{1}$}

\section{Contribution of Authors and Co-Authors}

Manuscript in Chapter 3: Evaluation of Locally Sourced Agricultural Residue in Composites

Author: Matthew Solle

Contributions: Manufactured samples, including silane and grafting treatment regimens, tested samples in flexure, created MATLAB code for analysis, examined samples with analysis of variance (ANOVA), fiber fraction analysis, and differential scanning calorimetry (DSC).

Co-author: Jesse Arroyo

Contributions: Acquired field emission scanning electron microscopy (FESEM) images of fractured composites, assisted in analysis of SEM results, assisted with DSC experimentation and results analysis, contributed to optimization of injection molding parameters.

Co-author: Stephan Warnat

Contributions: Developed initial design of experiments and ANOVA methods, provided guidance with and refinement of statistical analysis techniques.

Co-author: Macdonald Burgess

Contributions: Served as a contact with MSU BART Farm and local farmers. Acquired the agricultural residue, provided fiber processing equipment and facilities, and suggested storage, grinding, and other processing parameters. Provided guidance with statistical analysis.

Co-author: Cecily Ryan

Contributions: Primary advisor for the work. Contributed to analysis of all experimental results including flexural testing, fiber fraction analysis, SEM, DSC, and ANOVA.

\footnotetext{
${ }^{1}$ This chapter is a manuscript in preparation for submission to Composites Science and Technology.
} 


\section{Manuscript Information}

Matthew Solle, Jesse Arroyo, Stephan Warnat, Macdonald Burgess, Cecily Ryan Composites Science and Technology

Status of Manuscript:

$\boldsymbol{\otimes}$ Prepared for submission to a peer-reviewed journal

$\square$ Officially submitted to a peer-reviewed journal

$\square$ Accepted by a peer-reviewed journal

$\square$ Published in a peer-reviewed journal

Publisher: Elsevier, to be submitted 


\section{Introduction}

Biobased and biodegradable composites, or biocomposites, are a growing industry with potential application in green building, automotive, sporting equipment and goods, additive manufacturing, and other sustainable consumer products [3, 40,41]. The combination of biodegradable resins with natural fiber (NF) can offer a number of advantages over composites produced with synthetic fibers, including lighter weight, extremely low cost, and less wear on processing equipment [3]. The demand for biocomposites that incorporate NF is predicted grow, making it important to consider the long-term sustainability of NF fiber use in composites [27].

Research and usage of natural fibers typically falls into two categories. The first is NF grown for fiber production, selected for their high-performance mechanical properties. Bast fibers, including hemp, kenaf, jute, and flax, are most commonly used for these applications [5]. The second is residue or waste fibers from a variety of agricultural and forest processes, selected primarily for sustainability as fillers and substitutes in less mechanically demanding roles [13]. These fibers include leaf, seed, core and grass.

In addition to the mechanical properties of the fiber and resin themselves, one of the main factors that governs composite performance is the fiber-matrix interface. When combined, hydrophobic polymers and hydrophilic fibers suffer from poor interfacial adhesion, leading to decreased mechanical performance and load transfer across the interface [18]. Chemical treatments to the fiber or matrix can mitigate these issues, improving the performance and durability of biocomposites, including those where the fiber is selected primarily with sustainable production in mind $[19,20]$.

Here we investigate incorporating low-value agricultural residue from food crops into high-performing value-added bioproducts, such as these biobased composites. 
Agricultural residue from the following food crops was selected as the source for natural fiber reinforcement: (i) wheat with a hollow stem (Triticum aestivum 'Yellowstone'), (ii) wheat with a solid stem (Triticum aestivum 'Genou') bred in Montana and North Dakota for greater resilience against wheat stem sawfly infestation [42], (iii) barley (Hordeum vulgare 'Hocket'), and (iv) hemp (Cannabis sativa 'Finola') cultivated for seed and oil. As an industrially valuable and commonly used fiber, hemp's mechanical performance in composites served as a control for the food crop residues. This agricultural residue fiber (AF) was collected, ground and fabricated into biocomposites with a biodegradable resin, poly(hydroxybutyrateco-hydroxyvalerate) (PHBV) [17]. PHBV has mechanical properties similar to the commonly used non-degradable composite resin polypropylene [43]. It has been studied as a composite matrix for hemp and wheat fibers [12, 30, 38, 44, 45]. We investigate the impact of fiber fraction by fabricating composites with fiber weight percentages of $0 w t . \%, 10 w t . \%, 20 w t . \%$, and $30 w t . \%$.

To enhance mechanical performance and elucidate the importance of the fibermatrix interface in the biocomposites, we evaluated two fiber compatibilization treatments: (i) a silane coupling agent, (3-glycidoxypropyl)trimethoxysilane (GPTMS), selected for interfacial compatibility with PHBV and room temperature (reduced energy) processing $[21,22]$ and (ii) AF grafted with PHBV (AF-g-PHBV), selected to modify the fiber surface with PHBV, thereby creating a chemical bond at the interface. Both of these treatments were selected to retain the biodegradability of the composite as a whole, as PHBV-based NF composites have been previously shown to be biodegradable with fiber treatments similar to the ones which are investigated herein $[10,37]$. To assess the combined impact of different fiber types and treatments, a design of experiments was created to evaluate the significance of fiber fraction and treatments on the mechanical performance of the composites in flexure. In 
this analysis, AF from food production were compared with hemp, an established industrial fiber, but grown and collected as agricultural residue similar to the food crops to more accurately compare differences between materials. The untreated fibers at increasing weight percents establish a baseline for mechanical performance, serving as the comparison for potential enhancements with the two treatments (GPTMS and AF- $g$-PHBV). Fiber fraction analysis and differential scanning calorimetry (DSC) were used in conjunction with the mechanical test results to determine the effect of treatments. Composites were fabricated via bulk compounding and injection molding, to allow for rapid sample assembly in a consistent and controlled process. Manufacturing parameters in the compounder and injection molder were optimized pre-study on the selected materials to ensure even dispersion of all fiber types while minimizing partial mold fills. In this work, it was found that reducing the temperature and pressure to raise the viscosity of the melt can inhibit the movement of fiber. However, this can also lead to partial fills of the mold cavity, as a slow moving flow can cool prematurely. The purpose of the optimization was to find the lowest temperature and pressure for which the mold would fill reliably.

\section{Materials and Methods}

\section{Materials}

PHBV powder and pellets from TianAn Biologic Materials Co., People's Republic of China (product codes ENMAT Y1000 and Y1000P), were used for the composite matrix. Four agricultural residues were incorporated as natural short-fiber reinforcement into the biocomposites: (i) hollow stem wheat (Triticum aestivum 'Yellowstone'), (ii) solid stem wheat (Triticum aestivum 'Genou'), (iii) barley (Hordeum vulgare 'Hocket'), and (iv) hemp (Cannabis sativa 'Finola'), a common NF reinforcement used as a comparison fiber. Both wheat and barley 
were planted and harvested during the 2017 growing season at the Montana State University (MSU) Bozeman Agricultural Research and Teaching (BART) Farm. Hemp was grown in a nearby $(\sim 1.8 \mathrm{~km})$ privately owned and operated farm. All residue was collected by hand post-harvest; the hemp was collected approximately 2 weeks after the other fibers due to a later harvest. The residue fibers were kept in a drying oven at $50^{\circ} \mathrm{C}$ for 2 weeks, then ground in a Wiley Laboratory Mill Model 4 . Fibers passing through a $2 \mathrm{~mm}$ screen were collected for composites. Resin and fibers were kept in a desiccator prior to composite fabrication.

\section{Fiber-Matrix Compatibilization}

Un-modified fibers were compared to fibers with two types of coupling agents: (i) GPTMS and (ii) AF- $g$-PHBV.

Silane vapor deposition: (3-glycidoxypropyl)trimethoxysilane (GPTMS) (product code SIG5840.0) was acquired from Gelest, Inc., USA, and used without further purification. Vapor deposition of GPTMS on the fibers was based on a selfassembled monolayer method from Karakoy et al. and is a room-temperature method similar to the elevated temperature deposition demonstrated for PHBV-wood fiber composites. $[20,46]$. Fiber was spread in a thin layer on a foil-lined plate in a vacuum desiccator. A $0.1 \mathrm{~mL}$ drop of GPTMS was deposited into a weigh boat with a $1 \mathrm{~mL}$ syringe. The chamber was pumped under vacuum for 3 minutes, then the vacuum valve was closed, maintaining a vacuum of $740 \mathrm{mmHg}$ for 1 hour, after which the chamber was repumped and left for 1 more hour before the coated fiber was removed. The fiber was used for samples shortly after removal.

PHBV grafting to fiber: Dicumyl Peroxide (DCP), 99\% from Acros Organics (product code AC349881000) was used as a thermal free radical initiator to graft PHBV to the AF. Using a modified method of the grafting procedure developed by 
Wei et al. for cellulose grafted polyhydroxybutryate [39], DCP was added to $50 \mathrm{~mL}$ of acetone (HPLC Grade, Fisher Chemical, A949-4) and stirred until dissolved. AF and powdered PHBV were added and stirred in separate containers for 30 minutes. The weight ratio was 1:100 DCP:AF and 1:100 DCP:PHBV. After drying and mixing coating fiber and PHBV, the grafting process was initiated using reactive extrusion under a dry nitrogen purge for 5 minutes before injection molding the sample, following the fabrication procedure given below. These samples are denoted AFg-PHBV. Additional details are given in Appendix A.

\section{Fabrication of PHBV-AF Composites}

Dried fiber and resin were fed into a Thermo Scientific HAAKE Minilab II compounder, with resin first to prevent fiber agglomeration and jamming, and fiber added in gradually to improve dispersion and mixing. The compounder was run at a screw speed of $100 \mathrm{rpm}$ and a temperature of $150^{\circ} \mathrm{C}$ for 5 minutes to allow even mixing, verified by the stabilization of screw torque and internal pressure readings. In addition, a dry nitrogen purge of the mixing and sample loading chamber was run during the 5 minutes for AF- $g$-PHBV grafted samples.

The melt was then extruded into the injection gun of a Thermo Scientific HAAKE Minijet Pro injection molder, where it was immediately injected into an 60x10x $1 \mathrm{~mm}$ bar mold. The mold temp was set to $40^{\circ} \mathrm{C}$ to cool the sample while ensuring a complete fill. The initial pressure for $0 w t . \%$ to $20 w t . \%$ samples was 250 bar. The $30 w t . \%$ samples required a higher initial pressure of 450 bar to ensure a complete fill of the mold. All samples were held at a post injection pressure of 150 bar for 5 seconds before the sample was removed from the mold. Samples were kept in a desiccator for 7 days prior to flexural testing to minimize material differences due to aging, a significant factor to account for when using PHBV [47]. 
Flexural Mechanical Testing

Samples were mechanically tested in flexure per ASTM D7264 Procedure B [48]. An Instron 5543 single column tabletop tensile tester with a $1 \mathrm{kN}$ load cell was used with an Instron 4 point bending fixture (Part No. 2810-405). All samples were tested at a strain rate of $1 \mathrm{~mm} /$ minute. The support span was set to $40 \mathrm{~mm}$, meeting the ASTM specified span-thickness ratio of 40:1 for the samples used. All failures occurred in a manner consistent with the failure modes given in D7264 11.7. Flexure testing was selected as the preferred method of determining mechanical properties as it provides a more realistic approximation of real world mechanical loading and a more complex characterization of the material. Tensile testing utilizes a unidirectional stress state and creates a concentration at the center of the testing sample, while 4 point flexure testing creates a uniform stress state across the composite between the points.

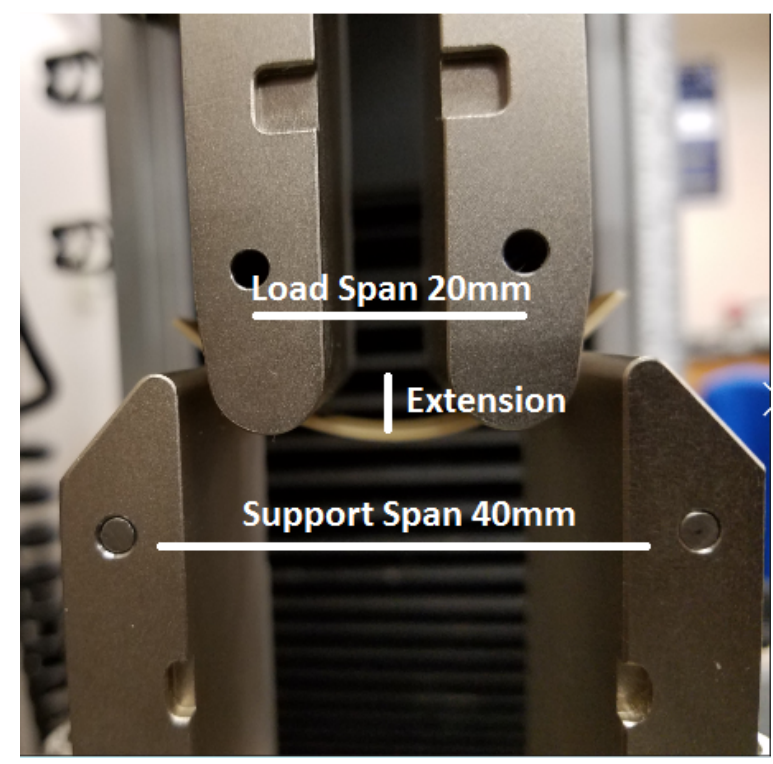

Figure 3.1: Flexural test setup with sample and annotations.

Mechanical properties were determined through Matlab analysis of the Instron 
testing data. The maximum load, extension at maximum load, and extension at break were taken directly from the test data, then used to calculate the ultimate flexural strength (UFS), the flexural secant modulus, the flexural chord modulus, strain at UFS, and elongation at break. These properties are calculated using the following formulas [48]. For stress and strain:

$$
\begin{aligned}
\sigma & =\frac{3 P L}{4 b h^{2}} \\
\epsilon & =\frac{4.36 \delta h}{L^{3}}
\end{aligned}
$$

For chord and secant modulus:

$$
\begin{aligned}
E_{f}^{\text {chord }} & =\frac{\Delta \sigma}{\Delta \epsilon} \\
E_{f}^{\text {secant }} & =\frac{0.17 L^{3} m}{b h^{3}}
\end{aligned}
$$

$\sigma$ is the maximum stress at a point, $\epsilon$ is the maximum strain at the point, $P$ is the applied force in Newtons, $L$ is the support span in $\mathrm{mm}, b$ is the width of sample in $\mathrm{mm}$, and $h$ is the thickness of sample in $\mathrm{mm} . E_{f}^{\text {chord }}$ is the chord modulus, found as the gradient of stress and strain across a predetermined stress range. ASTM D7264b recommends the gradient of $\epsilon=.001$ to $\epsilon=.003$. which was used for this study. $E_{f}^{\text {secant }}$ is the secant modulus, which determines modulus from $m$, the slope of the secant of the force-deflection curve at any chosen range. Matlab was used to determine $m$ by finding the first consistently linear portion of the force-deflection curve and using that to calculate the secant modulus (See Appendix B). 
$\underline{\text { Rule of Mixtures Model }}$

To compare this data to calculated values using "Rule of Mixtures" (ROM) model, equation 2.4 was used to calculate Young's modulus for barley, wheat, and hemp fiber composites at weight fractions of $10 \%, 20 \%$, and $30 \%$, using material parameters from previous work $[5,23-26,49]$.

\section{Statistical Design of Experiments}

To clearly separate small variations in the sample data from larger trends, the experiment was designed as a three degree of freedom analysis of variance (ANOVA), with F test significance defined as 99\% confidence [50]. Samples of the same treatment, fiber, and fraction were produced in three separate runs spread out to reduce the influence of uncontrolled variables in the production process. The difference in strength and modulus between different fibers at the same treatment and weight fraction was examined, as was the difference in strength and modulus for the same fiber with different treatments and weight fractions.

\section{Material Characterization}

Fiber fraction analysis: To examine the variation in fiber weight percent due to processing (compounding and injection molding), fiber fraction analysis was done as per ASTM 3171, with chloroform acting as the hot liquid medium [51]. Flexure tested samples were placed in a beaker with $50 \mathrm{ml}$ chloroform, then stirred at $65^{\circ} \mathrm{C}$ for one hour underneath a condensation tower. The dissolved mixture was then poured through a cellulose filter under vacuum to separate the chloroform/PHBV solution from the fiber. Fiber was collected, dried at $50^{\circ} \mathrm{C}$, and weighed. Fiber 
weight percentage was calculated as follows:

$$
w t . \%=\frac{\text { Filter Weight }(\text { Post FFA })-\text { Filter Weight }(\text { Empty })}{\text { Sample Weight }} * 100 \%
$$

Differential Scanning Calorimetry: To examine the extent to which DCP modified the PHBV matrix and fibers, DSC was run on a small set of samples. A TA Instruments DSC 2920 (Serial Number 125827) was used to assess the impact of the PHBV grafting treatment. Samples included PHBV powder, PHBV powder modified with DCP (no reactive extrusion), reactively compounded and molded PHBV samples with DCP ( $g$ PHBV), grafted composite with $30 \%$ hollow stem wheat (AF- $g$-PHBV), and untreated composite with $30 \%$ hollow stem wheat.

DSC, with a nitrogen flow rate of $50-54 \mathrm{~mL} / \mathrm{min}$ as optimized in previous work [37], was used to analyze the bioplastic and composites. Samples were encapsulated in aluminum pans with a target sample weight of $5 \mathrm{mg} \pm 2 \mathrm{mg}$, and heated from $25^{\circ} \mathrm{C}$ to $180^{\circ} \mathrm{C}$ in the first heating cycle at a rate of $10^{\circ} \mathrm{C} / \mathrm{min}$. After equilibrating to $180^{\circ} \mathrm{C}$, they were held at $180^{\circ} \mathrm{C}$ for 2 minutes prior to cooling at $10^{\circ} \mathrm{C} / \mathrm{min}$ to $25^{\circ} \mathrm{C}$, which is below the crystallization temperature for PHBV and these composites. The samples were then heated at $10^{\circ} \mathrm{C} / \min$ to $200^{\circ} \mathrm{C}$ in the second and final heating cycle. This procedure was designed due to cooling limitations below room temperature, and successfully cooled past crystallization before the 2nd heating cycle. The glass transition temperature $\left(T_{g}\right)$ was taken to be the midpoint of the heat capacity change, the melting temperature $\left(T_{m}\right)$ was measured as the minimum of the endothermic peak, and the crystallization temperature $\left(T_{c}\right)$ was taken as the maximum temperature of the exothermic peak upon cooling (in between the first and second cycles).

The percent crystallinity of the PHBV matrix, $\chi$, was determined using the 
following equation [52]:

$$
\chi[\%]=\frac{\Delta H_{m}-\Delta H_{c}}{\Delta H_{m}^{\circ}\left(1-W_{f}\right)} \cdot 100 \%
$$

where $\Delta H_{m}$ and $\Delta H_{c}$ are the enthalpies of melting and crystallization measured upon heating, $W_{f}$ is the weight fraction of fiber of the composite, and $\Delta H_{m}^{\circ}$ is the reference value for $100 \%$ crystalline poly(hydroxybutyrate) (PHB), $146 \mathrm{~J} / \mathrm{g}$ or 12.5 $\mathrm{kJ} /$ mole $[53,54]$. As a reference, a typical value for the crystallinity of annealed PHB samples measured by Barham et al. was $86 \%$ [53].

Scanning Electron Microscopy: Examination of fracture surfaces though field emission scanning electron microscopy (FESEM) was done on a Supra 55VP System 2512 at $1 \mathrm{kV}$ with an SE2 detector. Samples were uncoated with images taken of the break surface. Fiber and matrix surfaces were characterized and the fiber-matrix interfaces were imaged along with assessing potential failure modes.

\section{Results and Discussion}

\section{Rule of Mixtures}

The calculated prediction for hemp was close to the actual experimental values for hollow stem wheat, barley, and hemp. However, predictions for barley and wheat were lower than predicted, suggesting that the modulus values for these fibers from the literature were lower than the actual performance of the material in a composite. A previously reported value of Young's modulus was used in the prediction [49], which was lower than experimental measurements of flexural modulus and lead to further shifting of the predicted modulus downward. 


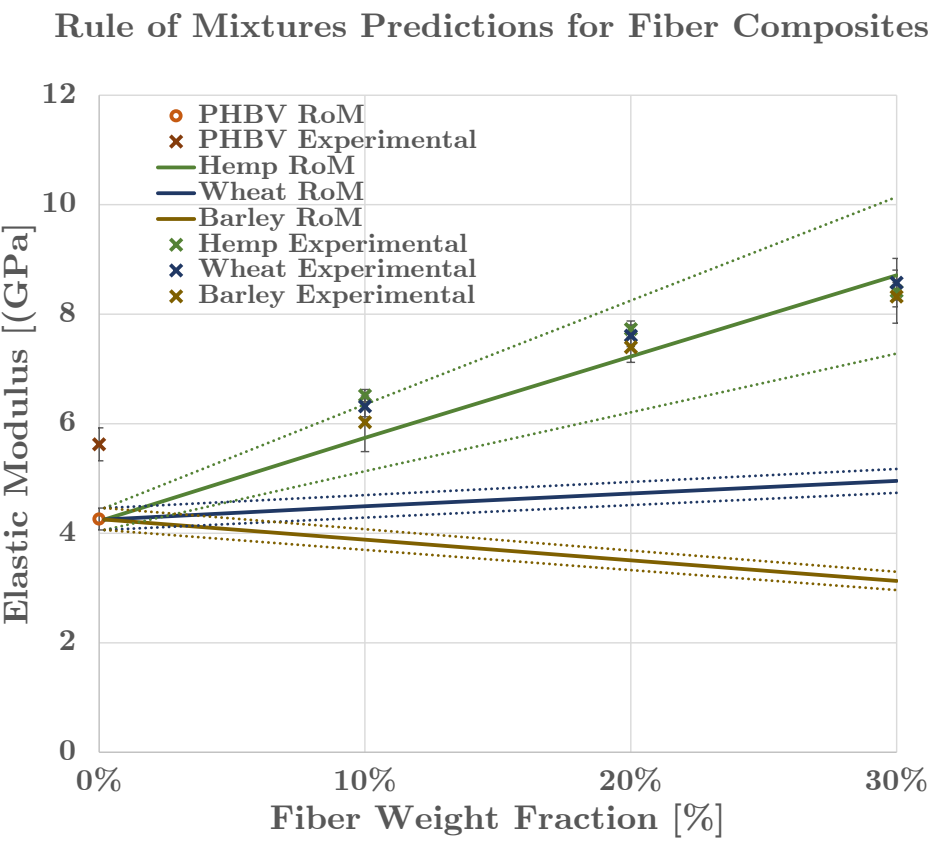

Figure 3.2: Rule of mixtures compared to experimental values for untreated samples. Lines are the prediction of modulus using Equation 2.4 (dotted lines indicate the variation in the fiber modulus values reported in literature), and X's are mean experimental values for untreated fiber samples.

\section{Mechanical Properties}

The responsiveness of mechanical performance to fiber fraction and treatments was dependent on the property (flexural modulus, UFS, strain at break) and to an extent, fiber type and treatment. Some of the fibers and treatments could be statistically differentiated while others exhibited comparable behavior. To analyze and summarize the results we compared (i) the effectiveness of the compatibilization treatments for each fiber type, (ii) the mechanical properties of different fibers 
undergoing the same treatment, and (iii) the overall effectiveness of the treatments for AF fibers by aggregating all fiber types.

Comparison of treatments for each fiber: Silane treated hemp composites had a higher modulus than untreated hemp or grafted samples, regardless of fiber fraction (Figure 3.3a.). For barley, all three treatments performed similarly at $10 \%$ and 20\% fiber, but GPTMS and AF-g-PHBV samples at 30\% had statistically significant increase in modulus over that of the untreated samples (Figure 3.3b.). The moduli of solid stem wheat composites were consistently highest after silane treatment and weakest after grafting (Figure 3.3c.). Hollow stem wheat samples had a statistically indistinguishable modulus between treatments at 10\%, but GPTMS samples had a higher modulus at all other fiber fractions (Figure 3.3d.). Overall, silane treatment was most effective in increasing the modulus in hemp and solid stem wheat composites.

In terms of ultimate flexural strength, silane treatment had a significant increase over untreated samples of hemp and hollow stem wheat (Figure 3.4a.). DCP samples were not consistent enough to establish trends, as some of the highest and lowest values of UFS occured in DCP treated samples. For example, hemp- $g$-PHBV at a $30 \%$ weight fraction had the mean for UFS of any test set, but with a confidence of 95\%, did not meet the pre-established threshold for significance when compared to silane treated hemp. Hemp showed the most clear trend in increasing UFS with fiber fraction and treatments of all of the fibers.

The high degree of variability may have been caused by changes in the grafting efficiency of the DCP reaction. This reaction is a time and temperature sensitive process which is dependent on the degree to which DCP reacts prior to fiber/polymer mixing and how long the mixture is given to react during compounding before injection molding. The percentage of DCP and reaction time were selected based 
on the results from Wei et al., but variations in the amount of hydroxyl groups on the fiber surfaces as compared to cellulose and processing parameters may have lead to a higher or lower grafting efficiency than obtained by the previous study [39].

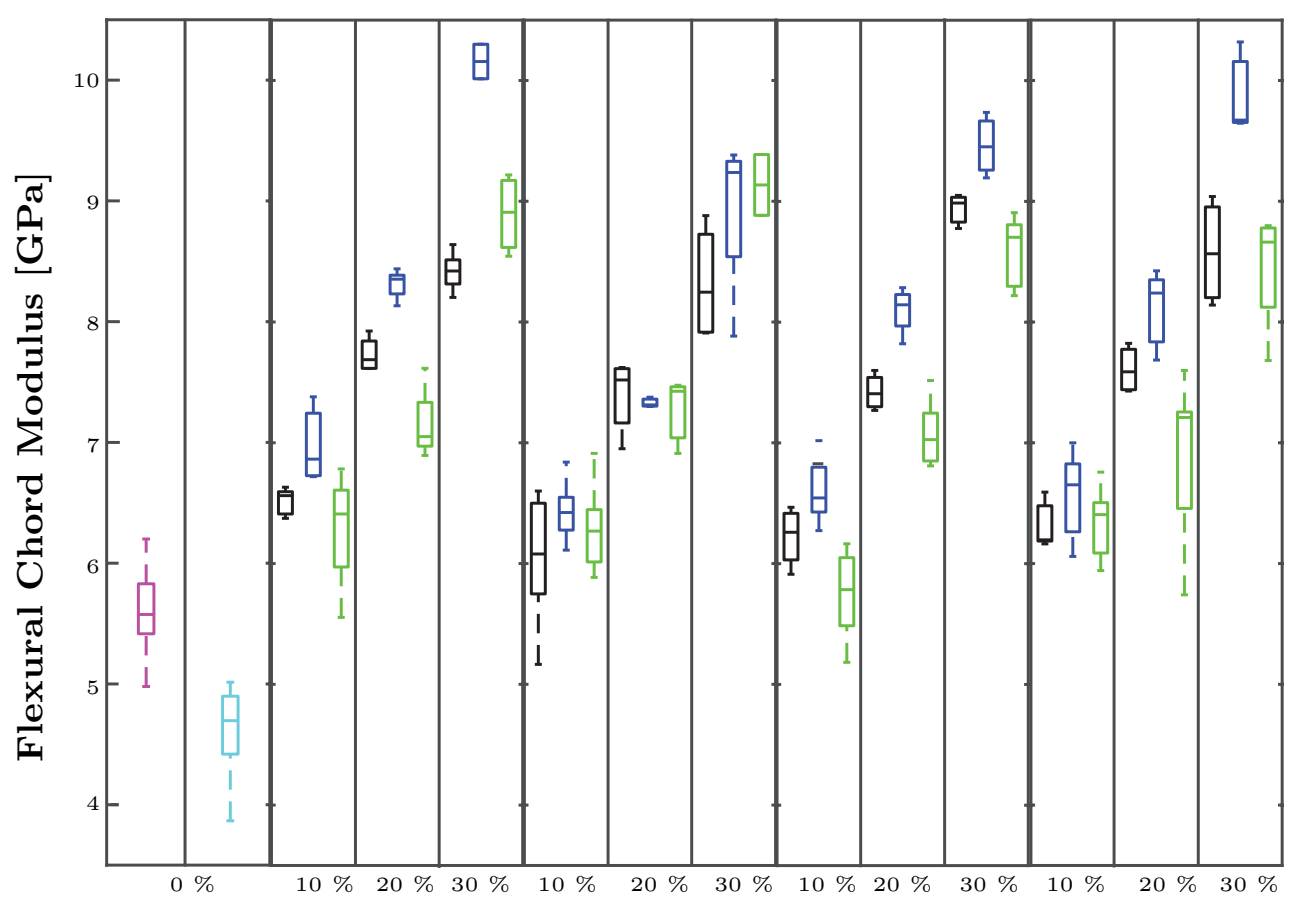

Fiber Fraction \%
(a) PHBV
(b) Hemp
(c) Barley
(d) Solid Stem (e) Hollow Stem

- PHBV

$-g \mathrm{PHBV}$

— Untreated - Silane

- AF- $g$-PHBV

Figure 3.3: Chord modulus of all fibers for each weight percentage, including treatments. Magenta is neat PHBV, teal is $g$ PHBV, black is untreated, blue is silane, green is grafted.

Comparison of different fibers by treatment: $99 \%$ confidence analysis showed that hemp samples were significantly more rigid than solid stem wheat in 8 of 9 combinations of fiber fraction and treatment, with the only exception being untreated 


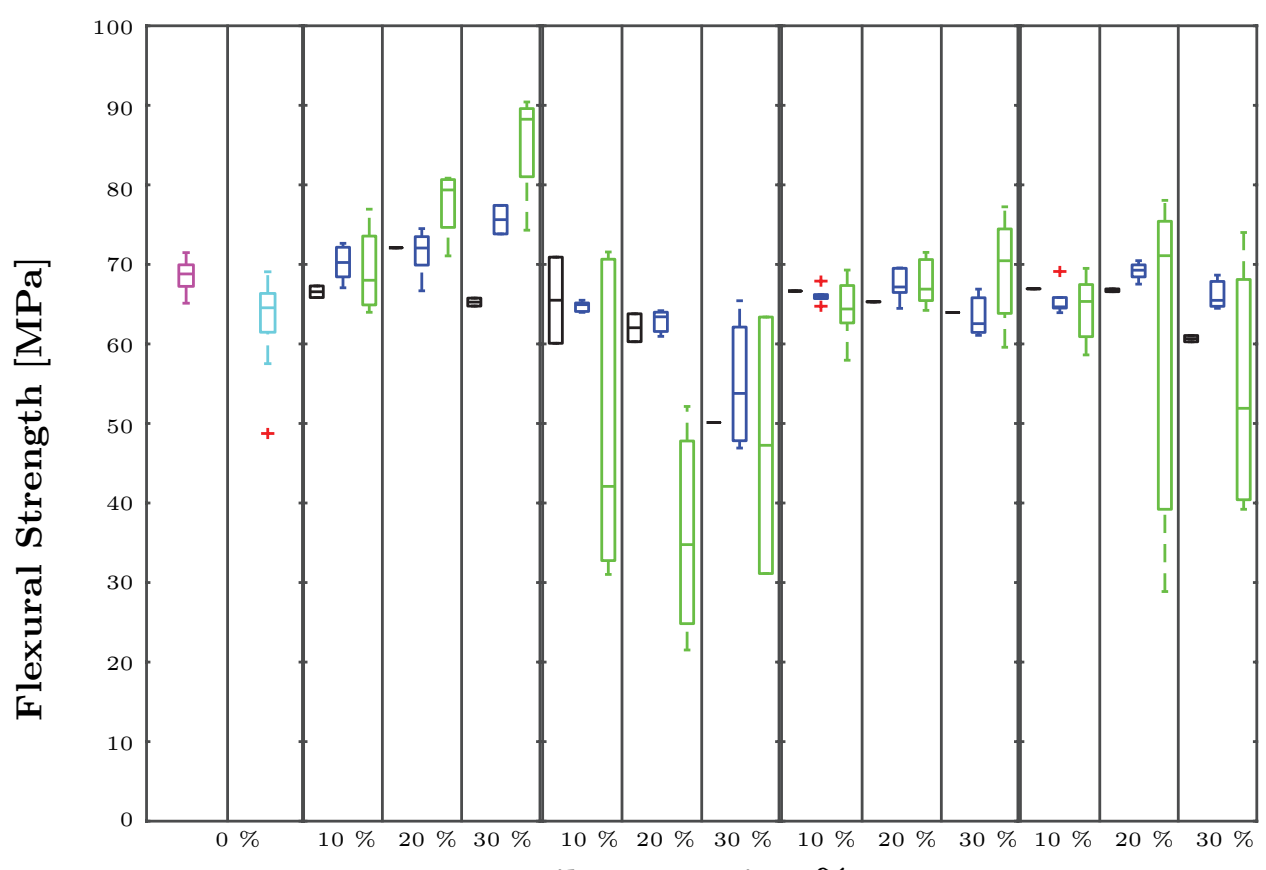

Fiber Fraction \%
(a) PHBV
(b) Hemp
(c) Barley
(d) Solid Stem
(e) Hollow Stem

- PHBV

$-g \mathrm{PHBV}$

—Untreated — Silane

- AF- $g$-PHBV

Figure 3.4: Ultimate flexural strength of all fibers by fiber, including treatments. Magenta is neat PHBV, teal is $g$ PHBV, black is untreated, blue is silane, green is grafted.

samples at 30\%. Hemp's rigidity in comparison to the other fiber composites was less consistent. Barley- $g$-PHBV composites were not distinguishable from hemp at $10 \%$ weight fraction, and higher than hemp at $20 \%$ and $30 \%$. Hollow stem wheat composites had the closest stiffness to hemp composites, with the majority of samples of both fiber types being statistically indistinguishable from each other. Interestingly, hollow stem composites were more rigid than solid stem composites in all cases where a statistically significant comparison could be made, although differences with $10 \%$ 
and $20 \%$ silanated samples were too close to distinguish.

The flexural strength of hemp composites was higher with DCP and silane treatment than other fibers with the same treatments and fiber fractions. The closet agricultural residue comparison was hollow stem wheat, which had similar modulus and strength to hemp at fiber fractions of $10 \%$ and $20 \%$. However, hemp was primarily set apart from the agricultural residues by being the only fiber which consistently increased in flexural strength as fiber fraction increasing to $30 \%$, with the highest flexural strength of all samples occurring at 30\% silane treated hemp, $20 \%$ grafted hemp, and 30\% grafted hemp (Figure 3.4).

Despite collecting the hemp for this study as an agricultural residue for seed and oil production, we anticipated that its fiber structure as a bast fiber would lead to improved fiber performance as compared to the other agricultural residues. Among the agricultural fibers used, hollow stem wheat had similar performance to hemp, with chord modulus that was not statistically distinguishable from hemp in 7 of 9 combinations of weight fraction and treatment, and similar ultimate flexural strength at low fiber weight fraction.

Aggregate fiber evaluation: To examine the overall effectiveness of the fiber treatments and the impact of increasing fiber fraction, all samples were combined into an aggregate set differentiated by fiber weight percentage and treatment (Figure 3.5). This analysis indicated that GPTMS treated samples had the highest chord and secant modulus (3.5a. and b.). AF- $g$-PHBV samples had lower modulus values and reached their maximum stress at a lower strain than other samples (Figure 3.5). 

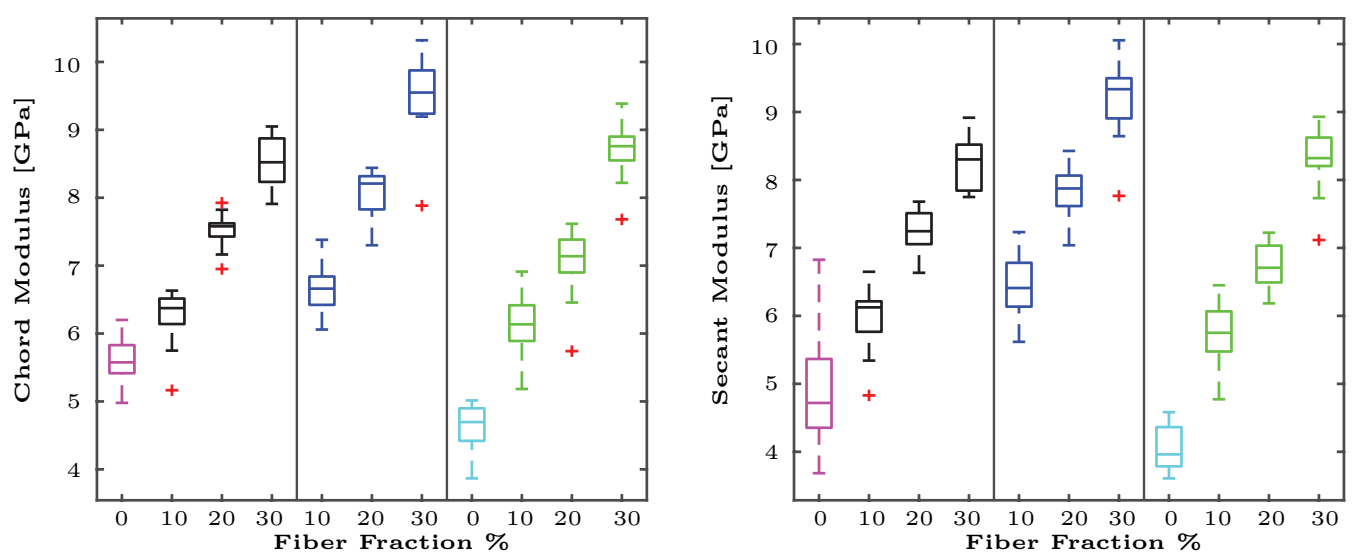

(a)

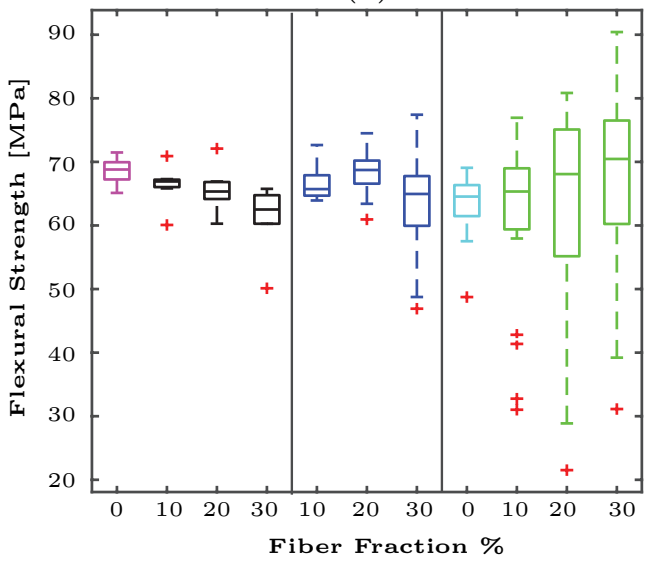

(c)

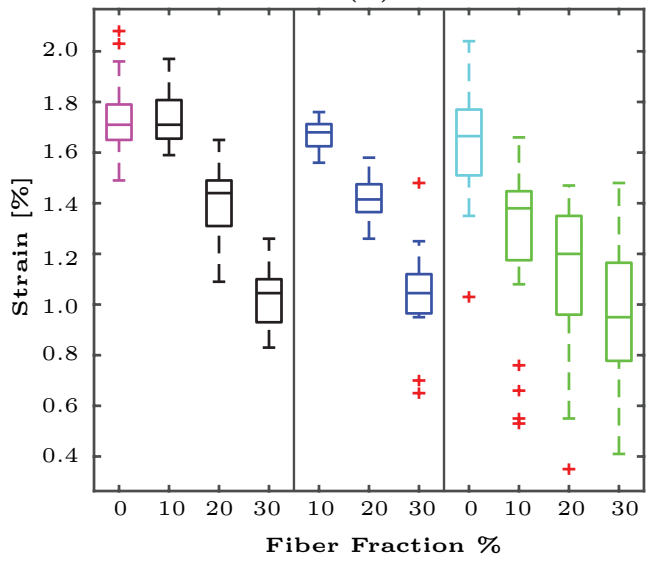

(d)

$$
\text { - PHBV }-g \text { PHBV } \quad \text { Untreated - Silane - AF- } g \text {-PHBV }
$$

Figure 3.5: Mechanical properties of the combined fiber set for each weight percentage, including all treatment regimens. Magenta is neat PHBV, teal is $g \mathrm{PHBV}$, black is untreated, blue is silane, green is grafted. 
However, the $g$ PHBV used to make the AF- $g$-PHBV samples has lower base UFS and modulus than the neat PHBV used for the untreated and AF- $g$-PHBV samples (Figure 3.5a-c., magenta). To account for this difference in the matrix, Figure 3.6 shows the increase in modulus with increasing fiber fraction normalized by the matrix modulus as well as the percent change for these increases. After normalizing for matrix values, the addition of $\mathrm{AF}-\mathrm{g}$-PHBV results in larger increases in modulus as compared to untreated and AF- $g$-PHBV samples.

With the sample set examined as a whole, there was not a significant difference in flexural strength with the addition of fiber or with treatment (Figure 3.3c.). Observable trends did exist in hemp and hollow stem wheat samples, but inconsistency in barley and solid stem wheat obscured these trends. Strain at the point of maximum flexural strength decreased with increasing fiber fraction, but was not significantly affected by treatment (Figure 3.3d).

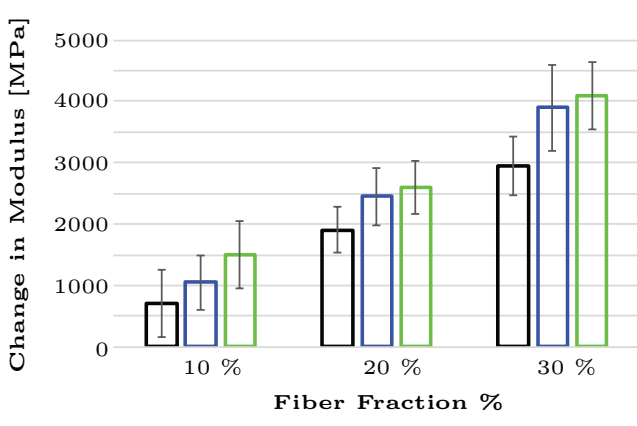

(a) Change in Modulus

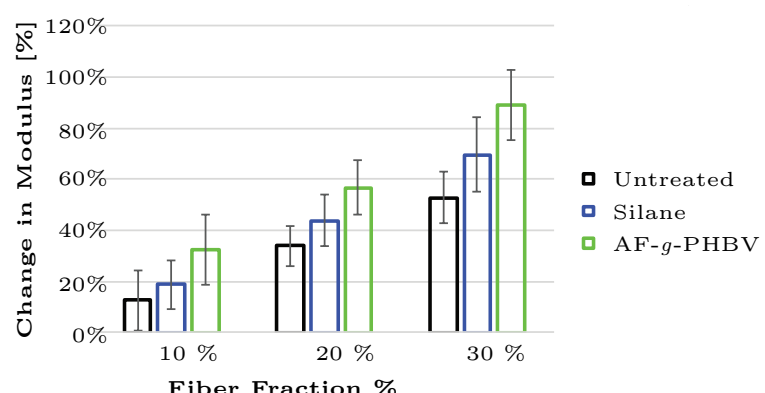

(b) Percent Change in Modulus

Figure 3.6: The change in chord modulus caused by adding fiber with different treatments. Black is untreated, blue is silane, and green is grafted. 
Materials Characterization

Fiber Fraction Analysis: The average difference between measured fiber fraction and expected fiber fraction was $4.3 \%$, equivalent to a 0.03 gram difference in an average 0.77 gram sample. Higher fiber fractions had lower average differences between measured and expected fiber fractions, likely due to an increased sensitivity of lower fiber fractions to small amounts of PHBV residue remaining on the filers and fibers. Overall, measured fiber fraction values did not correlate with measurable differences in mechanical properties at each fiber fraction. The full results can be found in Figure A.10.

Differential Scanning Calorimetry: Figure 3.7 shows the melting temperature of powdered PHBV, DCP treated PHBV prior to thermal processing, and injection molded $g$ PHBV. The melting temperature, $T_{m}$, indicates crystallite size and perfection. The first melting cycle for the PHBV and DCP treated PHBV have a melting peak at the same location, but after a temperature cycle, the DCP treated PHBV has a split melting peak shifted to a lower temperature indicating decreased crystallite size and order, likely due to crosslinking and molecular weight reduction in the matrix. This shift is similar to what has been observed in other related studies $[37,39,55,56]$.

The injection molded sample, $g$ PHBV, shows a $T_{m}$ shift lower than that of neat PHBV, but in between the peaks of the DCP treated PHBV, potentially indicating that the extent of the crosslinking reaction is less in the injection molded sample. A similar shift has been observed in another study using the same treatment process [39]. This decrease may indicate that a higher processing temperature would be beneficial in crosslinking PHBV with AF. Similarly the crystallization temperature, Figure 3.8 is impacted by crosslinks in the matrix.

Figures 3.8 and 3.9 show the crystallization and melting peaks in the second 


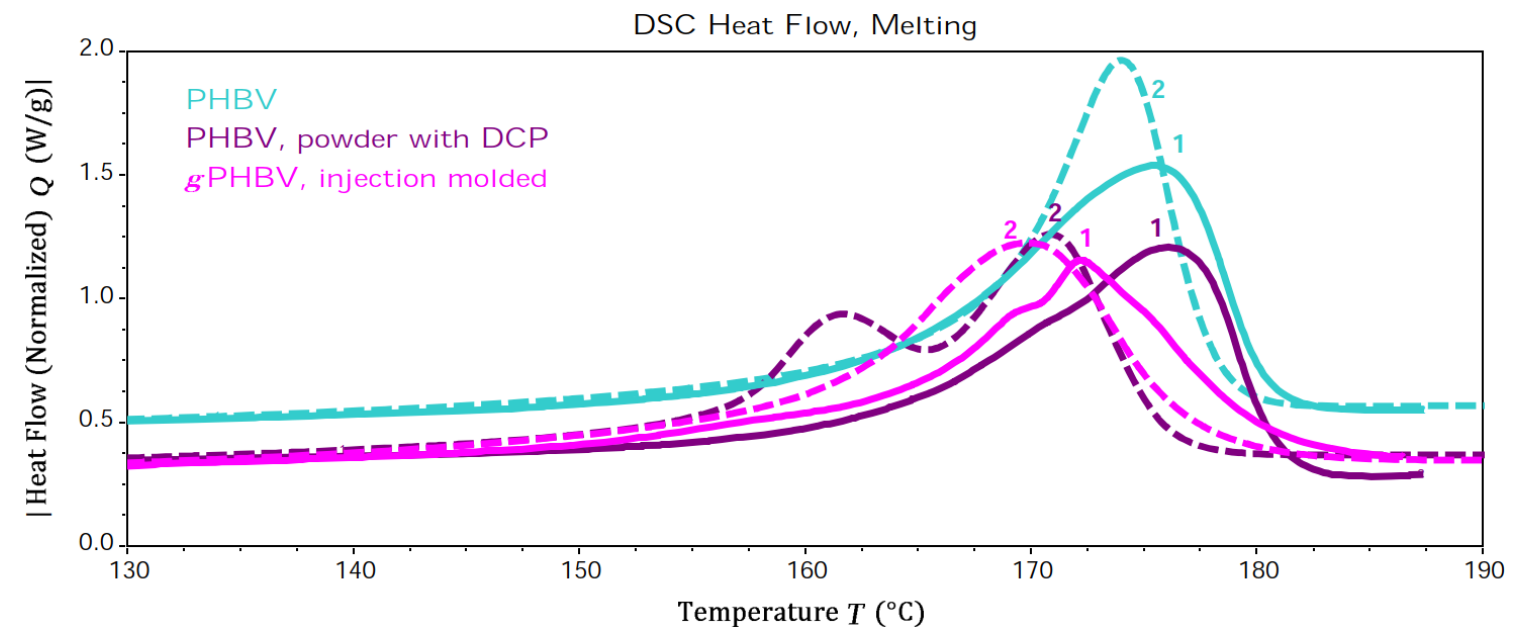

Figure 3.7: Differential scanning calorimetry showing the melting peak of powdered PHBV, DCP treated PHBV prior to thermal processing, and injection molded $g \mathrm{PHBV}$.

cycle of $20 \%$ HS wheat composites including all treatment regimens. The lower second melting peak in the AF- $g$-PHBV composite is likely related to interactions with the fiber component, including crosslinking reactions [20]. The "weak grafted" sample displays a significantly larger shift in crystallization and melting temperatures, and a more pronounce split peak. These shifts, combined with the large change in tested properties indicates a change to the matrix may be responsible for the reduction in strength. Full DSC results can be found in Appendix A, Table A.2. 


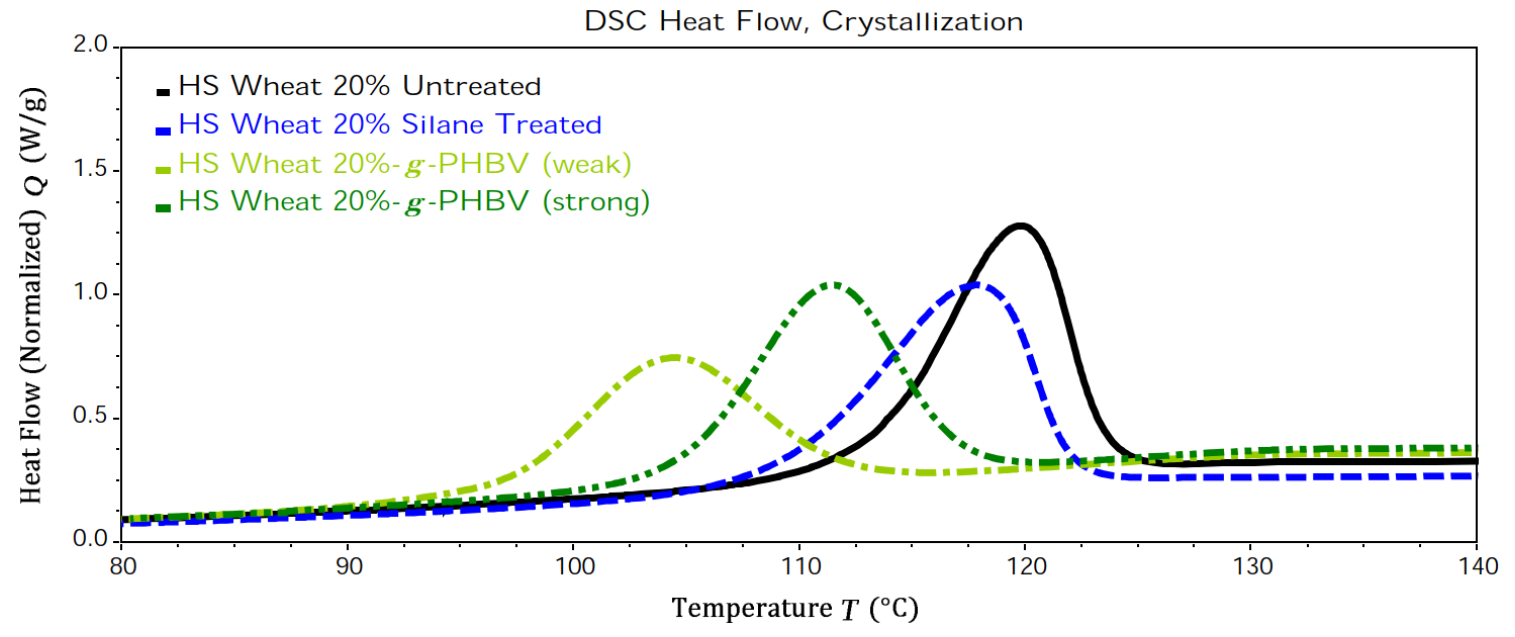

Figure 3.8: Differential scanning calorimetry showing the crystallization peak of $20 \%$ hollow stem wheat untreated, silane treated, weakly grafted, and strongly grafted samples.

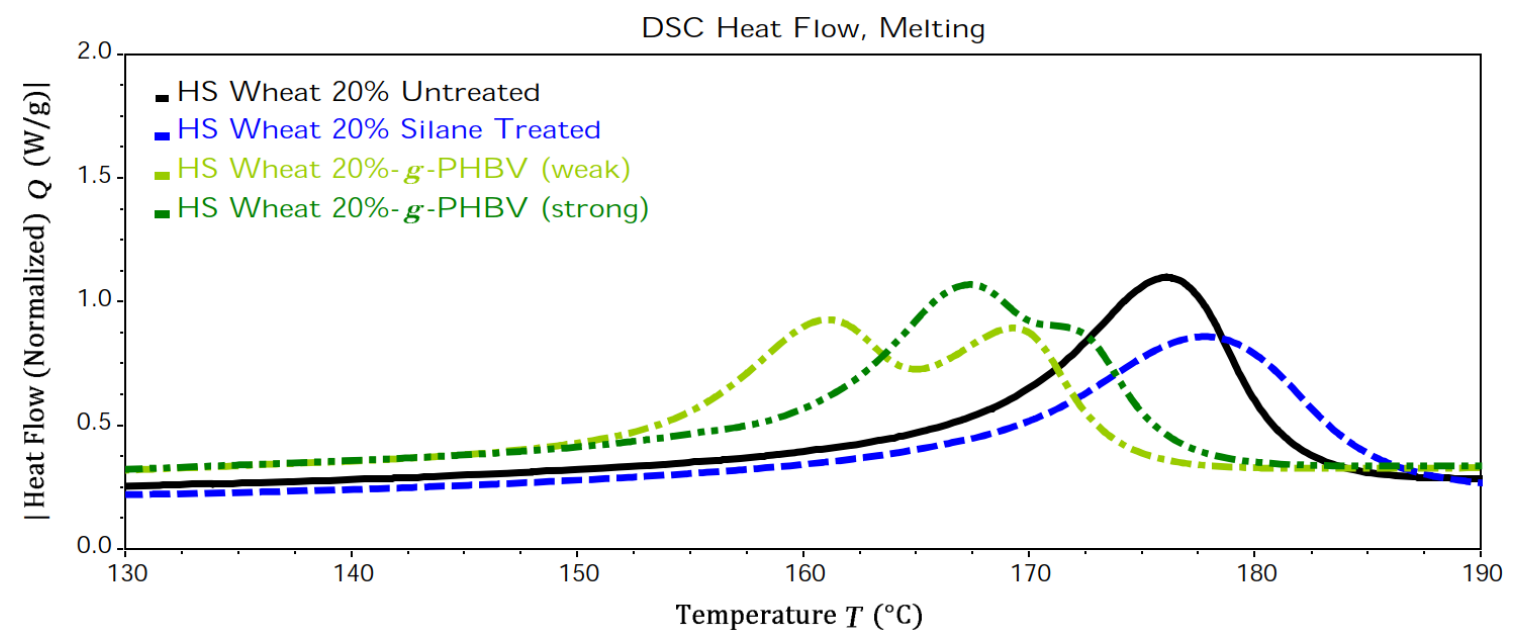

Figure 3.9: Differential scanning calorimetry showing the melting peak of $20 \%$ hollow stem wheat untreated, silane treated, weakly grafted, and strongly grafted samples. 
Scanning Electron Microscopy: A visible change in the PHBV matrix could be seen in the grafted composites $(3.10 \mathrm{c}, \mathrm{g})$. The matrix appeared to have sheared off in large, flat pieces in the low strength grafted sample. This altered matrix structure may have contributed to reduced mechanical properties in some of the grafted samples. The "strong grafted" sample displays areas with the smoothfractured matrix structure, but the difference is less apparent than with the weak sample. The degree of matrix alteration may be directly related to the change in mechanical properties. Fiber pullout seems to be a common failure mode of the composites, although some damage to exposed fibers indicating fiber breakage could be seen.

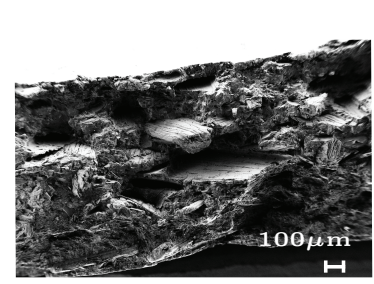

(a) Untreated

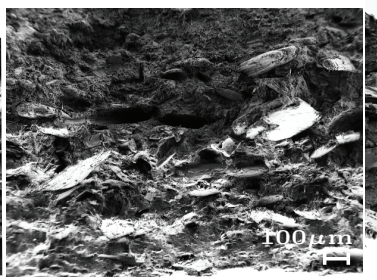

(b) Silane Treated

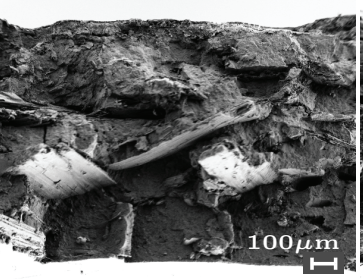

(c) AF- $g$-PHBV (W)

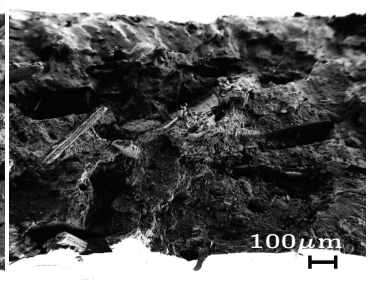

(d) AF- $g$-PHBV (S)

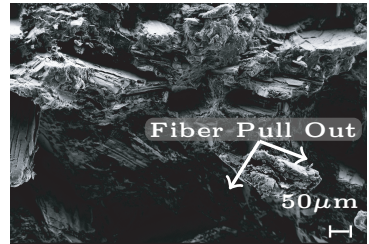

(e) Untreated

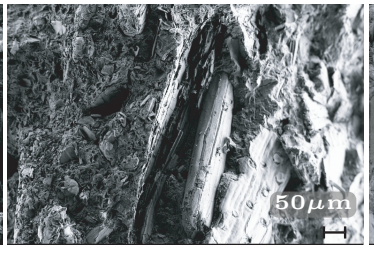

(f) Silane Treated

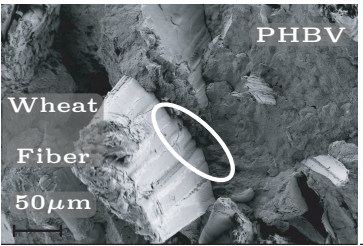

(g) $\mathrm{AF}-g$-PHBV $(\mathrm{W})$

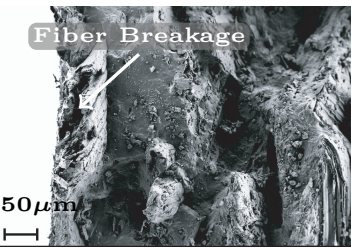

(h) AF-g-PHBV (S)

Figure 3.10: FESEM results for 20\% hollow stem wheat samples, where (a) is untreated, (b) is silanated, (c) is a "weak grafted" sample, and (d) is a "strong grafted" sample taken at low magnification. Sub-figures (e)-(h) are higher magnification images of the same composites. 


\section{Conclusions}

Flexural chord modulus and ultimate flexural strength were the primary measures for assessing the performance of composite samples, the impact of fiber type, treatment, and weight fraction. For all fibers and treatments, increasing fiber weight fraction from $0 \%$ to $30 \%$ increased the flexural modulus by a statistically significant amount with confidence of $99 \%$ or greater. Flexural modulus varied considerably based on the fiber type and treatment used. $30 \%$ hemp and hollow stem wheat composites treated with silane were the most rigid, with a significant difference over every other composite at $99.9 \%$ confidence. The difference between fiber types is small or not statistically significant for most untreated composites, while grafted samples with barley were more rigid than grafted samples with hemp. Silane treatment increased the modulus most consistently compared to untreated samples, and appears to be the most effective treatment if increasing modulus is a desired property.

In terms of ultimate flexural strength, fiber weight fraction had a less consistent relationship, with no measured significant difference in barley or solid stem wheat composites and a decrease in strength in untreated and hollow stem wheat- $g$-PHBV composites. For hemp, flexural strength increased in the treated hemp composites, but was not a factor for other fibers, suggesting that fiber type and treatment appear to be the most important factors for controlling flexural strength. Agricultural residue composites are similar in strength to hemp without treatment, but hemp with treatment was consistently stronger than any other fiber composite. The high

amount of variability in measured flexural strength in these samples and the lack of identifiable trends with increasing fiber fraction suggest flaw driven behavior in these samples which causes premature failure. 
The maximum flexural strength was achieved with $30 \%$ hemp composites treated with DCP, although 20\% hemp composites with DCP and 30\% hemp composites treated with silane also had high strengths. The most rigid sample combinations would be hemp or hollow stem wheat composites treated with silane at a weight fraction of $30 \%$. If the ability to resist a higher deformation is desired, then hemp at $10 \%$ weight fraction treated with silane has high rigidity and strength without a detectable reduction in max strain. The optimal combination for high strength and high stiffness is $30 \%$ hemp treated with silane. Hollow stem wheat performs most similarly to hemp composites in terms of chord modulus, but at a reduction in flexural strength relative to hemp, which may be significant depending on application.

Analysis of the FESEM and DSC results of grafted samples indicate a clear change to the matrix of the fiber, occurring in both grafted samples but to a greater extent in some samples, leading to a significant reduction in flexural strength. The cause for this change and the mechanisms by which the matrix is weakened will require additional research and examination to determine. As grafting efficiency has a known time and temperature dependence, small variation in processing between grafted samples may contribute to this decrease. [39]. 


\section{CONCLUSIONS AND DIRECTIONS FOR FUTURE WORK}

\section{Results of this study as related to the research objectives:}

1. Determine if agricultural residue fibers can be a mechanically competitive replacement for short, chopped industrial fibers (e.g. hemp) in biocomposites.

When treated with silane, hemp biocomposites had higher mechanical performance than agricultural residue fibers, particularly at high fiber fractions. Hemp treated with DCP had higher strength with a reduction in modulus. However, certain combinations of fiber fraction and treatment of agricultural residue composites did outperform hemp composites under the same conditions. For example, barley grafted samples had a higher modulus than hemp at $20 \%$ and $30 \%$ fiber, while $30 \%$ untreated solid stem wheat composites had a statistically higher modulus over any other untreated composite. Flexural properties were chosen for testing in this study, but other important properties for engineering biocomposites such as impact strength and thermal stability are important to consider for other types of engineering applications.

2. Assess if fiber treatments to compatibilize the fiber/matrix interface can improve the mechanical properties of composite bioproducts made with agricultural residue fibers.

The strength, stiffness, and strain behavior of biocomposites produced from PHBV can be changed significantly with the addition of natural fibers, without sacrificing light weight or biodegradability. This improvement in properties can be achieved with the use of high performance industrial fibers, like hemp, as well as fibers recovered from agricultural processes like wheat and barley. The ability to use more common or locally available fibers in biocomposites for 
considerable mechanical improvements may improve their economic viability and sustainability by decreasing the length of the supply chain and the resources needed to grow and harvest fibers for industrial use alone.

3. Evaluate optimal fiber/treatment combinations to enhance mechanical properties (flexural modulus, ultimate flexural strength) for higher performance applications such as automotive panels, aircraft parts, or building construction.

For maximum flexural strength, 30\% grafted hemp composites, $20 \%$ grafted hemp composites and 30\% silanated hemp composites had values that were not statistically distinguishable from each other, but significantly higher than all other samples. The most rigid sample combinations were hemp and hollow stem wheat treated with silane at a weight fraction of $30 \%$. Hemp at $10 \%$ weight fraction treated with silane has high rigidity and strength without a detectable reduction in max strain, making it ideal for applications which require more deformation. Hollow stem wheat performs most similarly to hemp composites in terms of chord modulus, but at a lower flexural strength.

\section{Ongoing studies into these composites should address the following:}

1. Analyze mechanisms of PHBV grafting to determine potential causes for reduced mechanical properties of grafted samples.

The high variability of PHBV grafted samples appears to be influenced by an uneven impact of the treatment process on the matrix of the composite. The effectiveness of the DCP reaction has been shown to be extremely time sensitive [39], and small variations in the mixing and compounding processes may have a negative impact on sample performance. SEM and DSC examination of grafted composites indicated a considerable shift in melting and crystallization temperatures and a visibly altered matrix. 
2. Evaluate alternative methods for low volume high accuracy fiber fraction analysis, or ways to improve the accuracy of the current methods.

Within the resolution of the fiber fraction analysis used in this study, no correlation could be determined between measured fiber fraction and tested mechanical properties. The existing method is sensitive to PHBV residue trapped in the filter.

3. Examine agricultural residue surface chemistry to assess the viability of other fiber treatments.

Each fiber treatment has a different mechanism by which it strengthens the fiber-matrix interface. Because of the variation in surface chemistry and characteristics between different natural fibers, other fiber treatment methods not examined within the scope of this study may be more effective at strengthening agricultural residues and food crop fibers.

4. Test the impact of changing fiber dimensions on flexural strength and modulus of residue composites.

This work focused solely on fibers ground and filtered through a $2 \mathrm{~mm}$ screen and assembled into short chopped fiber composites. Fiber length and diameter in industrial uses will vary considerably, as will the impact of fiber fraction and treatment on fibers of different dimensions. 
REFERENCES 
[1] P. Georgiopoulos, E. Kontou, and A. Christopoulos, "Short-term creep behavior of a biodegradable polymer reinforced with wood-fibers.," Composites: Part B, Engineering, vol. 80, pp. 134-144, oct 2015.

[2] N. Venkatachalam, P. Navaneethakrishnan, R. Rajsekar, and S. Shankar, "Effect of Pretreatment Methods on Properties of Natural Fiber Composites : A Review," vol. 24, no. 7, pp. 555-567, 2016.

[3] A. Shalwan and B. F. Yousif, "In State of Art : Mechanical and tribological behaviour of polymeric composites based on natural fibres," Materials and Design, vol. 48, pp. 14-24, 2013.

[4] R. B. Yusoff, H. Takagi, and A. N. Nakagaito, "Tensile and flexural properties of polylactic acid-based hybrid green composites reinforced by kenaf, bamboo and coir fibers.," Industrial Crops $\&$ Products, vol. 94, pp. 562-573, dec 2016.

[5] O. Faruk, A. K. Bledzki, H.-p. Fink, and M. Sain, "Progress in Polymer Science Biocomposites reinforced with natural fibers : 2000 - 2010," Progress in Polymer Science, vol. 37, no. 11, pp. 1552-1596, 2012.

[6] E. Bioplastics, "PRESS RELEASE: Global bioplastics production capacities continue to grow despite low oil price," p. 2, 2016.

[7] K. H. Rostkowski, C. S. Criddle, and M. D. Lepech, "Cradle-to-gate life cycle assessment for a cradle-to-cradle cycle: Biogas-to-bioplastic (and back)," Environmental Science \& Technology, vol. 46, no. 18, pp. 9822-9829, 2012. PMID: 22775327. 
[8] C. Perez and V. Alvarez, "Ternary composites based on HDPE and MaterBi reinforced with hemp fibres.," Journal of Thermal Analysis \& Calorimetry, vol. 124, pp. 499-508, apr 2016.

[9] M. S. Islam, Z. A. Talib, M. Hasan, I. Ramli, M. K. M. Haafiz, M. Jawaid, A. Islam, and I. M. Inuwa, "Evaluation of mechanical, morphological, and biodegradable properties of hybrid natural fiber polymer nanocomposites.," Polymer Composites, vol. 38, pp. 583-587, mar 2017.

[10] C. A. Ryan, S. L. Billington, and C. S. Criddle, "Biocomposite fiber-matrix treatments that enhance in-service performance can also accelerate end-of-life fragmentation and anaerobic biodegradation to methane," Journal of Polymers and the Environment, vol. 26, no. 4, pp. 1715-1726, 2018.

[11] M. F. V. Marques, J. N. Lunz, V. O. Aguiar, I. Grafova, M. Kemell, and F. Visentin, "Thermal and Mechanical Properties of Sustainable Composites Reinforced with Natural Fibers," Journal of Polymers and the Environment, pp. 251-260, 2015.

[12] S. A. Miller, Application of Time-Dependent Material Properties and Environmental Impact Analysis in Bio-Based Composite Design. PhD thesis, Stanford University, 2014.

[13] S. Panthapulakkal and M. Sain, "Agro-residue reinforced high-density polyethylene composites: Fiber characterization and analysis of composite properties," Composites Part A: Applied Science and Manufacturing, vol. 38, no. 6, pp. 14451454, 2007. 
[14] T. Väisänen, A. Haapala, R. Lappalainen, and L. Tomppo, "Utilization of agricultural and forest industry waste and residues in natural fiber-polymer composites: A review," Waste Management, vol. 54, pp. 62-73, 2016.

[15] C. S. Wortmann, "Harvesting Crop Residues Extension Nutrient Management Specialist; Robert N. Klein, Extension Western Nebraska Crops Specialist; and Charles A. Shapiro, Extension Soil Science - Crop Nutrition Specialist," no. August, 2012.

[16] A. J. Pieja, K. H. Rostkowski, and C. S. Criddle, "Distribution and selection of poly-3-hydroxybutyrate production capacity in methanotrophic proteobacteria.," Microb Ecol, vol. 62, pp. 564-573, oct 2011.

[17] C. A. Ryan, S. L. Billington, and C. S. Criddle, "Assessment of models for anaerobic biodegradation of a model bioplastic: poly(hydroxybutyrate-cohydroxyvalerate)," Bioresource Technology, vol. 227, pp. 205-213, 2017.

[18] N. Venkateshwaran, A. Elaya Perumal, and D. Arunsundaranayagam, "Fiber surface treatment and its effect on mechanical and visco-elastic behaviour of banana/epoxy composite," Materials and Design, vol. 47, pp. 151-159, 2013.

[19] W. V. Srubar III, S. A. Miller, M. D. Lepech, and S. L. Billington, "Incorporating spatiotemporal effects and moisture diffusivity into a multi-criteria materials selection methodology for wood-polymer composites," Construction and Building Materials, vol. 71, pp. 589-601, 2014.

[20] W. V. Srubar III, S. Pilla, Z. C. Wright, C. A. Ryan, J. P. Greene, C. W. Frank, and S. L. Billington, "Mechanisms and impact of fiber-matrix compatibilization techniques on the material characterization of PHBV/oak wood flour engineered 
biobased composites," Composites Science and Technology, vol. 72, no. 6, pp. 708-715, 2012.

[21] B. Arkles, Y. Pan, and Y. M. Kim, "The role of polarity in the structure of silanes employed in surface modification," Silanes and other coupling agents, vol. 5, pp. 51-64, 2009.

[22] M. R. Snowdon, A. K. Mohanty, and M. Misra, "Miscibility and Performance Evaluation of Biocomposites Made from Polypropylene/Poly(lactic acid)/Poly(hydroxybutyrate- co -hydroxyvalerate) with a Sustainable Biocarbon Filler," ACS Omega, vol. 2, pp. 6446-6454, oct 2017.

[23] L. Ruigang, Y. Hui, and H. Yong, "Structure and Morphology of Cellulose in Wheat Straw," Cellulose, vol. 12, no. April 2017, pp. 25-34, 2005.

[24] J. S. Han, "Properties of Nonwood Fibers," Proceedings of the Korean society of wood science, pp. 1-11, 1998.

[25] S. Mani, L. G. Tabil, and S. Sokhansanj, "Effects of compressive force, particle size and moisture content on mechanical properties of biomass pellets from grasses," Biomass and Bioenergy, vol. 30, no. 7, pp. 648-654, 2006.

[26] G. Değer, M. Pakdemirli, F. Candan, S. Akgün, and H. Boyaci, "Strength of wheat and barley stems and design of new beam/columns," Mathematical and Computational Applications, vol. 15, no. 1, pp. 1-13, 2010.

[27] L. Mohammed, M. N. M. Ansari, G. Pua, M. Jawaid, and M. S. Islam, "A Review on Natural Fiber Reinforced Polymer Composite and Its Applications," International Journal of Polymer Science, vol. 2015, pp. 1-15, 2015. 
[28] A. K. Bledzki and A. Jaszkiewicz, "Mechanical performance of biocomposites based on PLA and PHBV reinforced with natural fibres - A comparative study to PP," Composites Science and Technology, vol. 70, no. 12, pp. 1687-1696, 2010.

[29] V. M. Castaño, L. Martínez, and I. Arita, "Preparation and characterization of a natural fiber-polymer resin composite," Materials Letters, vol. 15, no. 1-2, pp. 108-112, 1992.

[30] L. Martino, M. A. Berthet, H. Angellier-Coussy, and N. Gontard, "Understanding external plasticization of melt extruded PHBV-wheat straw fibers biodegradable composites for food packaging," Journal of Applied Polymer Science, vol. 132, no. 10, pp. 1-11, 2015.

[31] S. Modi, K. Cornish, K. Koelling, and Y. Vodovotz, "MECHANICAL AND RHEOLOGICAL PROPERTIES OF PHBV BIOPLASTIC COMPOSITES ENGINEERED WITH INVASIVE PLANT FIBERS.," Transactions of the ASABE, vol. 59, pp. 1883-1891, nov 2016.

[32] Y. K. Jung, T. Y. Kim, S. J. Park, and S. Y. Lee, "Metabolic engineering of Escherichia coli for the production of polylactic acid and its copolymers," Biotechnology and Bioengineering, vol. 105, no. 1, pp. 161-171, 2010.

[33] C. Baley, C. Morvan, and Y. Grohens, "Influence of the Absorbed Water on the Tensile Strength of Flax Fibers," Macromolecular Symposia, no. 222, pp. 195201,2005 .

[34] K. Van De Velde and E. Baetens, "Thermal and mechanical properties of flax fibres as potential composite reinforcement," Macromolecular Materials and Engineering, vol. 286, no. 6, pp. 342-349, 2001. 
[35] T. A. Osswald, L.-S. Turng, and P. Gramann, Injection Molding Handbook. Hanser Gardner Publications, 2nd ed., 2008.

[36] M. Das and D. Chakraborty, "Influence of alkali treatment on the fine structure and morphology of bamboo fibers," Journal of Applied Polymer Science, vol. 102, no. 5, pp. 5050-5056, 2006.

[37] C. A. Ryan, S. L. Billington, and C. S. Criddle, "Methodology to assess endof-life anaerobic biodegradation kinetics and methane production potential for composite materials," Composites Part A: Applied Science and Manufacturing, vol. 95, pp. 388-399, 2017.

[38] A. T. Michel, Characterization and modeling of biobased composites and structural insulated panels. Thesis, Stanford University, 2013.

[39] L. Wei, A. G. Mcdonald, and N. M. Stark, "Grafting of Bacterial Polyhydroxybutyrate (PHB) onto Cellulose via In Situ Reactive Extrusion with Dicumyl Peroxide," Biomacromolecules, vol. 16, no. 3, pp. 1040-1049, 2015.

[40] A. K. Mohanty, M. Misra, and G. Hinrichsen, "Biofibres, biodegradable polymers and biocomposites: An overview," Macromolecular Materials and Engineering, vol. 276-277, no. 1, pp. 1-24, 2000.

[41] A. K. Mohanty, M. Misra, and L. T. Drzal, "Sustainable Bio-Composites from Renewable Resources: Opportunities and Challenges in the Green Materials World," Journal of Polymers and the Environment, vol. 10, no. 1, pp. 19-26, 2002.

[42] J. Knodel, T. Shanower, and P. Beauzay, "Integrated Pest Management of Wheat Stem Sawfly," NDSU Extension Service, vol. 1479, 2016. 
[43] W. V. Srubar, S. Pilla, Z. C. Wright, C. A. Ryan, J. P. Greene, C. W. Frank, and S. L. Billington, "Mechanisms and impact of fiber-matrix compatibilization techniques on the material characterization of PHBV/oak wood flour engineered biobased composites," Composites Science \& Technology, vol. 72, pp. 708-715, mar 2012.

[44] A. T. Michel and S. L. Billington, "Nonlinear Constitutive Model for Anisotropic Biobased Composite Materials.," Journal of Engineering Mechanics, vol. 140, pp. -1 , nov 2014 .

[45] R. Avella, M.; La Rota, G.; Martuscelli, E.; Raimo, M.; Sadocco, P.; Elegir, G.; Riva, "PHBV and wheat straw fibre composites : thermal, mechanical properties and biodegradation," Journal of Materials Science, vol. 5, no. 6, pp. 829-836, 2000.

[46] M. Karakoy, E. Gultepe, S. Pandey, M. A. Khashab, and D. H. Gracias, "Silane surface modification for improved bioadhesion of esophageal stents.," Applied surface science, vol. 311, pp. 684-689, aug 2014.

[47] W. V. Srubar, Z. C. Wright, A. Tsui, A. T. Michel, S. L. Billington, and C. W. Frank, "Characterizing the effects of ambient aging on the mechanical and physical properties of two commercially available bacterial thermoplastics," Polymer Degradation and Stability, vol. 97, no. 10, pp. 1922-1929, 2012.

[48] ASTM, "D 7264 Standard Test Method for Flexural Properties of Polymer Matrix Composite Materials," Astm D7264 / D7264M, vol. i, pp. 1-11, 2015.

[49] O. Shakil, F. Masood, and T. Yasin, "Characterization of physical and biodegradation properties of poly-3-hydroxybutyrate-co-3-hydroxyvalerate/sepiolite 
nanocomposites.," Materials Science \&6 Engineering: C, vol. 77, pp. 173-183, aug 2017.

[50] M. Merrington and C. M. Thompson, "Biometrika Trust Tables of Percentage Points of the Inverted Beta ( F ) Distribution Published by : Oxford University Press on behalf of Biometrika Trust Stable," vol. 33, no. 1, pp. 73-88, 2018.

[51] ASTM, "Standard Test Methods for Constituent Content of Composite Materials," ASTM International, no. May 2009, pp. 1-6, 2010.

[52] M. R. Kessler, Advanced topics in characterization of composites. Trafford Publishing, 2004.

[53] P. J. Barham, A. Keller, E. L. Otun, and P. A. Holmes, "Crystallization and morphology of a bacterial thermoplastic: poly-3-hydroxybutyrate," Journal of Materials Science, vol. 19, no. 9, pp. 2781-2794, 1984.

[54] J. E. Mark, "126. Poly(hydroxybutyrate)," in Polymer Data Handbook (2nd Edition), pp. 741-752, Oxford University Press, 2009.

[55] J. Gu, H. Xu, and C. Wu, "Thermal and crystallization properties of HDPE and HDPE/PP blends modified with DCP," Advances in Polymer Technology, vol. 33, no. 1, pp. 1-5, 2014.

[56] P. Rytlewski, K. Moraczewski, R. Malinowski, and M. Żenkiewicz, "Assessment of dicumyl peroxide ability to improve adhesion between polylactide and flax or hemp fibres.," Composite Interfaces, vol. 21, pp. 671-683, oct 2014.

[57] Y. Xie, C. A. Hill, Z. Xiao, H. Militz, and C. Mai, "Silane coupling agents used for natural fiber/polymer composites: A review," Composites Part A: Applied Science and Manufacturing, vol. 41, no. 7, pp. 806-819, 2010. 
APPENDICES 
$\underline{\text { APPENDIX A }}$

SUPPLEMENTARY MATERIAL 


\section{Detailed Calculations}

The two measures of flexural modulus, chord and secant modulus, were calculated in Matlab using the following process:

Chord modulus is defined by ASTM 7264b as the slope of the stress/strain curve between 2 defined values of strain, with recommended values of 0.001 and 0.003 strain. These strain values are located by Matlab along with the corresponding stress values, at which point the chord modulus is calculated as shown in Equation 3.3.

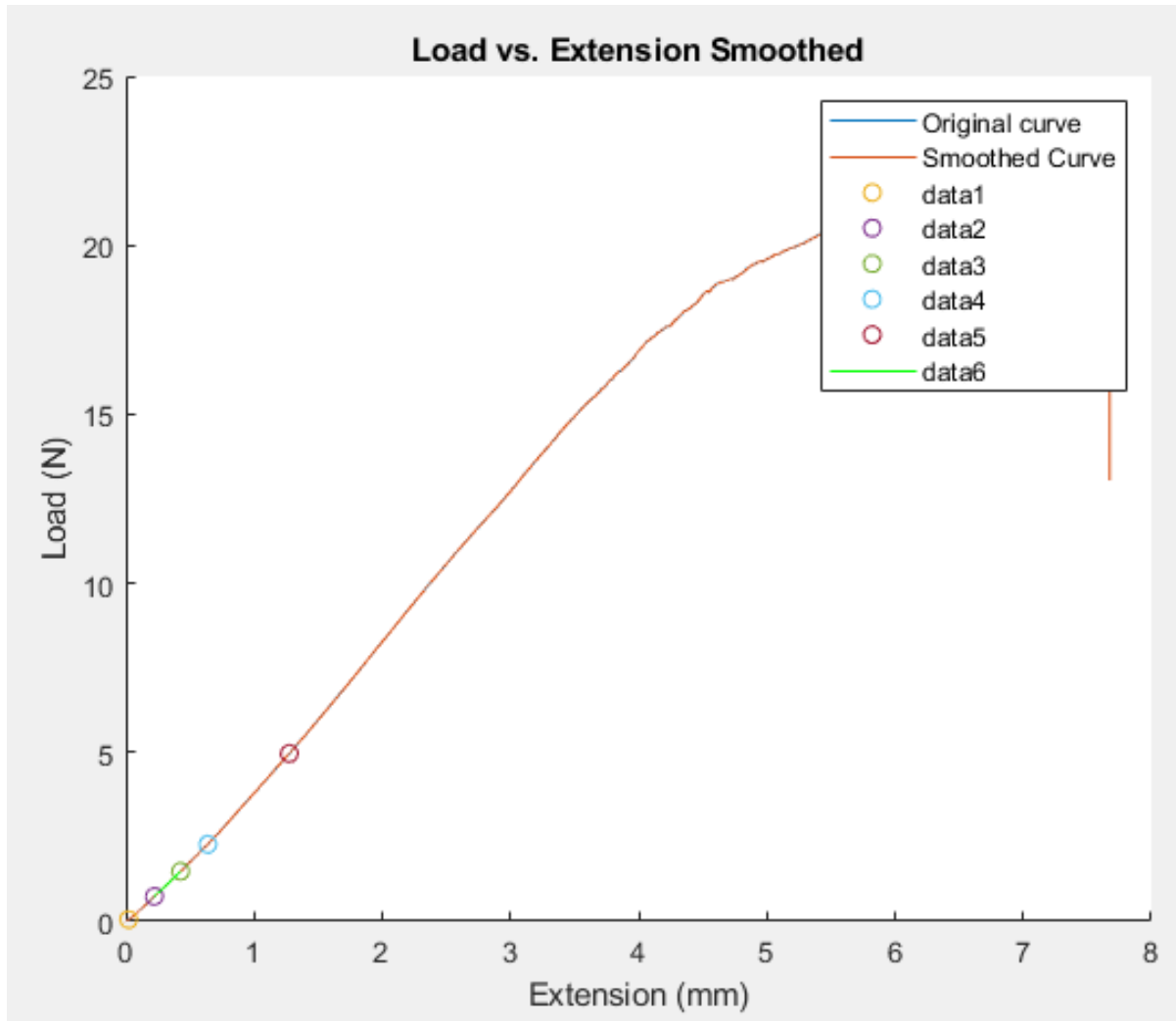

Figure A.1: Initial points of inflection used to calculate secant modulus. The green line between data2 and data3 shows the slope used.

Secant modulus was calculated in a slightly more complex way. It is calculated using the slope of the secant of the force-deflection curve at any given position. 
Because the chord modulus captures flexural behavior after a degree of loading has already been applied, secant modulus was used to measure the initial force deflection behavior of the biocomposites. Matlab was used to locate points of inflection in the initial curve, which may be indicators of different linear regions or changing slope behavior. Once the first points of inflection are located, as seen in Figure A.1, two points can be selected by the user to measure slope. The points selected corresponded to the curve shortly after the initial measurements, which are less stable as the sample settles into position. This slope value replaces $m$ in Equation 3.3, and the secant modulus is then calculated. The full code for all Matlab processes is included in Appendix B.

$\underline{\text { Supplementary Results and Analysis }}$

\section{Mechanical testing:}

Table A.1: Mechanical Properties of Combined Fiber Set

\begin{tabular}{c|ccccc} 
Treatment & Fiber wt.\% & Ultimate Flexural Strength (Mpa) & Strain at UFS & Chord Modulus (Mpa) & Secant Modulus (Mpa) \\
\hline \hline Untreated & 0 & 68.50 & 0.01700 & 5600 & 4750 \\
Untreated & 10 & 66.25 & 0.01600 & 6300 & 6050 \\
Untreated & 20 & 65.75 & 0.01425 & 7500 & 7250 \\
Untreated & 30 & 61.50 & 0.01075 & 8550 & 8250 \\
Silane & 10 & 66.50 & 0.01650 & 6650 & 6450 \\
Silane & 20 & 68.25 & 0.01425 & 8050 & 7800 \\
Silane & 30 & 63.25 & 0.01025 & 9500 & 9200 \\
Grafted & 0 & 63.50 & 0.01650 & 4600 & 4000 \\
Grafted & 10 & 61.00 & 0.01250 & 6100 & 5700 \\
Grafted & 20 & 64.00 & 0.01100 & 7200 & 6750 \\
Grafted & 30 & 66.50 & 0.00950 & 8700 & 8300 \\
\hline
\end{tabular}




\begin{tabular}{|c|c|c|c|c|c|c|c|c|c|c|c|}
\hline $\mathrm{ANO}$ & VA C & DRL & $\mathrm{MO}$ & CON & MPAP & ISON & BY T & MENT & & & \\
\hline & & & & & & & & & & & \\
\hline & $10 \%$ HE & $n$ & 3 & & $20 \%$ HE & $n$ & 3 & & $30 \%$ HE & $n$ & 3 \\
\hline & Untreate & Silanateı & $\mathrm{DCP}$ & & Untreate & Silanate & $\mathrm{DCP}$ & & Untreate & Silanater & $\mathrm{DCP}$ \\
\hline Average & e 6515 & 6979 & 6290 & Average & 7729 & 8313.1 & 7153 & Average & 8418 & 10157 & 8895 \\
\hline STDeV & 111 & 297 & 521 & STDeV & 147 & 117.68 & 317 & STDeV & 161 & 202 & 328 \\
\hline & all 3 & unttsil & untfdep & & all 3 & unttsil & untfdep & & all 3 & unttsil & untedcp \\
\hline$s 2$ & 42275 & 17342 & 84307 & $s 2$ & 11627 & 438.13 & 14483 & $s 2$ & 7600.4 & 869.97 & 14023 \\
\hline Var_Ave & $\in 123534$ & 107741 & 25325 & Var_Ave & 336214 & 170379 & 165845 & Var_Ave & E 807046 & $2 E+06$ & 113612 \\
\hline & $95 \%$ & $99 \%$ & $<75 \%$ & & $99.90 \%$ & $99.90 \%$ & $99 \%$ & & $99.90 \%$ & $99.90 \%$ & $99 \%$ \\
\hline $\mathrm{F}$ & 8.7665 & 18.638 & 0.9012 & $\mathrm{~F}$ & 86.752 & 1166.6 & 34.353 & $\mathrm{~F}$ & 318.55 & 5211.6 & 24.305 \\
\hline$f 1$ & 2 & dof - 1 in & material & f1 & 2 & dof -1 in & material & f1 & 2 & dof - 1 in $n$ & material \\
\hline 12 & 4 & dof mea: & surement & \&2 & 4 & dof meas & surement & 12 & 4 & dof meas & surement \\
\hline & & & & & & & & & & & \\
\hline & $10 \% \mathrm{BA}$ & $n$ & 3 & & $20 \% \mathrm{BA}$ & $n$ & 3 & & $30 \% \mathrm{BA}$ & $n$ & 3 \\
\hline & Untreate & Silanater & DCP & & Untreate & Silanater & DCP & & Untreate & Silanater & DCP \\
\hline Average & e 6029 & 6432 & 6299 & Average & 7399 & 7331 & 7272 & Average & 8321 & 8936 & 9135 \\
\hline STDeV & 538 & 265 & 362 & STDeV & 277 & 41 & 312 & STDeV & 485 & 706 & 356 \\
\hline & all 3 & unttsil & untidep & & all 3 & unttsil & untidep & & all 3 & unttsil & untidep \\
\hline$s 2$ & 19195 & 37356 & 15500 & $s 2$ & 21736 & 27868 & 610.56 & $s 2$ & 31242 & 24377 & 8280 \\
\hline Var_Ave & E $\quad 42141$ & 81098 & 36576 & Var_Ave & 4041.6 & 2323.3 & 8068.7 & Var_Ave & E 179937 & 188820 & 331135 \\
\hline & $90.00 \%$ & $90.00 \%$ & $95 \%$ & & $<75 \%$ & $<75 \%$ & $99 \%$ & & $95.00 \%$ & $99.00 \%$ & $99.9 \%$ \\
\hline $\mathrm{F}$ & 6.5861 & 6.5129 & 7.0794 & $\mathrm{~F}$ & 0.5578 & 0.2501 & 39.646 & $\mathrm{~F}$ & 17.278 & 23.238 & 119.98 \\
\hline 11 & 2 & dof - 1 in & material & f1 & 2 & dof - 1 in & material & f1 & 2 & dof - 1 in $n$ & material \\
\hline 12 & 4 & dof meas & surement & 12 & 4 & dof meas & surement & 12 & 4 & dof meas & surement \\
\hline & & & & & & & & & & & \\
\hline & $10 \% 55$ & $n$ & 3 & & $20 \% \mathrm{SS}$ & $n$ & 3 & & $30 \%$ SS & $n$ & 3 \\
\hline & Untreate & Silanate! & DCP & & Untreate & Silanater & $\mathrm{DCP}$ & & Untreate & Silanater & DCP \\
\hline Average & 6222 & 6601 & 5726 & Average & 7421 & 8098 & 7080 & Average & 8937 & 9461 & 8585 \\
\hline STDeV & 230 & 270 & 375 & STDeV & 150 & 176 & 274 & STDeV & 143 & 271 & 298 \\
\hline & all 3 & unttsil & untidep & & all 3 & unttsil & untidep & & all 3 & unttsil & untidep \\
\hline$s 2$ & 5596 & 783.41 & 10475 & $s 2$ & 4273 & 332.4 & 7674.2 & $s 2$ & 6816.1 & 8176.2 & 11920 \\
\hline Var_Ave & E 192560 & 71915 & 122909 & Var_Ave & 268836 & 229481 & 58203 & Var_Ave & 194413 & 137288 & 62041 \\
\hline & $99.90 \%$ & $99.90 \%$ & $99 \%$ & & $99.90 \%$ & $99.90 \%$ & $99 \%$ & & $99.90 \%$ & $99.00 \%$ & $95 \%$ \\
\hline $\mathrm{F}$ & 103.23 & 275.39 & 35.2 & $\mathrm{~F}$ & 188.75 & 2071.1 & 22.753 & $\mathrm{~F}$ & 85.567 & 50.373 & 15.614 \\
\hline f1 & 2 & dof - 1 in & material & f1 & 2 & dof - 1 in & material & f1 & 2 & dof - 1 in $n$ & material \\
\hline 12 & 4 & dof mea: & surement & 12 & 4 & dof meas & surement & 12 & 4 & dof meas & surement \\
\hline & & & & & & & & & & & \\
\hline & $10 \% \mathrm{HS}$ & $n$ & 3 & & $20 \% \mathrm{HS}$ & $n$ & 3 & & $30 \%$ HS & $n$ & 3 \\
\hline & Untreate & Silanate! & $\mathrm{DCP}$ & & Untreate & Silanate & $\mathrm{DCP}$ & & Untreate & Silanater & $\mathrm{DCP}$ \\
\hline Average & e $\quad 6317$ & 6575 & 6332 & Average & 7607 & 8129 & 7318 & Average & 8577 & 9879 & 8451 \\
\hline STDeV & 190 & 355 & 310 & STDeV & 198 & 298 & 191 & STDeV & 442 & 381 & 523 \\
\hline & all 3 & unttsil & untidep & & all 3 & unttsil & unttdep & & all 3 & unttsil & untidep \\
\hline$s 2$ & 7276 & 13606 & 7218.7 & $s 2$ & 3571.5 & 5024.5 & 19.526 & $s 2$ & 5053.6 & 1856.3 & 3262.9 \\
\hline Var_Ave & 20998 & 33299 & 109.82 & Var_Ave & 168828 & 136299 & 41623 & Var_Ave & 625052 & 846984 & 8045.5 \\
\hline & $95.0 \%$ & $95.0 \%$ & $<75 \%$ & & $99.9 \%$ & $99.9 \%$ & $99.9 \%$ & & $99.9 \%$ & $99.9 \%$ & $95.0 \%$ \\
\hline $\mathrm{F}$ & 8.6577 & 7.3424 & 0.0456 & $\mathrm{~F}$ & 141.81 & 81.38 & 6394.9 & $\mathrm{~F}$ & 371.06 & 1368.8 & 7.3971 \\
\hline f1 & 2 & dof - 1 in & material & f1 & 2 & dof - 1 in & material & f1 & 2 & dof - 1 in $n$ & material \\
\hline 12 & 4 & dof mea: & surement & 12 & 4 & dof meas & surement & 12 & 4 & dof meas & surement \\
\hline
\end{tabular}

Figure A.2: $\mathrm{F}$ test analysis of chord modulus of each fiber and fiber fraction by treatment. Green is significant with $99 \%$ confidence or greater, while red is not a statistically significant result. 


\begin{tabular}{|c|c|c|c|c|c|c|c|c|c|c|c|c|c|c|}
\hline \multicolumn{15}{|c|}{ ANOVA CHORD MODULUS COMPARISON BY FIBER } \\
\hline & & & & & & & & & & & & & & \\
\hline & \multicolumn{2}{|c|}{$10 \%$ Untreated } & n & 3 & & $20 \%$ Un & treated & & 3 & & \multicolumn{3}{|c|}{ 30\% Untreated n } & 3 \\
\hline & Hemp & Barley & SS Wheat & HS Wheat & & Hemp & Barley & \multicolumn{2}{|c|}{ SS Whe: HS Wheat } & & Hemp & Barley & \multicolumn{2}{|c|}{ SS Whe: HS Wheat } \\
\hline Average & 6515 & 6029 & 6222 & 6317 & Average & 7729 & 7399 & 7421 & 7607 & Average & 8418 & 8321 & 8937 & 8577 \\
\hline \multirow{2}{*}{ STDeV } & \multicolumn{2}{|r|}{538} & 230 & 190 & STDeV & 147 & 277 & 150 & 198 & STDeV & \multicolumn{2}{|r|}{485} & 143 & 442 \\
\hline & all 4 & helba & hedss & heths & & all 4 & herba & hedss & heths & & all 4 & herba & hetss & heths \\
\hline$s 2$ & 35050 & 91306.695 & 7163.485 & 3147.6643 & $s 2$ & 3699.9 & 8467 & \multirow{2}{*}{\begin{tabular}{|r|}
3.2447 \\
47563
\end{tabular}} & 1261.4 & $s 2$ & 32706 & 52597 & 145.88 & 39672 \\
\hline \multirow[t]{2}{*}{ Var_Ave } & 40871 & 118096.38 & 42830.79 & 19538.691 & Var_Ave & 24815 & 54651 & & 7509.3 & Var_Ave & 73202 & 4721 & \multirow{2}{*}{$\begin{array}{r}134584 \\
99.90 \%\end{array}$} & 12649 \\
\hline & & & 95 & 99 & & $99 \%$ & $99 \%$ & $99.90 \%$ & 95 & & & & & \multirow[b]{2}{*}{0.9565} \\
\hline $\mathrm{F}$ & 3.4982 & 3.8802099 & 17.93713 & 18.622086 & $\mathrm{~F}$ & 20.121 & 19.364 & 43976 & 17.859 & $\mathrm{~F}$ & 6.7145 & 0.2693 & 2767.6 & \\
\hline 11 & 2 & \multicolumn{2}{|c|}{ dof -1 in material } & & f1 & & dof -1 in $r$ & material & & f1 & 2 & dof - 1 in $r$ & material & \\
\hline$\$ 2$ & 4 & dof measure & rement & & $\$ 2$ & 4 & dof meas & surement & & 12 & 4 & dof meas & surement & \\
\hline & & & & & & & & & & & & & & \\
\hline & $10 \%$ Sil & lane & $n$ & 3 & & $20 \%$ sil & lane & $n$ & 3 & & $30 \%$ sil & lane & $n$ & 3 \\
\hline & Hemp & Barley & SS Wheat & HS Wheat & & Hemp & Barley & SS Whe: & HS Wheat & & Hemp & Barley & SS Whe: & : HS Wheat \\
\hline Average & 6979 & 6432 & 6601 & 6575 & Average & 8313 & 7331 & 8098 & 8129 & Average & $=10157$ & 8936 & 9461 & 9879 \\
\hline STDeV & 297 & 265 & 270 & 355 & STDeV & 118 & 41 & 176 & 298 & STDeV & 202 & 706 & 271 & 381 \\
\hline & all 4 & herba & hetss & heths & & all 4 & herba & hedss & heths & & all 4 & herba & hedss & herths \\
\hline$s 2$ & 1712.4 & 519.59998 & 363.3307 & 1685.8509 & $s 2$ & 11693 & 2915 & 1678.1 & 16214 & $s 2$ & 49686 & 126718 & 2386.2 & 16027 \\
\hline Var_Ave & 54629 & 149856.23 & 71302.21 & 81532.604 & Var_Ave & $=189431$ & 482673 & 23049 & 16962 & Var_Ave & $=282756$ & 745695 & 242046 & 38642 \\
\hline & $99.9 \%$ & $99.9 \%$ & $99.9 \%$ & $99.9 \%$ & & $99.0 \%$ & $99.9 \%$ & $99.0 \%$ & & & $95 \%$ & $95 \%$ & 99.9 & 95 \\
\hline $\mathrm{F}$ & 95.705 & 865.22073 & 588.738 & 145.08863 & $\mathrm{~F}$ & 48.6 & 496.75 & 41.205 & 3.1386 & $\mathrm{~F}$ & 17.073 & 17.654 & 304.3 & 7.2331 \\
\hline f1 & 2 & dof - 1 in ma & terial & & f1 & 2 & dof -1 in $r$ & material & & f1 & 2 & dof -1 in $r$ & material & \\
\hline 12 & 4 & dof measur & rement & & 12 & 4 & dof meas & surement & & f2 & 4 & dof meas & surement & \\
\hline & & & & & & & & & & & & & & \\
\hline & $10 \%$ dep & & $\mathrm{n}$ & 3 & & $20 \%$ dep & & $n$ & 3 & & $30 \%$ de & & $n$ & 3 \\
\hline & Hemp & Barley & SS Wheat & HS Wheat & & Hemp & Barley & SS Whe: & HS Wheat & & Hemp & Barley & SS Whe: & : HS Whea \\
\hline Average & 6290 & 6299 & 5726 & 6332 & Average & 7153 & 7272 & 7080 & 7318 & Average & 8895 & 9135 & 8585 & 8451 \\
\hline STDeV & 521 & 362 & 375 & 310 & STDeV & 317 & 312 & 274 & 191 & STDeV & 328 & 356 & 298 & 523 \\
\hline & all 4 & helba & hetss & heths & & all 4 & herba & hedss & heths & & all 4 & herba & herlss & heths \\
\hline$s 2$ & 8200.9 & 12697.632 & 10685.35 & 22287.207 & $s 2$ & 3402.3 & 13.098 & 957.33 & 7965 & $s 2$ & 10137 & 396.99 & 454.48 & 19011 \\
\hline Var_Ave & 84619 & 45.362812 & 158723.9 & 890.20901 & Var_Ave & 11876 & 6995.5 & 2714.6 & 13588 & Var_Ave & 95031 & 28788 & 48087 & 98779 \\
\hline & $99 \%$ & & 99 & & & $95 \%$ & $99.9 \%$ & $95 \%$ & & & $99 \%$ & $99.9 \%$ & $99.9 \%$ & \\
\hline $\mathrm{F}$ & 30.955 & 0.0107176 & 44.56304 & 0.1198278 & $\mathrm{~F}$ & 10.472 & 1602.3 & 8.5069 & 5.1178 & $\mathrm{~F}$ & 28.125 & 217.55 & 317.42 & 15.588 \\
\hline f1 & 2 & dof - 1 in mat & aterial & & f1 & 2 & dof - 1 in & material & & f1 & & dof - 1 in & material & \\
\hline \&2 & 4 & dof measure & rement & & f2 & 4 & dof meas & surement & & f2 & 4 & dof meas & surement & \\
\hline
\end{tabular}

Figure A.3: $\mathrm{F}$ test analysis of chord modulus of each treatment and fiber fraction by fiber. Green is significant with $99 \%$ confidence or greater, while red is not a statistically significant result. 


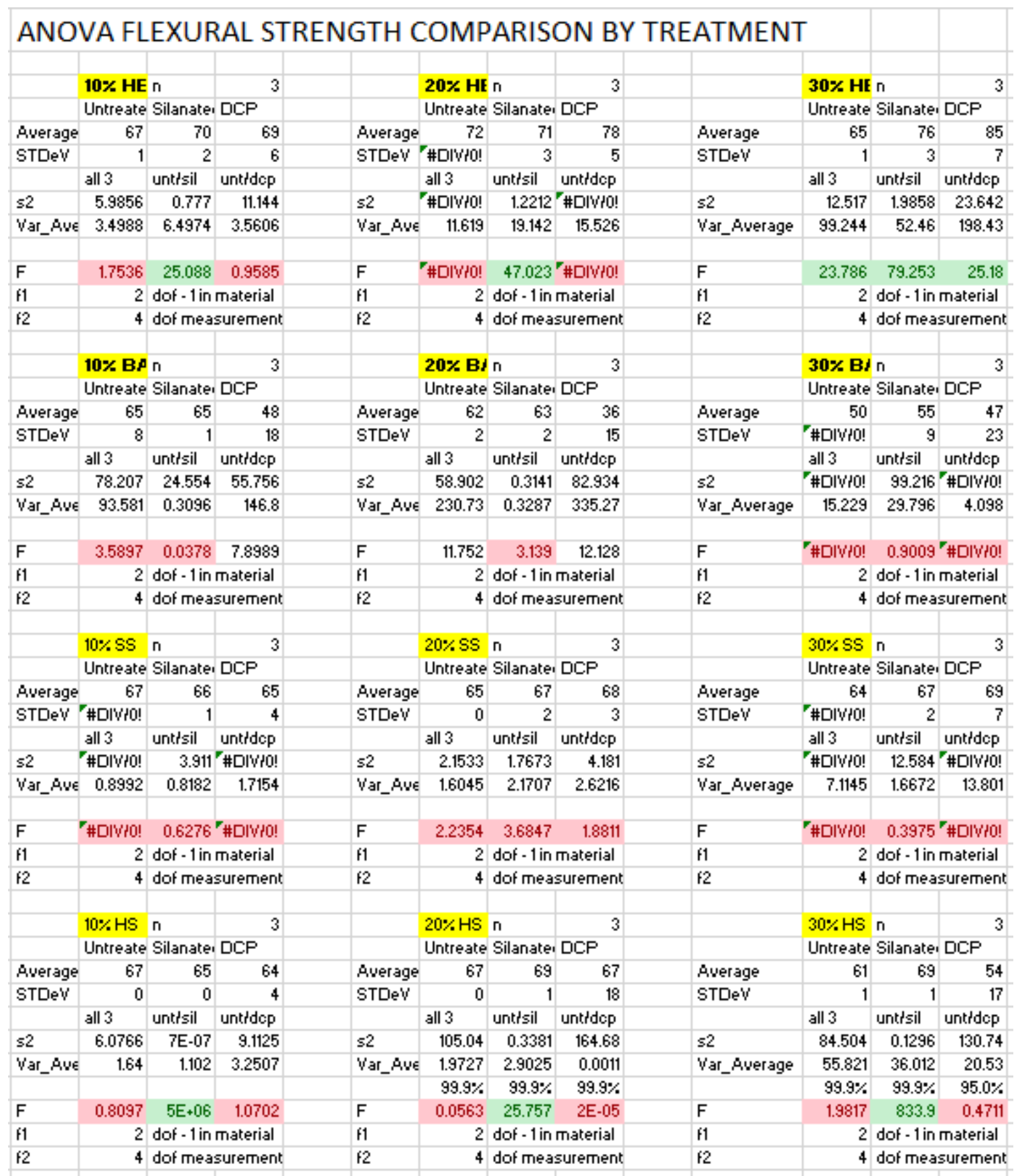

Figure A.4: $\mathrm{F}$ test analysis of flexural strength of each fiber and fiber fraction by treatment. Green is significant with $99 \%$ confidence or greater, while red is not a statistically significant result. 


\begin{tabular}{|c|c|c|c|c|c|c|c|c|c|c|c|c|c|c|}
\hline \multicolumn{15}{|c|}{ ANOVA FLEXURAL STRENGTH COMPARISON BY FIBER } \\
\hline & & & & & & \multirow{2}{*}{\multicolumn{3}{|c|}{$20 \%$ Untreated $n$}} & & & & & & \\
\hline & \multicolumn{2}{|c|}{$10 \%$ Untreated } & $\mathrm{n}$ & 3 & & & & & 3 & & \multicolumn{3}{|c|}{$30 \%$ Untreated $n$} & 3 \\
\hline & Hemp & Barley & \multicolumn{2}{|c|}{ SS Whe: HS Wheat } & & Hemp & Barley & \multicolumn{2}{|c|}{ SS Whe: HS Wheat } & & Hemp & Barley & \multicolumn{2}{|c|}{$\begin{array}{ll}n & 3 \\
S S & \text { whe: HS Wheat }\end{array}$} \\
\hline \multirow{3}{*}{$\begin{array}{l}\text { Average } \\
\text { STDeV }\end{array}$} & 67 & 65 & 67 & 67 & \multicolumn{2}{|c|}{ Average $\quad 72$} & 62 & 65 & 67 & Average & 65 & 50 & \multicolumn{2}{|c|}{$64 \quad 61$} \\
\hline & \multicolumn{2}{|c|}{$1 \quad 8$} & \#DIV10! & 0 & \multicolumn{2}{|c|}{ STDeV '\#DIVHo! } & 2 & 0 & 0 & STDeV & \multicolumn{2}{|c|}{ 1"\#DIV/o! } & \multicolumn{2}{|l|}{ \#DIViro! } \\
\hline & all 4 & herba & heitss & heths & 3 & all 4 & helba & hetss $\mathrm{r}$ & heths & & all 4 & herlba & heiss & heths \\
\hline$s 2$ & 17.191 & 21.905 & \#DIVro! & 0.5169 & $s 2$ & \#DIV10! & 1.8176 & 2.9469 & 2.4865 & $s 2$ & \#DIvio! & \#DIvio! & \#DIVro! & 0.0002 \\
\hline Var_Ave & 0.5588 & 0.5694 & 0.0042 & 0.0697 & Var_Ave & 17.545 & 5.8175 & 5.3394 & 11.078 & Var_Ave & 47.574 & 116.49 & 1.0129 & 11.127 \\
\hline $\mathrm{F}$ & 0.0975 & $0.078^{\prime}$ & \#DIVio! & 0.4045 & $\mathrm{~F}$ & \#DIVio! & 9.6019 & 5.4357 & 13.366 & $\mathrm{~F}$ & \#DIVн! & "\#DIVн! & \#DIVro! & 221202 \\
\hline f1 & \multicolumn{3}{|c|}{2 dof - 1 in material } & & f1 & 2 & dof - 1 in $r$ & material & & f1 & 2 & dof - 1 in $n$ & material & \\
\hline \multirow[t]{4}{*}{12} & 4 & dof meas & surement & & ^2 & 4 & dof meas & surement & & 12 & 4 & dof meas & surement & \\
\hline & & & & & & & & & & & & & & \\
\hline & $10 \%$ Sil & lane & $n$ & 3 & & $20 \%$ sil & lane & $\mathrm{n}$ & 3 & & $30 \%$ sil & lane & $n$ & 3 \\
\hline & Hemp & Barley & SS whe: & HS Wheat & & Hemp & Barley & SS Whe: & HS Wheat & & Hemp & Barley & SS Whe: & HS Wheat \\
\hline Average & 70 & 65 & 66 & 65 & Average & 71 & 63 & 67 & 69 & Average & 76 & 55 & 67 & 69 \\
\hline STDeV & 2 & 1 & 1 & 0 & STDeV & 3 & 2 & 2 & 1 & STDeV & 3 & 9 & 2 & 1 \\
\hline & all 4 & herba & heitss & heths & & all 4 & helba & hetss & herths & & all 4 & herlba & hetss & herths \\
\hline$s 2$ & 0.9117 & 1.3372 & 0.7705 & 2.5639 & $s 2$ & 0.6341 & 0.839 & 0.5516 & 1.8249 & $s 2$ & 12.169 & 19.105 & 0.1839 & 1.0753 \\
\hline Var_Ave & 5.9641 & 14.9 & 8.348 & 11.121 & Var_Ave & 13.332 & 37.205 & 8.3457 & 2.6927 & Var_Ave & 74.528 & 213.21 & 33.937 & 20.955 \\
\hline & $99.9 \%$ & $99.9 \%$ & $99.9 \%$ & $99.9 \%$ & & $99.0 \%$ & $99.9 \%$ & $99.0 \%$ & & & $95 \%$ & $95 \%$ & 99.9 & 95 \\
\hline $\mathrm{F}$ & 19.624 & 33.428 & 32.502 & 13.012 & $\mathrm{~F}$ & 63.073 & 133.04 & 45.386 & 4.4265 & $\mathrm{~F}$ & 18.372 & 33.48 & 553.75 & 58.462 \\
\hline$f 1$ & 2 & dof -1 in $n$ & material & & f1 & 2 & dof - 1 in $n$ & material & & f1 & 2 & dof - 1 in $n$ & material & \\
\hline 12 & 4 & dof meas & surement & & 12 & 4 & dof meas & surement & & \$2 & 4 & dof meas & surement & \\
\hline & & & & & & & & & & & & & & \\
\hline & $10 \%$ dep & & $\mathrm{n}$ & 3 & & $20 \%$ dc & & $n$ & 3 & & $30 \%$ dep & & $n$ & 3 \\
\hline & Hemp & Barley & SS Whe: & HS Wheat & & Hemp & Barley & SS Whe: & HS Wheat & & Hemp & Barley & SS Whe: & HS Wheat \\
\hline Average & 69 & 48 & 65 & 64 & Average & 78 & 36 & 68 & 67 & Average & 85 & 47 & 69 & 54 \\
\hline STDeV & 6 & 18 & 4 & 4 & STDeV & 5 & 15 & 3 & 18 & STDeV & 7 & 23 & 7 & 17 \\
\hline & all 4 & herba & heitss & heths & & all 4 & helba & hetss & heths & & all 4 & herlba & heitss & heths \\
\hline$s 2$ & 46.824 & 77.599 & 1.8414 & 1.0786 & $s 2$ & 59.466 & 58.465 & 1.2825 & 95.948 & $s 2$ & 58.975 & 118.34 & 0.1119 & 43.352 \\
\hline Var_Ave & 83.833 & 217.79 & 9.8079 & 11.737 & Var_Ave & 322.42 & 861.92 & 50.67 & 60.099 & Var_Ave & 285.44 & 723.83 & 129.46 & 481.95 \\
\hline & $99 \%$ & & 99 & & & $95 \%$ & $99.9 \%$ & $95 \%$ & & & $99 \%$ & $99.9 \%$ & $99.9 \%$ & \\
\hline$F$ & 5.3712 & 8.4199 & 15.979 & 32.646 & $\mathrm{~F}$ & 16.266 & 44.228 & 118.52 & 1.8791 & $\mathrm{~F}$ & 14.52 & 18.349 & 3470.1 & 33.351 \\
\hline$f 1$ & 2 & dof -1 in $n$ & material & & f1 & 2 & dof - 1 in $n$ & material & & f1 & 2 & dof - 1 in $n$ & material & \\
\hline 12 & 4 & dof meas & surement & & 12 & 4 & dof meas & surement & & 12 & 4 & dof meas & surement & \\
\hline
\end{tabular}

Figure A.5: $\mathrm{F}$ test analysis flexural strength of each treatment and fiber fraction by fiber. Green is significant with $99 \%$ confidence or greater, while red is not a statistically significant result. 


\begin{tabular}{|c|c|c|c|c|c|c|c|c|c|c|c|c|c|c|}
\hline \multicolumn{15}{|c|}{ ANOVA FLEXURAL STRENGTH COMPARISON BY FIBER } \\
\hline & & & & & & \multirow{2}{*}{\multicolumn{3}{|c|}{$20 \%$ Untreated $n$}} & & & & & & \\
\hline & \multicolumn{2}{|c|}{$10 \%$ Untreated } & $\mathrm{n}$ & 3 & & & & & 3 & & \multicolumn{3}{|c|}{$30 \%$ Untreated $n$} & 3 \\
\hline & Hemp & Barley & \multicolumn{2}{|c|}{ SS Whe: HS Wheat } & & Hemp & Barley & \multicolumn{2}{|c|}{ SS Whe: HS Wheat } & & Hemp & Barley & \multicolumn{2}{|c|}{$\begin{array}{ll}n & 3 \\
S S & \text { whe: HS Wheat }\end{array}$} \\
\hline \multirow{3}{*}{$\begin{array}{l}\text { Average } \\
\text { STDeV }\end{array}$} & 67 & 65 & 67 & 67 & \multicolumn{2}{|c|}{ Average $\quad 72$} & 62 & 65 & 67 & Average & 65 & 50 & \multicolumn{2}{|c|}{$64 \quad 61$} \\
\hline & \multicolumn{2}{|c|}{$1 \quad 8$} & \#DIV10! & 0 & \multicolumn{2}{|c|}{ STDeV '\#DIVHo! } & 2 & 0 & 0 & STDeV & \multicolumn{2}{|c|}{ 1"\#DIV/o! } & \multicolumn{2}{|l|}{ \#DIViro! } \\
\hline & all 4 & herba & heitss & heths & 3 & all 4 & helba & hetss $\mathrm{r}$ & heths & & all 4 & herlba & heiss & heths \\
\hline$s 2$ & 17.191 & 21.905 & \#DIVro! & 0.5169 & $s 2$ & \#DIV10! & 1.8176 & 2.9469 & 2.4865 & $s 2$ & \#DIvio! & \#DIvio! & \#DIVro! & 0.0002 \\
\hline Var_Ave & 0.5588 & 0.5694 & 0.0042 & 0.0697 & Var_Ave & 17.545 & 5.8175 & 5.3394 & 11.078 & Var_Ave & 47.574 & 116.49 & 1.0129 & 11.127 \\
\hline $\mathrm{F}$ & 0.0975 & $0.078^{\prime}$ & \#DIVio! & 0.4045 & $\mathrm{~F}$ & \#DIVio! & 9.6019 & 5.4357 & 13.366 & $\mathrm{~F}$ & \#DIVн! & "\#DIVн! & \#DIVro! & 221202 \\
\hline f1 & \multicolumn{3}{|c|}{2 dof - 1 in material } & & f1 & 2 & dof - 1 in $r$ & material & & f1 & 2 & dof - 1 in $n$ & material & \\
\hline \multirow[t]{4}{*}{12} & 4 & dof meas & surement & & ^2 & 4 & dof meas & surement & & 12 & 4 & dof meas & surement & \\
\hline & & & & & & & & & & & & & & \\
\hline & $10 \%$ Sil & lane & $n$ & 3 & & $20 \%$ sil & lane & $\mathrm{n}$ & 3 & & $30 \%$ sil & lane & $n$ & 3 \\
\hline & Hemp & Barley & SS whe: & HS Wheat & & Hemp & Barley & SS Whe: & HS Wheat & & Hemp & Barley & SS Whe: & HS Wheat \\
\hline Average & 70 & 65 & 66 & 65 & Average & 71 & 63 & 67 & 69 & Average & 76 & 55 & 67 & 69 \\
\hline STDeV & 2 & 1 & 1 & 0 & STDeV & 3 & 2 & 2 & 1 & STDeV & 3 & 9 & 2 & 1 \\
\hline & all 4 & herba & heitss & heths & & all 4 & helba & hetss & herths & & all 4 & herlba & hetss & herths \\
\hline$s 2$ & 0.9117 & 1.3372 & 0.7705 & 2.5639 & $s 2$ & 0.6341 & 0.839 & 0.5516 & 1.8249 & $s 2$ & 12.169 & 19.105 & 0.1839 & 1.0753 \\
\hline Var_Ave & 5.9641 & 14.9 & 8.348 & 11.121 & Var_Ave & 13.332 & 37.205 & 8.3457 & 2.6927 & Var_Ave & 74.528 & 213.21 & 33.937 & 20.955 \\
\hline & $99.9 \%$ & $99.9 \%$ & $99.9 \%$ & $99.9 \%$ & & $99.0 \%$ & $99.9 \%$ & $99.0 \%$ & & & $95 \%$ & $95 \%$ & 99.9 & 95 \\
\hline $\mathrm{F}$ & 19.624 & 33.428 & 32.502 & 13.012 & $\mathrm{~F}$ & 63.073 & 133.04 & 45.386 & 4.4265 & $\mathrm{~F}$ & 18.372 & 33.48 & 553.75 & 58.462 \\
\hline$f 1$ & 2 & dof -1 in $n$ & material & & f1 & 2 & dof - 1 in $n$ & material & & f1 & 2 & dof - 1 in $n$ & material & \\
\hline 12 & 4 & dof meas & surement & & 12 & 4 & dof meas & surement & & \$2 & 4 & dof meas & surement & \\
\hline & & & & & & & & & & & & & & \\
\hline & $10 \%$ dep & & $\mathrm{n}$ & 3 & & $20 \%$ dc & & $n$ & 3 & & $30 \%$ dep & & $n$ & 3 \\
\hline & Hemp & Barley & SS Whe: & HS Wheat & & Hemp & Barley & SS Whe: & HS Wheat & & Hemp & Barley & SS Whe: & HS Wheat \\
\hline Average & 69 & 48 & 65 & 64 & Average & 78 & 36 & 68 & 67 & Average & 85 & 47 & 69 & 54 \\
\hline STDeV & 6 & 18 & 4 & 4 & STDeV & 5 & 15 & 3 & 18 & STDeV & 7 & 23 & 7 & 17 \\
\hline & all 4 & herba & heitss & heths & & all 4 & helba & hetss & heths & & all 4 & herlba & heitss & heths \\
\hline$s 2$ & 46.824 & 77.599 & 1.8414 & 1.0786 & $s 2$ & 59.466 & 58.465 & 1.2825 & 95.948 & $s 2$ & 58.975 & 118.34 & 0.1119 & 43.352 \\
\hline Var_Ave & 83.833 & 217.79 & 9.8079 & 11.737 & Var_Ave & 322.42 & 861.92 & 50.67 & 60.099 & Var_Ave & 285.44 & 723.83 & 129.46 & 481.95 \\
\hline & $99 \%$ & & 99 & & & $95 \%$ & $99.9 \%$ & $95 \%$ & & & $99 \%$ & $99.9 \%$ & $99.9 \%$ & \\
\hline$F$ & 5.3712 & 8.4199 & 15.979 & 32.646 & $\mathrm{~F}$ & 16.266 & 44.228 & 118.52 & 1.8791 & $\mathrm{~F}$ & 14.52 & 18.349 & 3470.1 & 33.351 \\
\hline$f 1$ & 2 & dof -1 in $n$ & material & & f1 & 2 & dof - 1 in $n$ & material & & f1 & 2 & dof - 1 in $n$ & material & \\
\hline 12 & 4 & dof meas & surement & & 12 & 4 & dof meas & surement & & 12 & 4 & dof meas & surement & \\
\hline
\end{tabular}

Figure A.6: $\mathrm{F}$ test analysis flexural strength of each treatment and fiber fraction by fiber. Green is significant with $99 \%$ confidence or greater, while red is not a statistically significant result. 


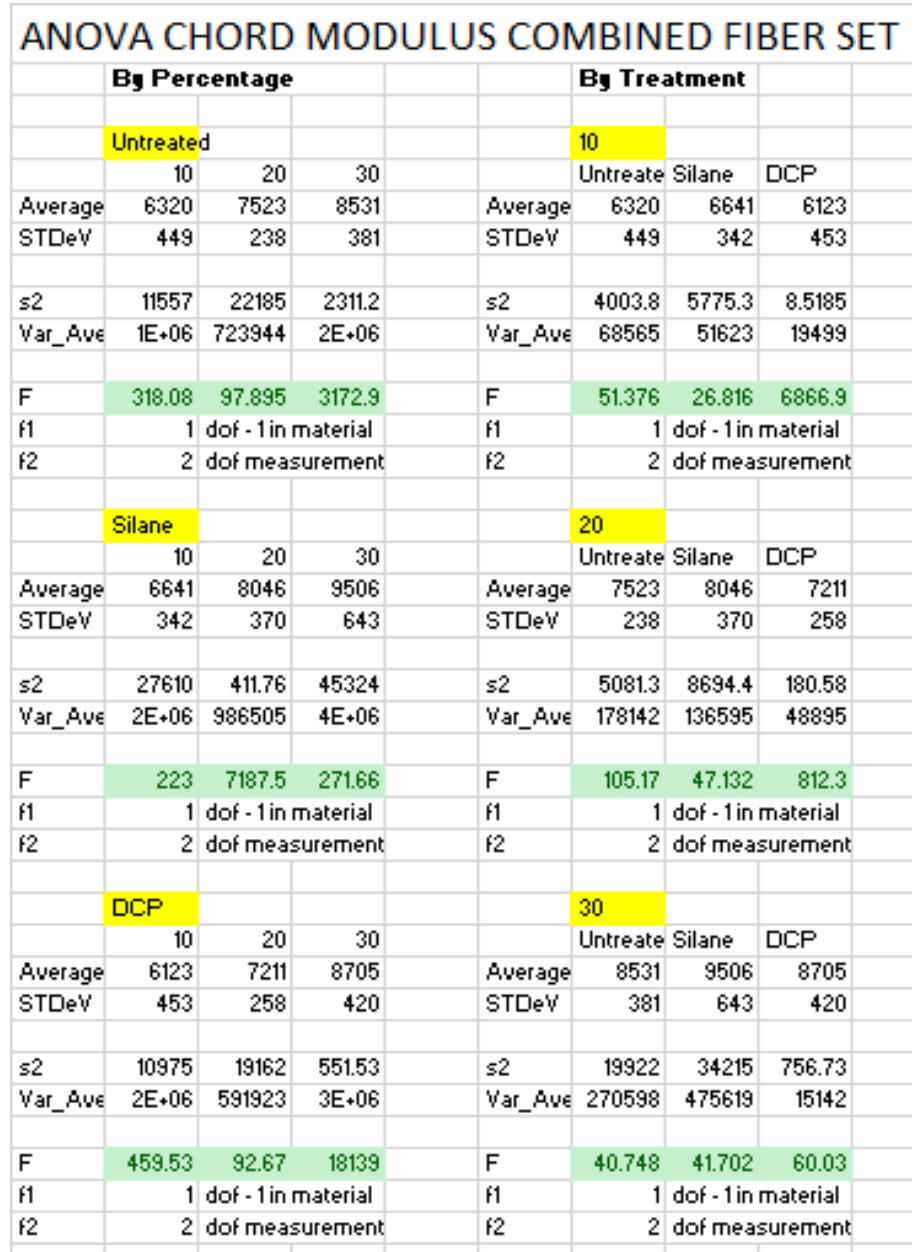

Figure A.7: $\mathrm{F}$ test analysis flexural strength of each treatment and fiber fraction by fiber. Green is significant with $99 \%$ confidence or greater, while red is not a statistically significant result. 


\begin{tabular}{|c|c|c|c|c|c|c|c|}
\hline $\mathrm{ANO}$ & VA FL & EXUR & $\mathrm{ALS}$ & 3TH C & COMB & INED & SET \\
\hline & By Per & centage & & & By Trea & tment & \\
\hline & Untreate & & & & 10 & & \\
\hline & 10 & 20 & 30 & & Untreate & Silane & $\mathrm{DCP}$ \\
\hline Average & 66.24 & 65.75 & 61.65 & Average & 66.24 & 66.53 & 61.03 \\
\hline STDeV & 3.00 & 3.57 & 5.52 & STDeV & 3.00 & 2.55 & 12.55 \\
\hline$s 2$ & 1.7494 & 0.1599 & 3.177 & $s 2$ & 31.891 & 0.1005 & 45.596 \\
\hline Var_Ave & 6.3499 & 0.1198 & 10.528 & Var_Ave & $=9.5747$ & 0.0413 & 13.572 \\
\hline & & & & & & & 0.893 \\
\hline$F$ & 10.889 & 2.2478 & $\begin{array}{r}9.9419 \\
\text { naterial }\end{array}$ & $\begin{array}{l}F \\
f 1\end{array}$ & 0.9007 & $\begin{array}{r}1.2329 \\
\text { dof }-1 \text { in }\end{array}$ & $\begin{array}{l}0.893 \\
\text { material }\end{array}$ \\
\hline 12 & 2 & dof meas & urement & 12 & 2 & dof mea: & surement \\
\hline & & & & & & & \\
\hline & Silane & & & & 20 & & \\
\hline & 10 & 20 & 30 & & Untreate & Silane & $\mathrm{DCP}$ \\
\hline Average & 66.53 & 68.26 & 63.36 & Average & $\quad 65.75$ & 68.26 & 64.09 \\
\hline STDeV & 2.55 & 3.36 & 8.89 & STDeV & 3.57 & 3.36 & 17.83 \\
\hline$\$ 2$ & 11.884 & 0.3249 & 20.053 & $s 2$ & 68.828 & 0.0216 & 101.74 \\
\hline Var_Ave & 6.1724 & 1.496 & 5.0218 & Var_Ave & E 4 & 3.1417 & 1.3745 \\
\hline $\mathrm{F}$ & 1.5582 & 13.812 & 0.7513 & $\mathrm{~F}$ & 0.1743 & 436.85 & 0.0405 \\
\hline f1 & 1 & dof - 1 in r & naterial & f1 & 1 & dof - 1 in & material \\
\hline 12 & 2 & dof meas & urement & 12 & 2 & dof meas & surement \\
\hline & $\mathrm{DCP}$ & & & & & & \\
\hline & 10 & 20 & 30 & & Untreate & Silane & $\mathrm{DCP}$ \\
\hline Average & 61.03 & 64.09 & 66.59 & Average & 61.65 & 63.36 & 66.59 \\
\hline STDeV & 12.55 & 17.83 & 17.95 & STDeV & 5.52 & 8.89 & 17.95 \\
\hline$s 2$ & 95025 & 13943 & 14558 & 82 & 41297 & 5,6582 & 77186 \\
\hline Var_Ave & 7.7512 & 4.6893 & 15.449 & Var_Ave & $=6.2877$ & 1.4569 & 12.189 \\
\hline F & 24471 & 1009 & 31836 & F & 0.4568 & 0.7724 & 0.4737 \\
\hline f1 & & dof -1 in & naterial & f1 & & dof -1 in & material \\
\hline 12 & 2 & dof meas & urement & 12 & 2 & dof meas & surement \\
\hline
\end{tabular}

Figure A.8: F test analysis flexural strength of each treatment and fiber fraction by fiber. Green is significant with $99 \%$ confidence or greater, while red is not a statistically significant result. 


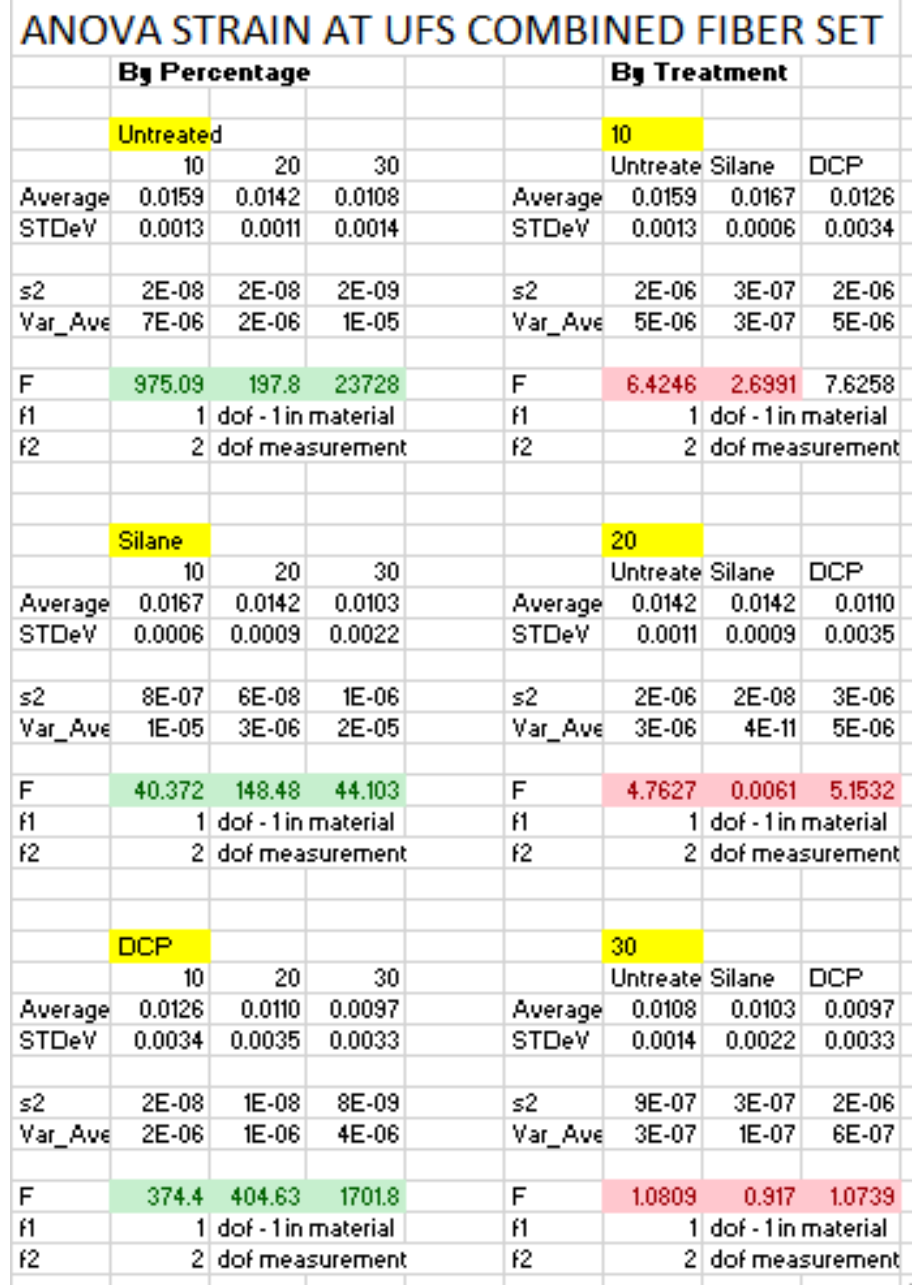

Figure A.9: F test analysis flexural strength of each treatment and fiber fraction by fiber. Green is significant with $99 \%$ confidence or greater, while red is not a statistically significant result. 
$\underline{\text { DSC Results }}$

Table A.2: Thermal properties and crystallinity of tested $20 \%$ hollow stem composites with neat and grafted PHBV for reference

\begin{tabular}{|c|c|c|c|c|c|c|c|c|}
\hline Sample & $\begin{array}{c}T_{\text {onset }} \\
\left.{ }^{\circ} \mathrm{C}\right]\end{array}$ & $\begin{array}{c}T_{m} \\
\left.{ }^{\circ} \mathrm{C}\right]\end{array}$ & $\begin{array}{r}\Delta H_{m} \\
{[\mathrm{~J} / \mathrm{g}]}\end{array}$ & $\begin{array}{c}\chi \\
{[\%]}\end{array}$ & $\begin{array}{c}T_{\text {onset }} \\
\left.{ }^{\circ} \mathrm{C}\right]\end{array}$ & $\begin{array}{c}T_{c} \\
\left.{ }^{\circ} \mathrm{C}\right]\end{array}$ & $\begin{array}{l}\Delta H_{c} \\
{[\mathrm{~J} / \mathrm{g}]}\end{array}$ & $\begin{array}{c}\Delta w^{1 / 2 p} \\
\left.\quad{ }^{\circ} \mathrm{C}\right]\end{array}$ \\
\hline PHBV & 167.32 & 174.04 & 73.25 & 50.17 & 110.76 & 102.02 & 48.57 & 9.23 \\
\hline$g \mathrm{PHBV}$ & 162.13 & 170.56 & 68.14 & 46.67 & 99.00 & 88.95 & 51.38 & 10.9 \\
\hline Unt. HS & 166.78 & 176.09 & 58.65 & 50.21 & 123.58 & 119.78 & 52.98 & 6.23 \\
\hline Sil. HS & 166.07 & 177.82 & 54.77 & 46.89 & 122.06 & 117.77 & 52.19 & 7.7 \\
\hline$g \mathrm{HS}$ (weak) & 152.71 & 152.71 & 59.99 & 51.36 & 111.82 & 104.32 & 51.5 & 8.66 \\
\hline$g \mathrm{HS}$ (strong) & 159.11 & 167.37 & 60.18 & 51.53 & 117.02 & 111.40 & 54.97 & 7.14 \\
\hline
\end{tabular}

Fiber Fraction Analysis Results

When comparing two samples at the same fiber fraction, the sample with a smaller deviation from the expected percentage was closer to the mean chord modulus only $50 \%$ of the time. In addition, higher measured fiber fraction corresponded with a higher measured density only $42 \%$ of the time. The relationship between fiber fraction and modulus was established with a high degree of confidence among all fibers and treatments, so these results are contradictory to the majority of the test data. For the purposes of this experiment, the accuracy and reliability of fiber fraction analysis was questionable enough that FFA measurements were not taken into account as part of the greater experimental conclusions. 


\begin{tabular}{|c|c|c|c|c|c|c|c|}
\hline Sample ID & Fiber & Weight (g) & $\begin{array}{l}\text { Density } \\
\left(\mathrm{g} / \mathrm{cm}^{\wedge} 3\right)\end{array}$ & $\begin{array}{l}\text { Fiber Wt. \% } \\
\text { (estimated) }\end{array}$ & $\begin{array}{l}\text { Measured } \\
\text { Fiber Fraction }\end{array}$ & $\begin{array}{l}\text { Chord Modulus } \\
\text { (Mpa) }\end{array}$ & $\begin{array}{l}\text { Average } \\
\text { Modulus }\end{array}$ \\
\hline $388-486$ & $\mathrm{HE}$ & 0.75839 & 1.256 & 10 & 15.1 & 6583 & 6500 \\
\hline $330-386$ & $\mathrm{HE}$ & 0.75128 & 1.27 & 10 & 10.7 & 6562 & 6500 \\
\hline $357-426$ & $\mathrm{HE}$ & 0.77911 & 1.278 & 20 & 26.1 & 7925 & 7750 \\
\hline $389-488$ & $\mathrm{HE}$ & 0.77169 & 1.299 & 20 & 17.1 & 7760 & 7750 \\
\hline $332-390$ & $\mathrm{HE}$ & 0.79393 & 1.299 & 30 & 30.0 & 9779 & 8400 \\
\hline $392-493$ & $\mathrm{HE}$ & 0.75259 & 1.303 & 30 & 32.3 & 8204 & 8400 \\
\hline $333-392$ & BA & 0.73278 & 1.26 & 10 & 12.8 & 5894 & 6050 \\
\hline $362-438$ & BA & 0.7497 & 1.258 & 10 & 16.5 & 6601 & 6050 \\
\hline $373-458$ & BA & 0.77277 & 1.273 & 20 & 26.0 & 7623 & 7400 \\
\hline $335-395$ & BA & 0.77222 & 1.283 & 20 & 13.3 & 7614 & 7400 \\
\hline $364-442$ & BA & 0.75065 & 1.289 & 30 & 27.7 & 7922 & 8300 \\
\hline $336-397$ & BA & 0.80042 & 1.302 & 30 & 32.4 & 8881 & 8300 \\
\hline $338-399$ & SS & 0.75234 & 1.274 & 10 & 19.3 & 6071 & 6200 \\
\hline $352-420$ & SS & 0.75435 & 1.26 & 10 & 11.5 & 6467 & 6200 \\
\hline $385-481$ & SS & 0.77149 & 1.28 & 20 & 23.7 & 7600 & 7400 \\
\hline $339-401$ & SS & 0.76643 & 1.318 & 20 & 26.0 & 7269 & 7400 \\
\hline $354-422$ & SS & 0.79758 & 1.301 & 30 & 33.5 & 9050 & 8950 \\
\hline $340-402$ & SS & 0.79253 & 1.296 & 30 & 36.3 & 8776 & 8950 \\
\hline $343-406$ & HS & 0.75405 & 1.261 & 10 & 15.9 & 6592 & 6300 \\
\hline $348-414$ & HS & 0.73412 & 1.255 & 10 & 20.1 & 6163 & 6300 \\
\hline $349-415$ & HS & 0.78065 & 1.273 & 20 & 26.6 & 7823 & 7600 \\
\hline $379-472$ & HS & 0.74745 & 1.267 & 20 & 23.9 & 7451 & 7600 \\
\hline $350-416$ & $\mathrm{HS}$ & 0.79378 & 1.298 & 30 & 30.9 & 9039 & 8600 \\
\hline $346-411$ & $\mathrm{HS}$ & 0.77442 & 1.295 & 30 & 31.9 & 8867 & 8600 \\
\hline
\end{tabular}

Figure A.10: Results of fiber fraction analysis. Two samples were tested for each combination of fiber and fraction, to see the relationship between fiber fraction and stiffness.

Supplementary Methods and Detailed Procedures

$\underline{\text { Silane Coupling Agent Selection }}$

The effectiveness of fiber treatment is highly dependent on the surface composition of the fiber and the interfacial interactions with the polymer. The silane coupling agent GPTMS has been effective at reducing fiber moisture uptake and the impact of moisture on tensile strength of natural fiber composites [57]. The structure of GPTMS is shown in Figure A.11. 


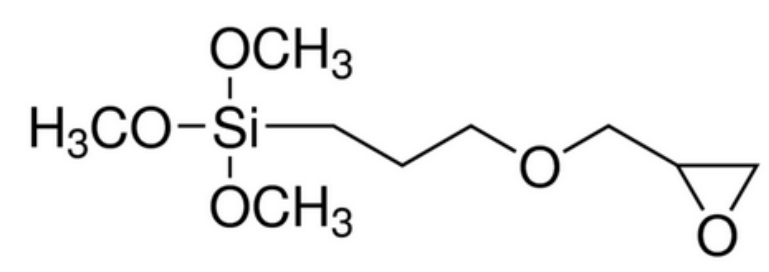

Figure A.11: Structure of GPTMS.

Silane Fiber Treatment Procedure

Equipment:

- Fume hood

- Plastic dome desiccator

- Vacuum pump (with all hoses and connections for desiccator)

- Eye protection

- Gloves

- Aluminum foil

- Parafilm

- Small size weighing dish

- Syringe (3mm)

- Nitrogen gas cylinder

- Silanating agent: (3-Glycidyloxypropyl)trimethoxysilane (GPTMS)

- Predried natural fiber

Physical setup: 
1. Turn fume hood on

2. Place desiccator and pump inside hood

3. Connect desiccator to pump, ensure good seal on all tubing components

4. With a large sheet of aluminum foil, create a "cup" configuration with fiber

5. Place pocket on bottom of desiccator, as flat as possible (See Figure A.12).

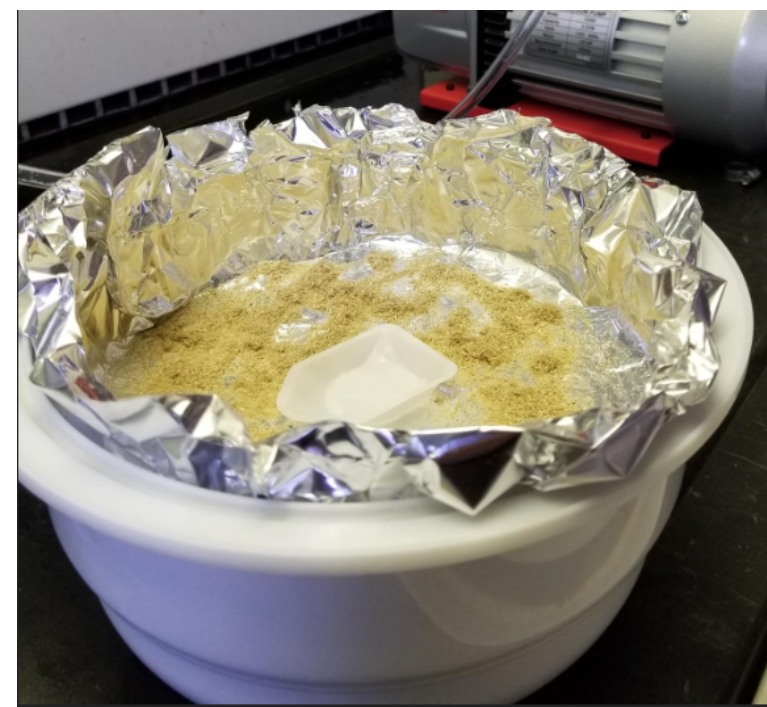

Figure A.12: Cup configuration with natural fibers and silane droplet on dish in center.

\section{Room temperature vapor deposition:}

1. Put on eye protection and gloves

2. With a large sheet of aluminum foil, create a "cup" configuration with fiber spread evenly

3. Place pocket on bottom of desiccator, as flat as possible (See Figure A.12). 
4. Take weighing dish and place in center of cup, ensuring it does not cover any fiber

5. Using syringe, place 1 small drop ( $.1 \mathrm{~mL}$ ) GPTMS on center of dish

6. Fold edges of cup to close completely

7. Put desiccator lid over cup

8. Turn desiccator valve to closed position

9. Turn on vacuum pump

10. Slowly open desiccator valve to open position

11. Degas for 3.5 minutes.

12. Close valve, shut off pump, and lower hood sash

13. Wait 1 hour

14. Raise hood sash

15. Turn pump on

16. Slowly open desiccator valve to open position

17. Degas for 3.5 minutes

18. Close valve, shut off pump, and lower hood sash

19. Wait 1 hour

20. Raise hood sash

21. Open vacuum seal on pump 
22. Slowly open desiccator valve to open position

23. Allow normal air pressure to return to desiccator

24. Remove desiccator lid

25. Remove pocket from desiccator (still within hood)

26. Remove treated fibers

PHBV Grafting Procedure

Equipment

- Fume hood

- Stir Plate with Rod

- Beakers, >100 ml volume

- Eye protection

- Gloves

- Aluminum foil

- Powdered PHBV

- Acetone

- Predried natural fiber

\section{Chemical procedure}

1. Put on eye protection and gloves.

2. Turn fume hood on. 
3. For powder matrix:

(a) Place beaker with $100 \mathrm{ml}$ acetone on stir plate

(b) Place stir bar inside beaker

(c) Turn stirrer to low speed (setting 2)

(d) Add DCP to acetone, let dissolve

(e) Add matrix to acetone

4. For fiber:

(a) Place beaker with $50 \mathrm{ml}$ acetone on stir plate

(b) Place stir bar inside beaker

(c) Turn stirrer to low speed setting

(d) Add DCP to acetone, let dissolve

(e) Add fiber to acetone

5. For both powder matrix and fiber, allow 30 minutes for coating.

6. Pour mixture into aluminum foil "cup" and let dry

7. Mix fiber and polymer in beaker immediately before injection molding.

\section{Supplemental Composite Fabrication}

A flat leveling of the torque/pressure curve was an indication of even mixing throughout the material, as any areas with clumps of material or uneven viscosity would cause a visual spike on the feed (see Figure A.13).

Compounding and molding parameters were determined through repeated iterations of all fibers at a 10\% and $30 \%$ fraction, to determine what temperature and 


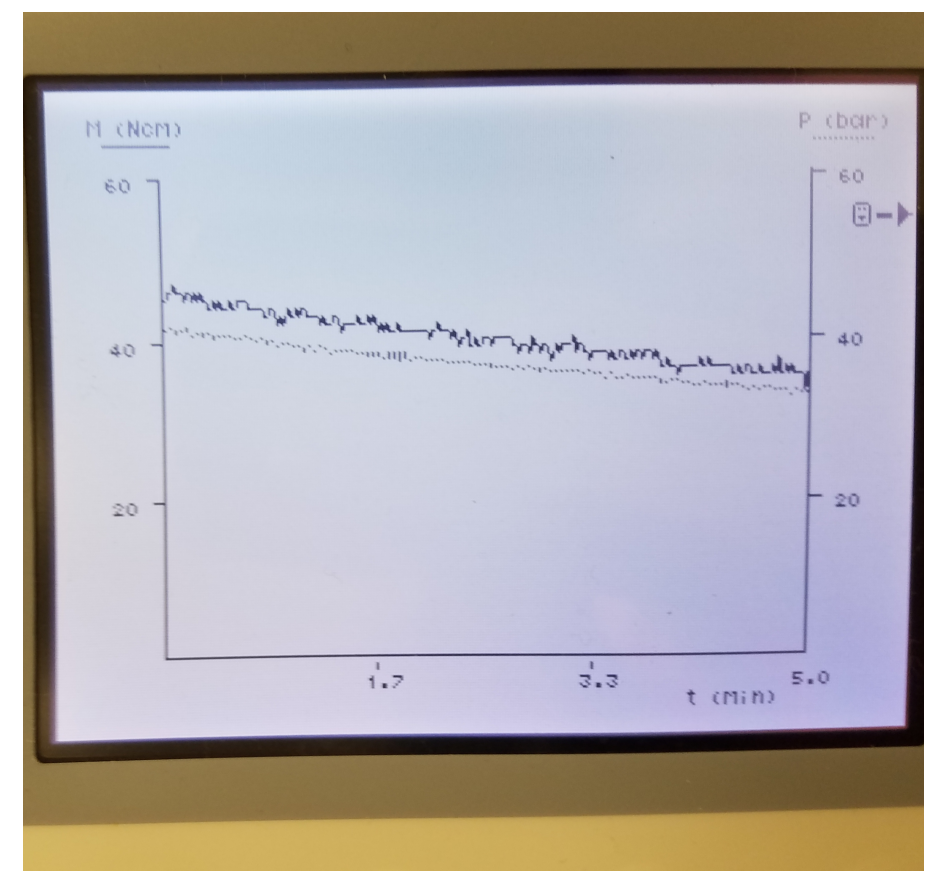

Figure A.13: Steady leveling curve was used as an indicator of even mixing.

speed values would successfully generate samples without jamming in the extrusion process, partial fills in the injection process, or pitting on the samples. To rapidly solidify the sample and keep fiber from accumulating at the bottom of the mold, mold temperature was reduced to $50^{\circ} \mathrm{C}$. Higher mold temperatures were found to have a less even dispersion of the fiber across the sample (as seen on the surface). Lower mold temperatures would cause premature solidification and partial fills of the mold.

For AF- $g$-PHBV grafted samples, uneven mixing in the treatment process may cause local increases in DCP concentration, and any time variations in the wet mixing, dry mixing, feeding, or compounding processes could have an impact on the net reaction time and the physical properties. These parameters must be monitored closely during the treatment process to ensure a successful graft. 
Fiber Fraction Analysis Procedure

\section{Equipment}

- Biocomposite sample (.77 grams)

- Weigh boat

- Density kit

- Scale

- Gloves

- Safety Glasses

- Hood

- Hot/stir plate

- $500 \mathrm{ml}$ beaker

- Glass funnel

- Condensation Tower

- Vacuum grease

- Stir bar

- Chloroform

- Ceramic funnel

- 20 um paper filter

- Rubber stopper 
- Beaker with hose connection

- Vacuum with hose

- Spatula

\section{Weighing/Density}

1. Weigh sample and calculate density using /hlscale process

2. log weight of weigh boat

\section{Dissolution}

1. clean all glassware before use

2. Set hot plate to Heat 4

3. place sample and stir bar in beaker

4. add $50 \mathrm{ml}$ of chloroform to beaker through glass funnel

5. put grease on bottom of condensation tower and place over beaker, turning slowly to spread grease

6. place beaker+tower on hot plate and set Stir to 7

7. Record time at start

\section{Filtration}

1. Place rubber stopper between hose beaker and ceramic funnel (this is not the dissolution system!)

2. Connect vacuum hose to hose beaker 
3. Place filter in funnel, wet with chloroform to anchor

4. Remove beaker/tower setup from hot plate, record time

5. turn on vacuum

6. remove tower, remove stir bar

7. slowly pour out solution from tower into ceramic funnel

8. use chloroform to rinse out remaining fiber from beaker into filter

9. allow filter/fibers to dry (one procedure says to allow a week for this?)

10. empty all fibers into weigh boat, use spatula to scrape any remaining fiber

11. dispose of plastic waste in hazard waste container

12. Weigh all fibers and subtract weigh boat 
$\underline{\text { APPENDIX B }}$

MATLAB CODE 
flexdataloader.m

for $n 0=1: 1$

clc

close all

clear

prompt='What is $_{\sqcup}$ the specimen $_{\sqcup}$ batch $_{\sqcup} I D ?_{\sqcup}:^{\prime}$ ';

batchid=input (prompt, 's') ;

$\operatorname{int} \mathrm{YN}=1$;

if int $\mathrm{YN}==1$

oldFolder $=\mathbf{c d}$;

filex='.csv';

cd ..\TensileTestRawData

RawData= $\operatorname{csvread}($ strcat (batchid,filex), 11,0$)$;

cd(oldFolder)

end

\% The Data Column Labels for RawData are as follows \%

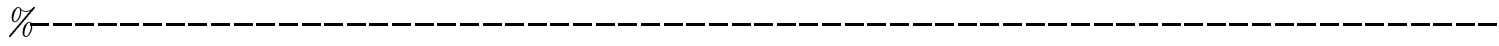

\% 1. Time

\%2. Extension

\%3. Load

\%4. Flexure Extension

\%5. Flexure Load 


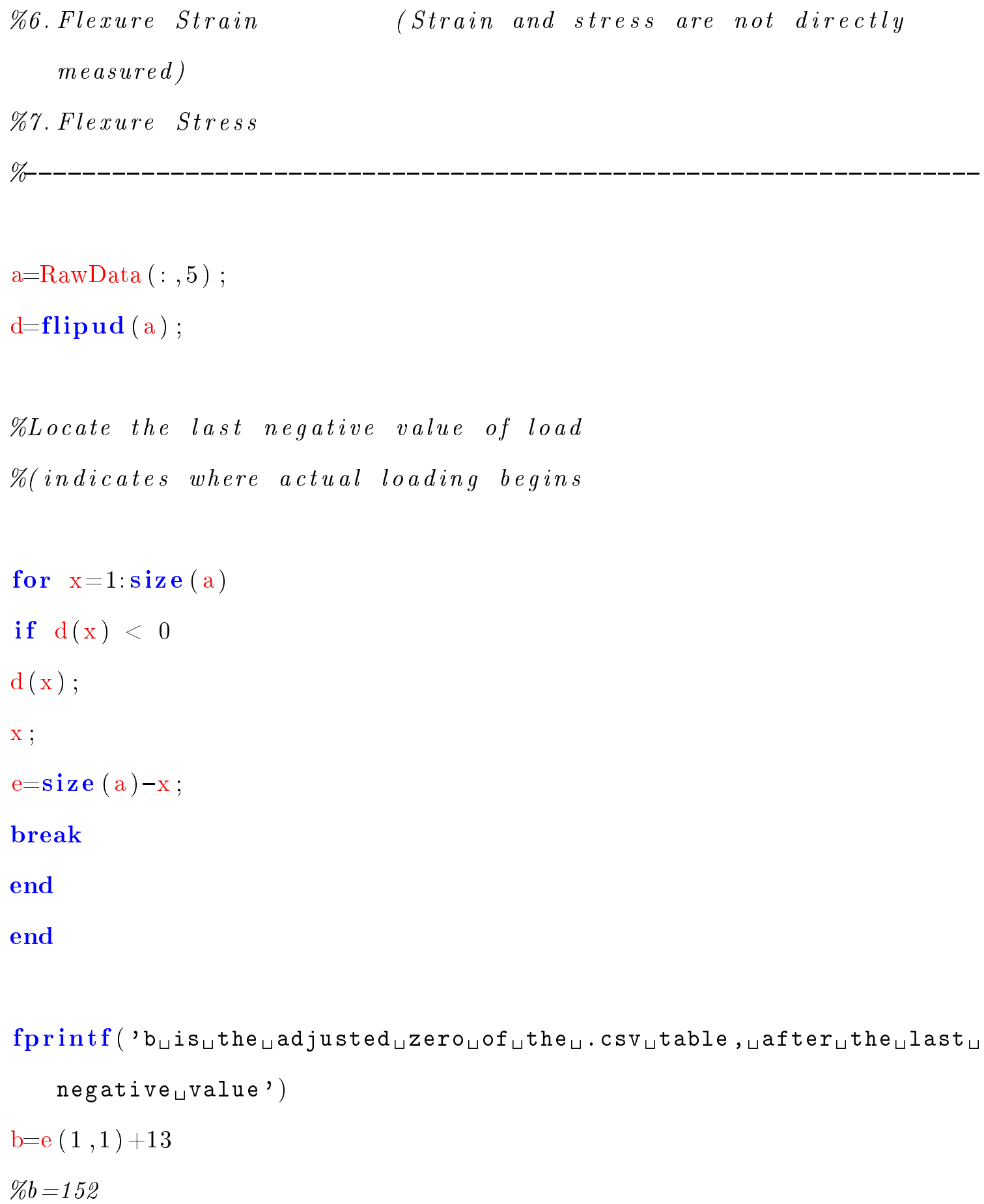


adj=RawData $(b-11,4)$;

$\operatorname{RawData2}(:, 4)=\operatorname{RawData2}(:, 4)-\operatorname{adj} ;$

\%The following columns are set to 0 because

\%they are meaningless after the zero shift

$\operatorname{RawData2}(:, 2)=0$;

$\operatorname{RawData2}(:, 3)=0$;

$\mathrm{x}=\operatorname{RawData} 2(:, 4)$;

$\mathrm{y}=\operatorname{RawData} 2(:, 5)$;

\section{figure (1)}

hold on

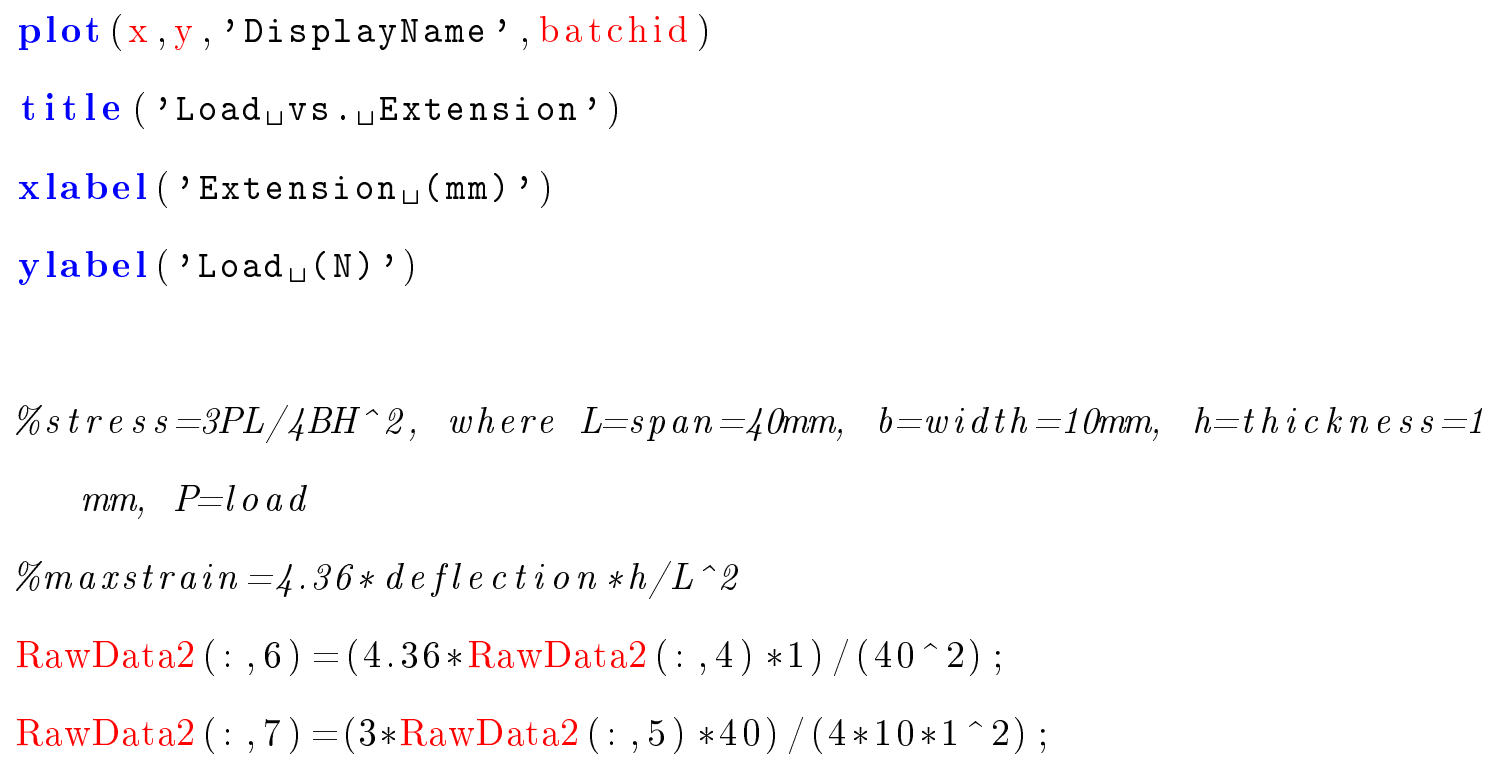


Strain---Stress ')

RawData2;

\%fprintf('----Time---------empty-----empty-Extension---Load----Strain---Stress ')

Load=RawData2 $(:, 5)$;

$\mathrm{cc}=\max (\operatorname{RawData2}(:, 5))$;

for $\mathrm{x} 1=1: \mathrm{size}(\mathrm{Load})$

if $\operatorname{Load}(\mathrm{x} 1)=\mathrm{cc}$

MaxLoadN=Load (x1)

MaxExtensionMM=RawData2 $(x 1,4)$

MaxStressMPA=RawData2 (x1, 7)

MaxStrain=RawData2 $(x 1,6)$

break

end

end

Strain $=\operatorname{RawData2}(:, 6)$;

for $x 2=1:$ size (Strain)

if $\operatorname{Strain}(\mathrm{x} 2)>=0.001$

strain 11 o $c=x 2$;

break

end

end 


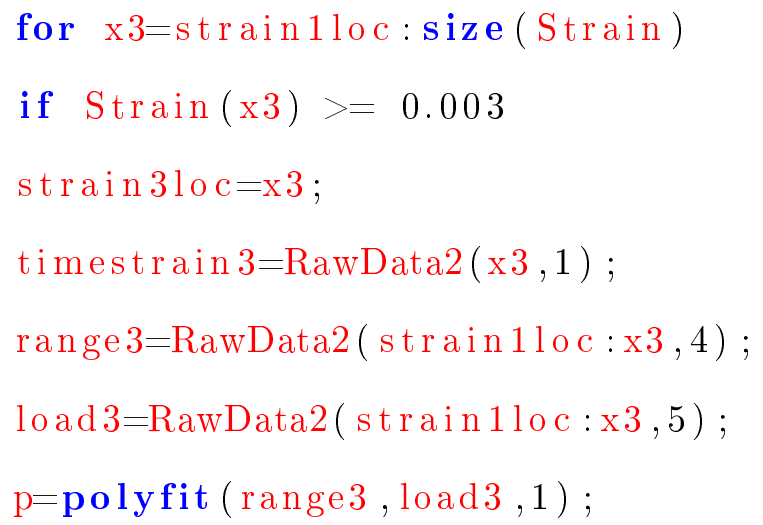




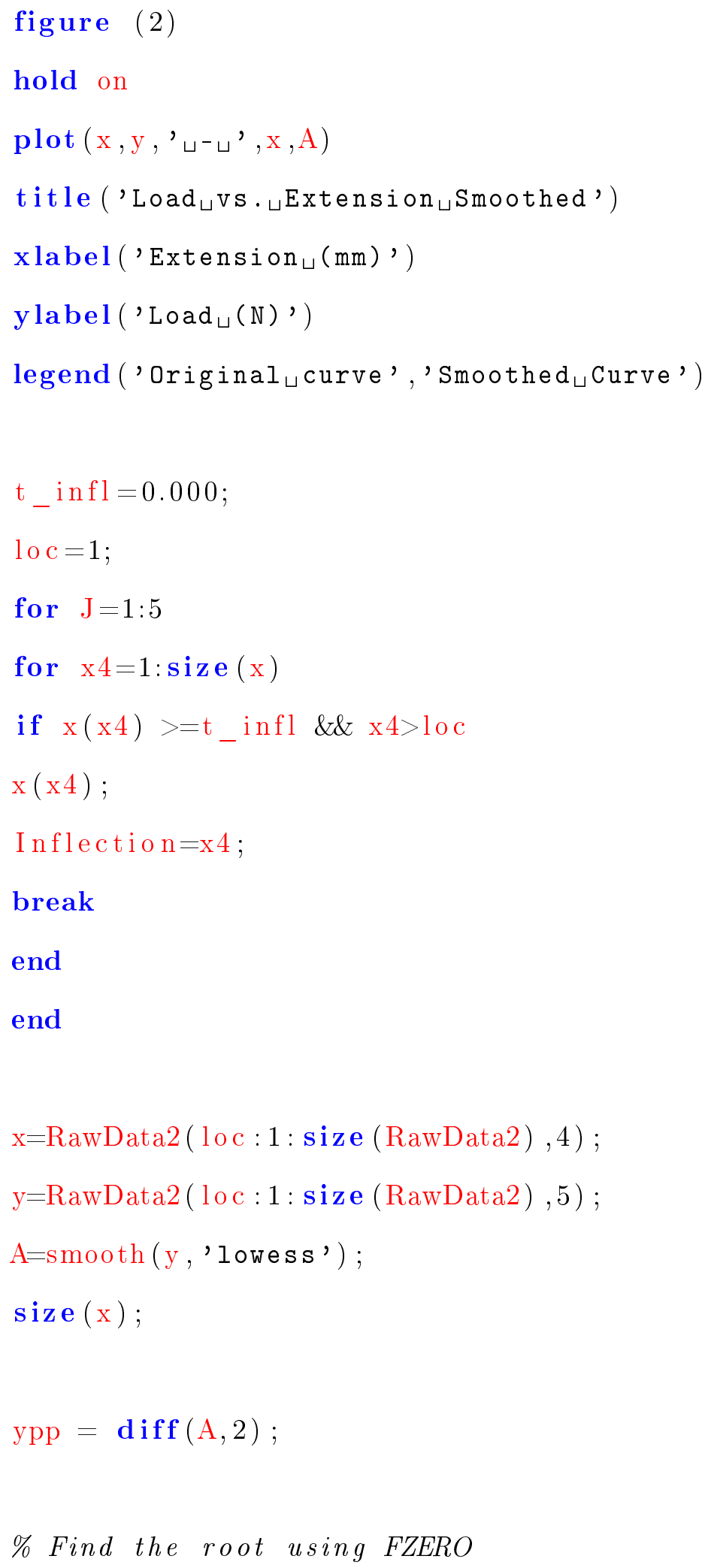




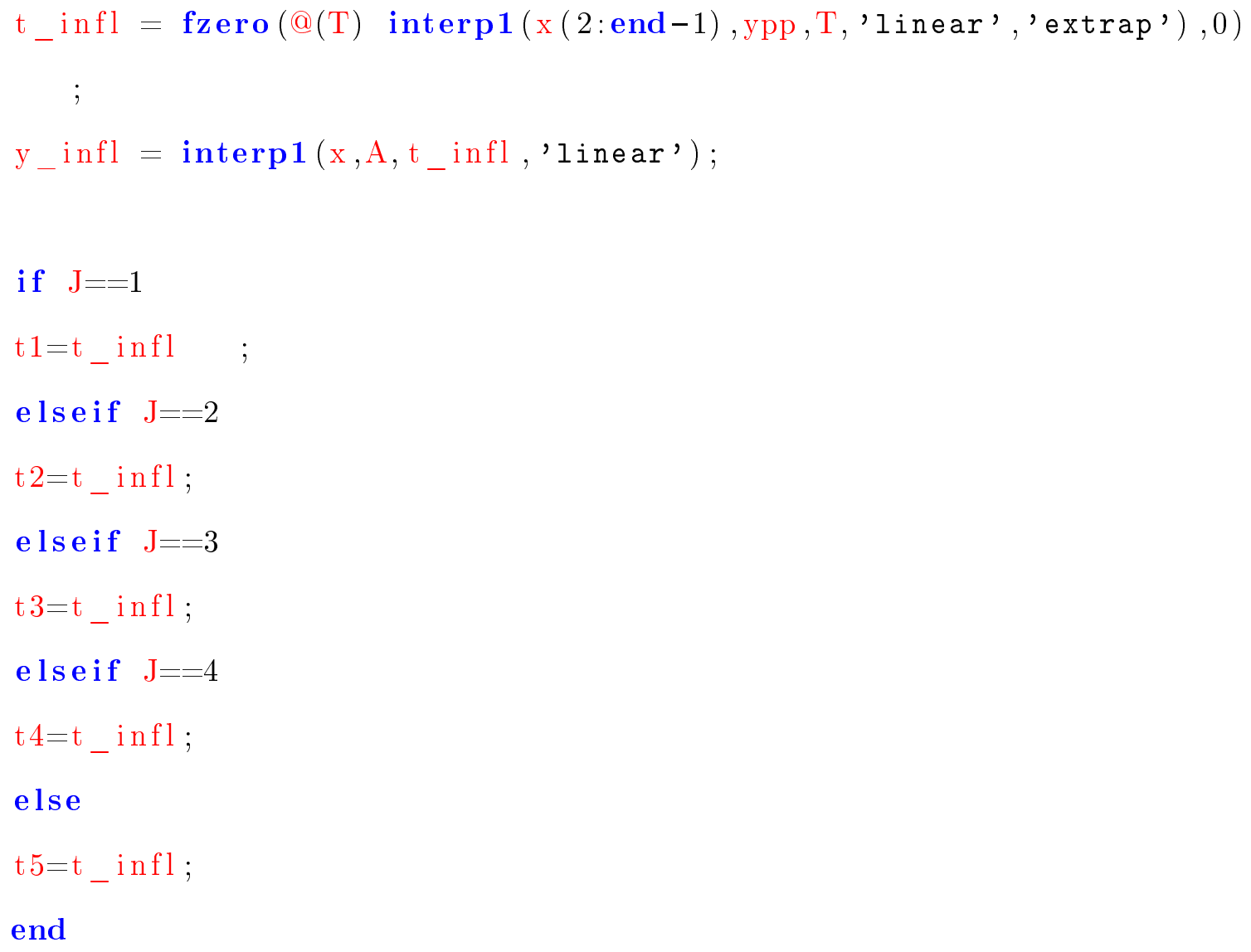




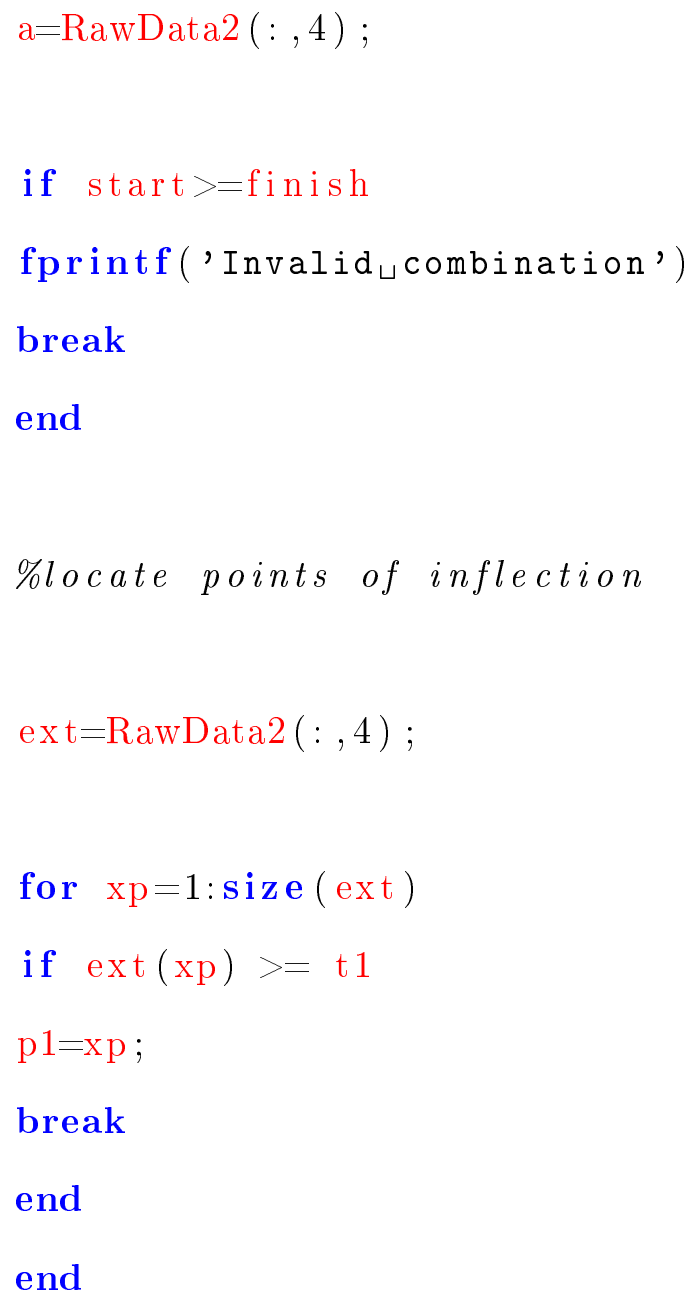




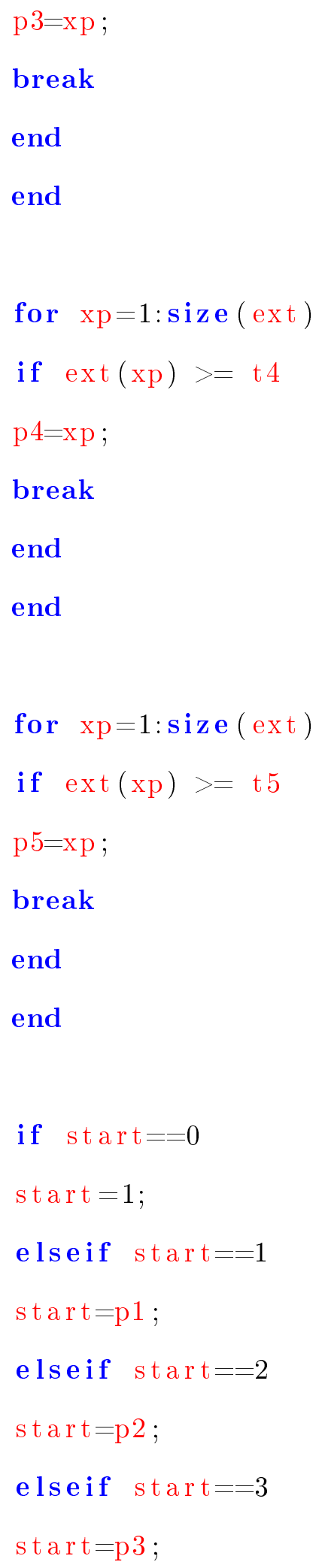




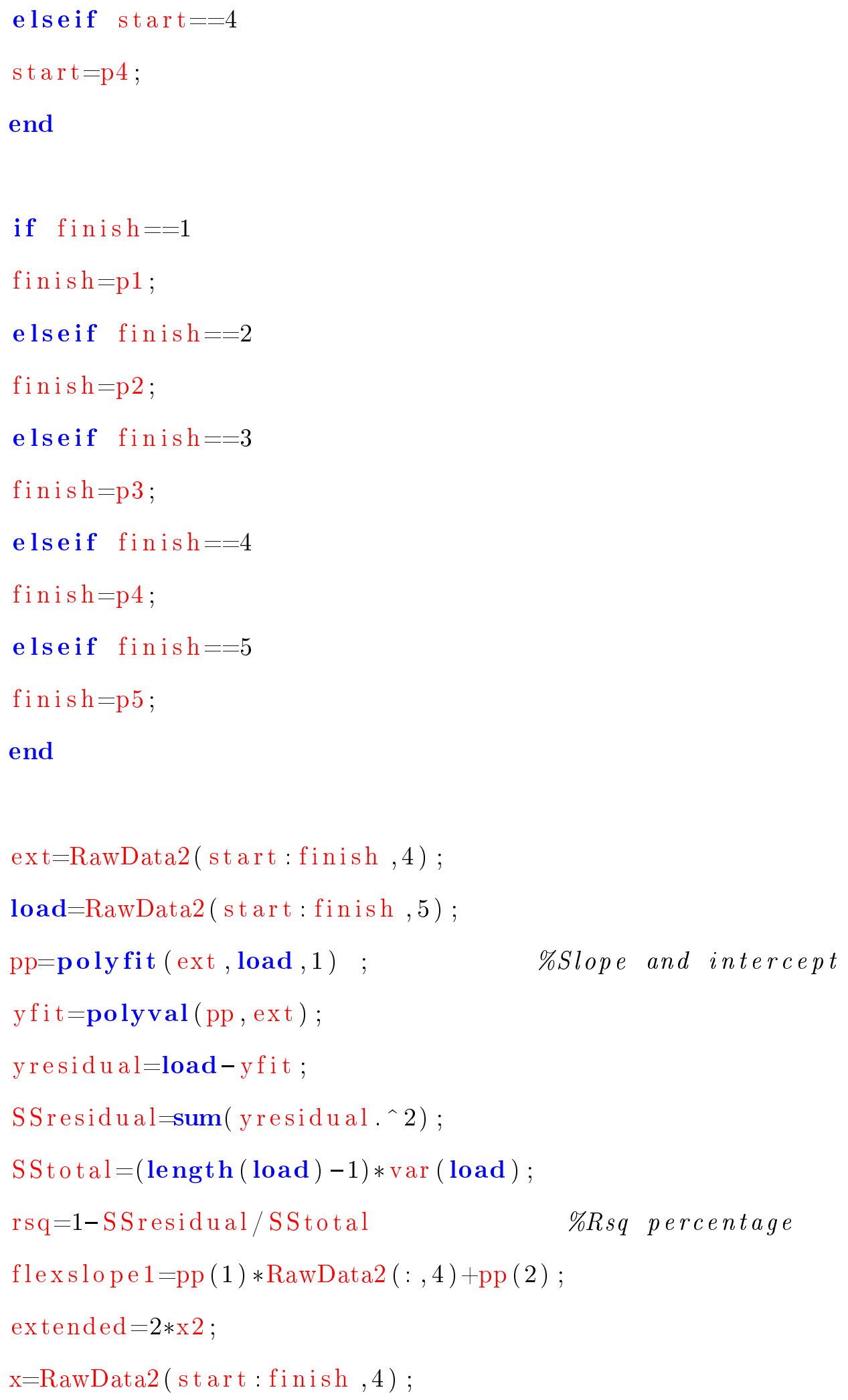


$\mathrm{y}=$ RawData2( start:finish , 5);

SecantModulus $=.17 * 40^{\wedge} 3 * \operatorname{pp}(1) /\left(10 * 1^{\wedge} 3\right)$

$\operatorname{plot}(x, y$, 'g')

end 